\title{
Design of Fire Detection Algorithm Based on Fuzzy Logic and using Wireless Sensors
}

\author{
by \\ Xiao Jie Chu \\ A thesis submitted to the Faculty of Graduate and Postdoctoral Affairs in partial \\ fulfillment of the requirements for the degree of \\ Master of Applied Science in Electrical and Computer Engineering \\ Ottawa-Carleton Institute of Electrical and Computer Engineering (OCIECE) \\ Department of System and Computer Engineering \\ Carleton University \\ Ottawa, Ontario, Canada, K1S 5B6
}

December 2010

(C) Copyright 2010, Xiao Jie Chu 


$\begin{array}{ll}\begin{array}{l}\text { Library and Archives } \\ \text { Canada }\end{array} & \begin{array}{l}\text { Bibliothèue et } \\ \text { Archives Canada }\end{array} \\ \begin{array}{l}\text { Published Heritage } \\ \text { Branch }\end{array} & \begin{array}{l}\text { Direction du } \\ \text { Patrimoine de l'édition }\end{array} \\ \begin{array}{l}\text { 395 Wellington Street } \\ \text { Ottawa ON K1A ON4 } \\ \text { Canada }\end{array} & \begin{array}{l}\text { 395, rue Wellington } \\ \text { Ottawa ON K1A ON4 } \\ \text { Canada }\end{array}\end{array}$

Your file Votre référence

ISBN: 978-0-494-81712-4

Our file Notre référence

ISBN: 978-0-494-81712-4

NOTICE:

The author has granted a nonexclusive license allowing Library and Archives Canada to reproduce, publish, archive, preserve, conserve, communicate to the public by telecommunication or on the Internet, loan, distribute and sell theses worldwide, for commercial or noncommercial purposes, in microform, paper, electronic and/or any other formats.

The author retains copyright ownership and moral rights in this thesis. Neither the thesis nor substantial extracts from it may be printed or otherwise reproduced without the author's permission.
AVIS:

L'auteur a accordé une licence non exclusive permettant à la Bibliothèque et Archives Canada de reproduire, publier, archiver, sauvegarder, conserver, transmettre au public par télécommunication ou par l'Internet, prêter, distribuer et vendre des thèses partout dans le monde, à des fins commerciales ou autres, sur support microforme, papier, électronique et/ou autres formats.

L'auteur conserve la propriété du droit d'auteur et des droits moraux qui protège cette thèse. $\mathrm{Ni}$ la thèse ni des extraits substantiels de celle-ci ne doivent être imprimés ou autrement reproduits sans son autorisation.
In compliance with the Canadian Privacy Act some supporting forms may have been removed from this thesis.

While these forms may be included in the document page count, their removal does not represent any loss of content from the thesis.
Conformément à la loi canadienne sur la protection de la vie privée, quelques formulaires secondaires ont été enlevés de cette thèse.

Bien que ces formulaires aient inclus dans la pagination, il n'y aura aucun contenu manquant. 
The undersigned recommend to

the Faculty of Graduate Studies and Postdoctoral Affairs acceptance of the thesis

\title{
Design of Fire Detection Algorithm Based on Fuzzy Logic and using
} Wireless Sensors

\author{
submitted by \\ Xiao Jie Chu
}

In partial fulfillment of the requirements for the degree of Master of Applied Science in Electrical and Computer Engineering

\begin{tabular}{c} 
Professor Howard M. Schwartz \\
Chair, Department of Systems and Computer Engineering \\
\hline Professor Jérôme Talim \\
Thesis Supervisor \\
Carleton University \\
December 2010
\end{tabular}




\section{Abstract}

Wireless Sensor Networks are popularly used in monitoring systems, especially in fire detection systems. Accurately detecting fires and efficiently immunizing against false cases are the key objectives researchers are trying to achieve.

Our fire detection algorithms use simple temperature sensors to explore temperature-developing trends, combining neighborhood observation using Fuzzy Logic System. Furthermore, a robust fire estimation algorithm is proposed with local and global level data processing at sensor and Base Station respectively to estimate the direction of disaster.

The performance of the fire detection algorithm proves that the use of simple temperature sensors with the assistance of data fusion technology is able to detect fires successfully. The algorithm is validated by both fire alarm cases and nuisance cases. Upon fire detection, the Base Station estimates the fire-spreading trajectory using the proposed propagation algorithms. 


\section{Acknowledgments}

I would like to express my deepest and most sincere gratitude to my supervisor, Professor Jérôme Talim for his guidance during my research and study at Carleton University. His immense knowledge, continuous support, inspiration, kindness and patience helped me in all the time of research and writing of this thesis. This work would not have been possible without his great efforts.

I am thankful to the defence committee members, Professor Yvan Labiche, Professor F. Richard Yu, and Professor Oliver W,W, Yang for their valuable comments and feedback. Their constructive criticisms not only helped me to improve the quality of my thesis but also encouraged me to push my limits.

Lastly, I would like to thank my husband Huicheng Guo and my son Xiangshen Guo for all their understanding and constant support in my research. 


\section{Table of Contents}

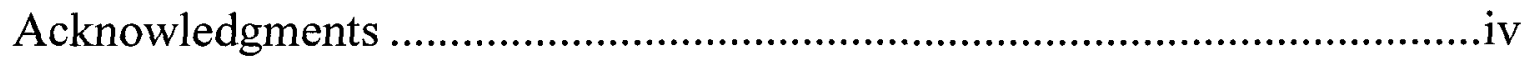

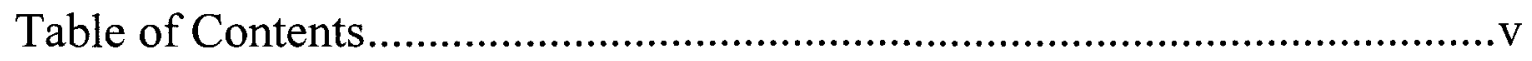

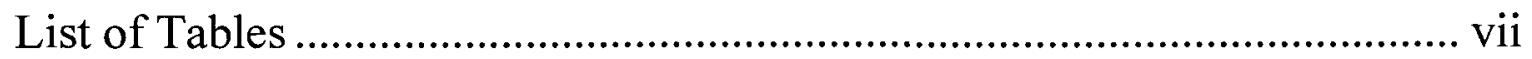

List of Figures .....................................................................................

Chapter 1 - Introduction .....................................................................

Chapter 2 - Literature Review ................................................................ 4

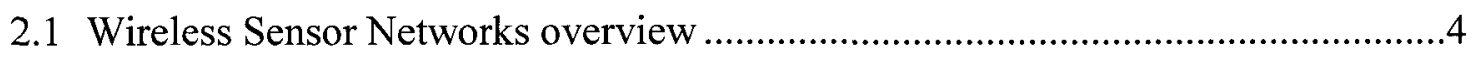

2.2 Fire Detection Techniques Overview ................................................................6

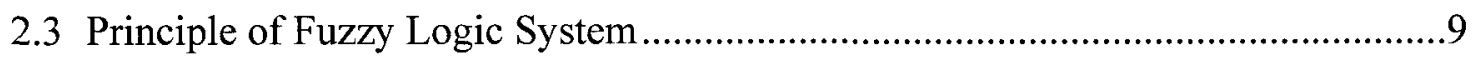

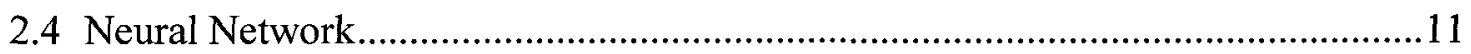

2.5 Applications of Fuzzy Logic System in the Wireless Sensor Networks ................13

2.6 Using Wireless Sensor Networks for the Estimation of Fire Propagation

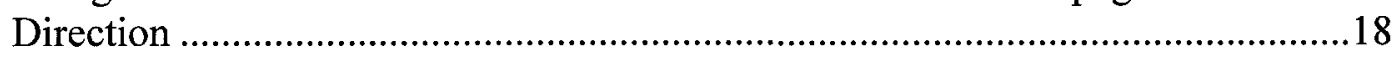

2.7 Implementation of Fire Detection Algorithm using Fuzzy Logic System..............21

2.8 Implementation of Direction Estimation Algorithm using Wireless Sensor

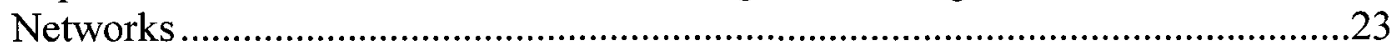

Chapter 3 - Fire Detection Algorithms using Fuzzy Logic System ..............25

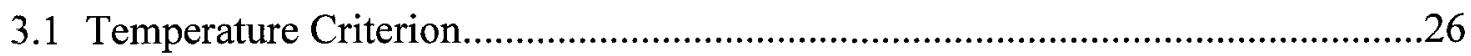

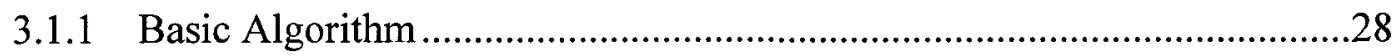

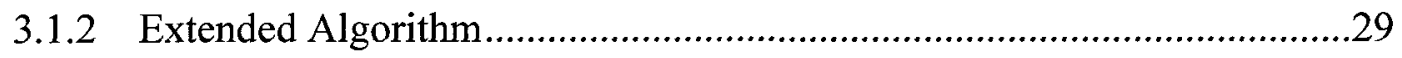

3.2 Neighborhood Agreement with Fuzzy Logic System..............................................30

3.2.1 Neighborhood Agreement Algorithm ..........................................................30

3.2.2 Using Fuzzy Logic Inference System to Make Decision.............................32

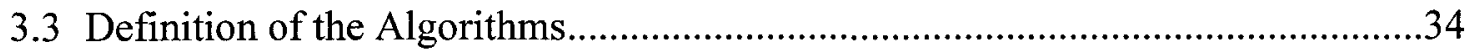




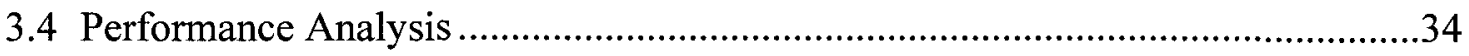

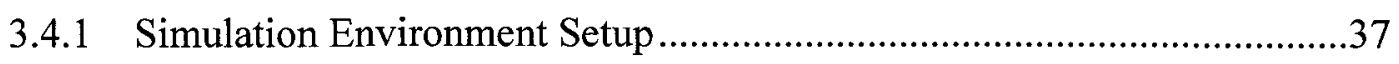

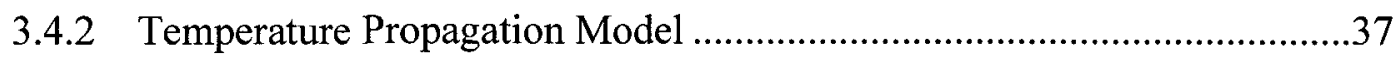

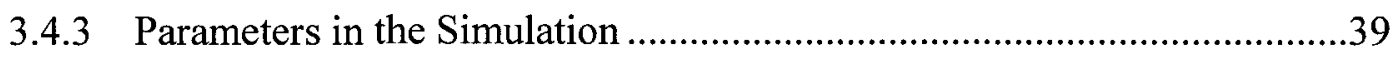

3.4.4 Simulation Scenarios ........................................................................

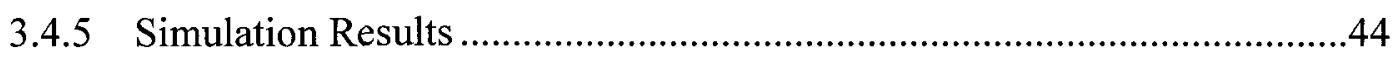

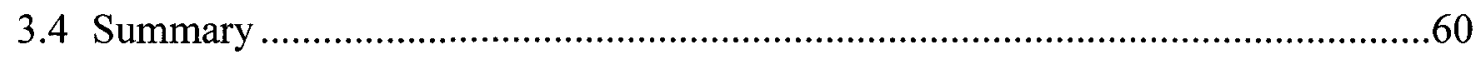

Chapter 4 - Fire Propagation Estimation ..................................................62

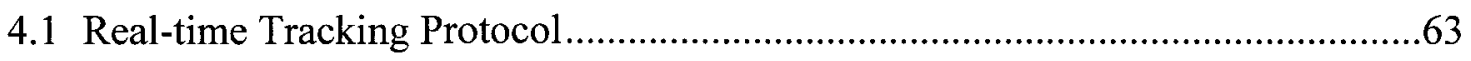

4.1.1 Local Periodic Tracking............................................................................63

4.1.2 Base Station (BS) Processing the Location Messages .................................67

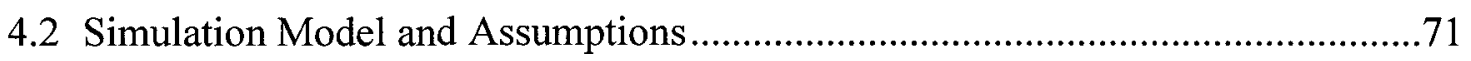

4.2.1 Disaster Propagation Model.................................................................72

4.2.2 Parameters in the Simulation ................................................................

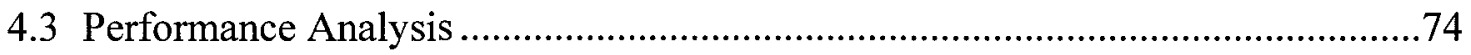

4.3.1 Example of BS Determining Fire Direction ...............................................74

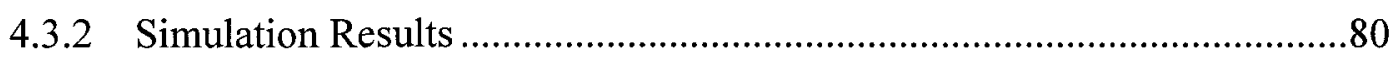

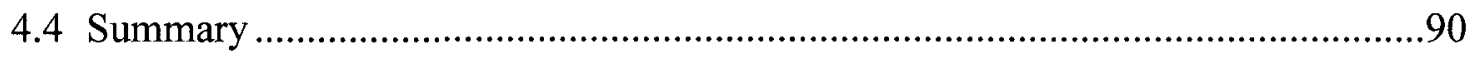

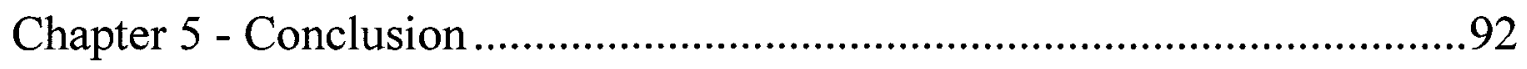

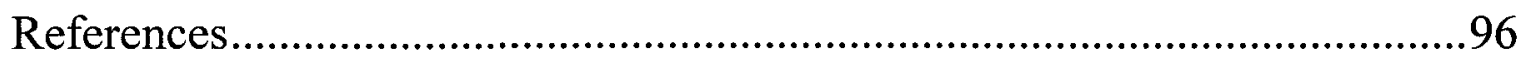




\section{List of Tables}

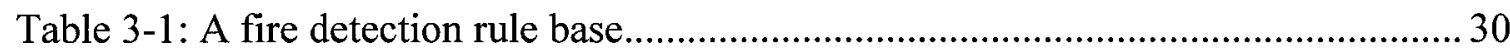

Table 3-2: Sample of the temperature data ......................................................................... 39

Table 3-3: Parameters used in the simulation program ……………………………....... 40

Table 3-4: The results of SDC 10 with Fire Detection Algorithm 1 tperiod from $1 \mathrm{~s}$ to $5 \mathrm{~s}$ 45

Table 3-5: The results of SDC10 with Fire Detection Algorithm 1 tperiod from 6s to 16s

Table 3-6: The results of SDC10 with Fire Detection Algorithm 1 tperod from 17s to 20s

Table 3-7: The results of SDC36 with Fire Detection Algorithm 1 tperiod from 1s to $5 \mathrm{~s} .48$

Table 3-8: The results of SDC36 with Fire Detection Algorithm 1 tperiod from 6 to $16 \mathrm{~s} .48$ Table 3-9: The results of SDC36 with Fire Detection Algorithm 1 tperiod from 17s to 20s

Table 3-10: The results of MNH06 with Algorithm 1 ...................................................5

Table 3-11: The results of MNH33 with Algorithm 1 ................................................... 51

Table 3-12: The results of SDC10 with Algorithm 2 ...................................................52

Table 3-13: The results of MNH33 with Algorithm 2 …................................................ 53

Table 3-14: Fire alarm time of SDC 10 of Algorithm1 and Algorithm2 ......................... 54

Table 3-15: Real fire SDC10 with and without neighborhood agreement ......................55

Table 3-16: False alarm case MNH33 with and without neighborhood agreement .........55

Table 3-17: The alarm time of real fire scenario SDC10 with different LB and UB ...... 59

Table 3-18: The alarm time of false alarm scenario MNH33 with different LB and UB 60

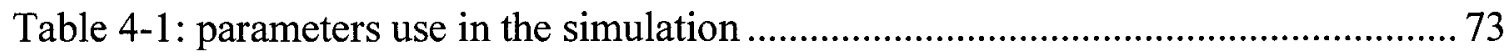

Table 4-2: Center of gravity points BS received in the first $\mathrm{W}=30$ seconds .................... 75

Table 4-3: Center gravity points BS received in the second $\mathrm{W}=30$ seconds.................... 77 
Table 4-4: The summary of the results

Table 4-5: Number of vectors and the percentages in each section................................. 80

Table 4-6: The results of the 3 scenarios with simple algorithm..................................... 81

Table 4-7: The results of 3 scenarios with static algorithm ............................................ 82

Table 4-8: The results of 3 scenarios with statistical algorithm with maximum number of

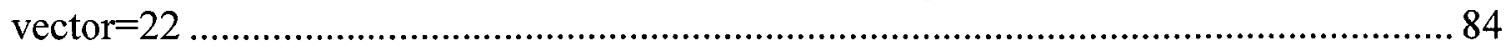

Table 4-9: Simulation results respects to the sensor density .............................................8 89 


\section{List of Figures}

Figure 2-1: Wireless Sensor Network [4] ...............................................................

Figure 2-2: The structure of fuzzy logic system [19] …................................................ 10

Figure 2-3: Architecture of a Neural Network................................................................ 12

Figure 2-4: An FFNN with 3 neurons in input layer, 2 neurons in hidden layer and 1 neuron in output layer along with their weights ............................................................ 13

Figure 3-1: The procedure of spatial approach algorithm ................................................31

Figure 3-2: Spatial approach membership function............................................................... 33

Figure 3-3: Temperature in living room, $20 \mathrm{~mm}$ from the ceiling...................................36

Figure 3-4: Temperature in living room, $300 \mathrm{~mm}$ from the ceiling...............................36

Figure 3-5: Simulation grid with the example cell $(2,3)$ and some sensor nodes with the communication radius $r$. Base Station is at $(100,100)$................................................. 38

Figure 3-6: Temperature data of living room flaming chair SDC10 from 0 seconds to

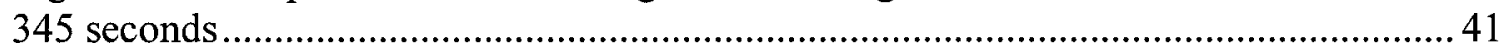

Figure 3-7: Temperature data of living room flaming chair SDC10 from 55 seconds to

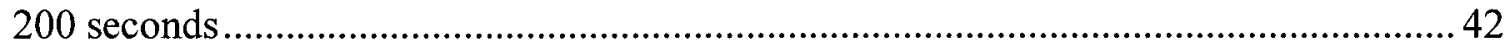

Figure 3-8: The temperature data of bedroom flaming mattress SDC36 ........................ 42

Figure 3-9: The temperature data of toasting frozen bagel MNH0 ................................. 43

Figure 3-10: The temperature data of broiling frozen pizza MNH33 ............................. 43

Figure 3-11: The temperature data of MNH33 in 1-30 seconds (left) and 290-320

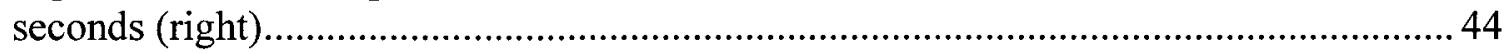

Figure 3-12: The fire detection successful rate of the 2 real fire scenarios with Algorithm 1

Figure 3-13: False alarm rate of MNH33 comparison between Algorithm1 and Algorithm2

Figure 3-14: The false alarm rate of the Algorithm 1 and 2 with or without Neighborhood Checking

Figure 3-15: Left: the relation between $\mathrm{x}$ and UB, Right: the relation between $\mu(\mathrm{x})$ and UB . 
Figure 3-16: Left: the relation between $x$ and LB, Right the relation between $\mu(x)$ and LB

Figure 4-1: Local Section of the node A and node B ....................................................... 63

Figure 4-2: Reformed local section of the node for calculation of center of gravity point 66

Figure 4-3 Fire spreading model example 72

Figure 4-4: $200 \times 200$ grid with the fire starts at $(150,150)$ and spreads towards South-

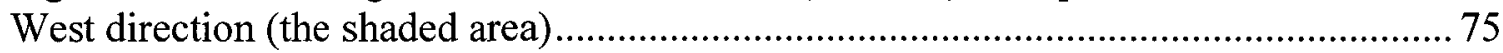

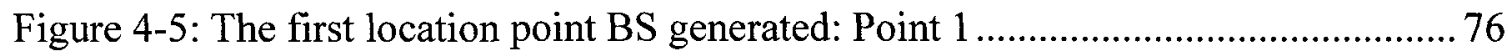

Figure 4-6: The $1^{\text {st }}$ and $2^{\text {nd }}$ location points: point 1 and point 2, the first vector............. 77

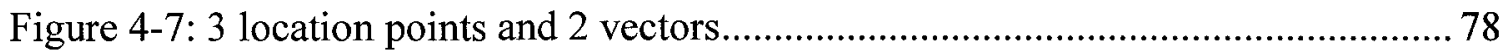

Figure 4-8: BS generates 11 points and 10 vectors....................................................... 79

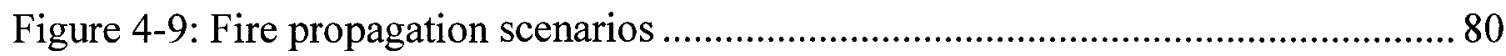

Figure 4-10: Comparison the successful rate with the different algorithm .....................83

Figure 4-11: Comparison of the successful rate with the different maximum number of vector collected using statistic algorithm......................................................................... 86

Figure 4-12: 2 irregular sensor distribution models and 1 regular distribution model ....88

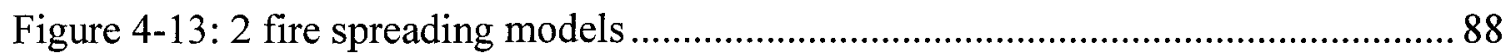

Figure 4-14: Comparison of impact of sensor density ...................................................... 90 


\section{Chapter 1 - Introduction}

In the rapid development of sensor technology, the sensor nodes are becoming simpler and less expensive. With the capability of monitoring physical or environmental conditions, such as temperature, humidity, sound, vibration, pressure etc, sensor applications are gradually pervading into many industrial and civilian application areas. These area include industrial process monitoring and control, machine health monitoring, environment and habitat monitoring, health care application, home automation and traffic control [9]. The important features of the sensors are easy installation, self-identification, self-diagnosis, reliability, time awareness for coordination with other nodes, software functions and DSP, standard control protocols and network interfaces [4]. Wireless Sense Network (WSN) consists of large amount of low cost, small size, low-power nodes, which is integrated with limited computation, sensing, and equipped with radio communication capability and sinks [22] [25], providing the flexible intelligent environment that wires or cabling can not implement.

Within the numerous WSN applications, one of the most popular applications is monitoring systems especially disaster detection system e.g. fire detection system. As everyone is aware of the destructivity of the fire, no matter in residences or in wild areas, 
early detection with accurate location and spreading directions help fire fighters react immediately and keep the losses to the minimum. The application of sensor nodes to monitoring systems requires optimizing data collection and data transportation from sensor nodes to Base Station. Many researches are investigating the sensors combination and appropriate data processing techniques for early fire detection and intelligent routing for the data transport. However only few studies are related to estimate the disaster propagation direction.

In the thesis, we focus on the processing of the data collected by the sensor nodes to detect fires. Sensors are capable of collecting temperature, $\mathrm{CO}$ density, smoke, light etc depending on the sensors equipped. Some researches for instance [14] present Fuzzy Logic approach combining the temperature and smoke density to detect fires. Our research exploits a temperature sensor, which is one of the cheapest sensors [2], to collect the temperature from environment. The sensor nodes implement the thresholds to launch the comparison between temperature changes which includes the most recent 2 collections and also extends to the previous 5 collections and the thresholds to determine the trends of the temperature development. In addition the sensor node takes the neighbors' observation into account by using Fuzzy Logic System to decide probability of the fire.

Followed by the early detection, human intervention is required to eliminate the disaster. The estimation of the fire trajectory tremendously helps the firefighters in their task. We propose an innovated algorithm to effectively estimate the development of the disaster involving local sensor tracking at the sensor and globally data processing at Base Station. Locally a sensor node is periodically checking neighbors' status so that the node 
would quickly detect if any disaster happens in its local area. The node then calculates the center of gravity point of the affect area sends the point immediately to Base Station (BS). BS processes the points and creates direction vectors to estimate the fire spreading direction globally.

To the best of our knowledge, the proposed fire propagation method has not been used in any other research papers. We have submitted the paper titled "Estimation of Fire Propagation Using Wireless Sensor Nodes" to 9th Annual IEEE International Conference on Pervasive Computing and Communications 2011.

The thesis is organized as follows: Chapter 2 reviews the fire detection techniques and disaster propagation estimation mechanisms. Fire detecting technique based on Fuzzy Logic Inference System is explained in Chapter 3. An innovated fire propagation estimation protocol is presented in Chapter 4. Chapter 5 is the conclusion and the future work. 


\section{Chapter 2 - Literature Review}

The emergence of Wireless Sensor Network (WSN) is one of the latest trends towards the miniaturization and ubiquity of computing devices. These devices are getting less expensive, more flexible, distributed and pervasive. Several advanced technologies, for instance the System On Chip technology is able to integrate the complete system on a single chip as well as a set of sensors, provide the necessary hardware capabilities. Wireless networking and collaborative signal processing enable the routing and data fusion technologies on the development of the Wireless Sensor Network.

\subsection{Wireless Sensor Networks overview}

The wireless sensor networks, which are monitored and controlled by the management center, consist of a data acquisition network and a data distribution network [4]. See Figure 2-1.

Sensors are the key components in the data acquisition network, which used to collect static and dynamic variables of the environment. The node is usually an integrated component of sensing, computing, communication, actuation and power. Thousands of the nodes in the WSN cooperate to share the information with BS and peers through either directly or in multi-hop connections. Comparing to running wires and cables, 
wireless network does not need any pre-existing infrastructure. It is easy to maintain, and is easy to install [24]. Base Station collects the information from sensors and performs data processing. Management Center stores the data, analyzes database and assists Base Station to make the decision.

Users in the data distribution network can interact with the sensor network by retrieving information or performing operations through the management center, which is the interface between users and sensor networks. The sensor network ultimately connects to Internet via Ethernet or Cellular Network etc., so that the database is available to share globally [54].

\section{Wireless Sensor Networks}

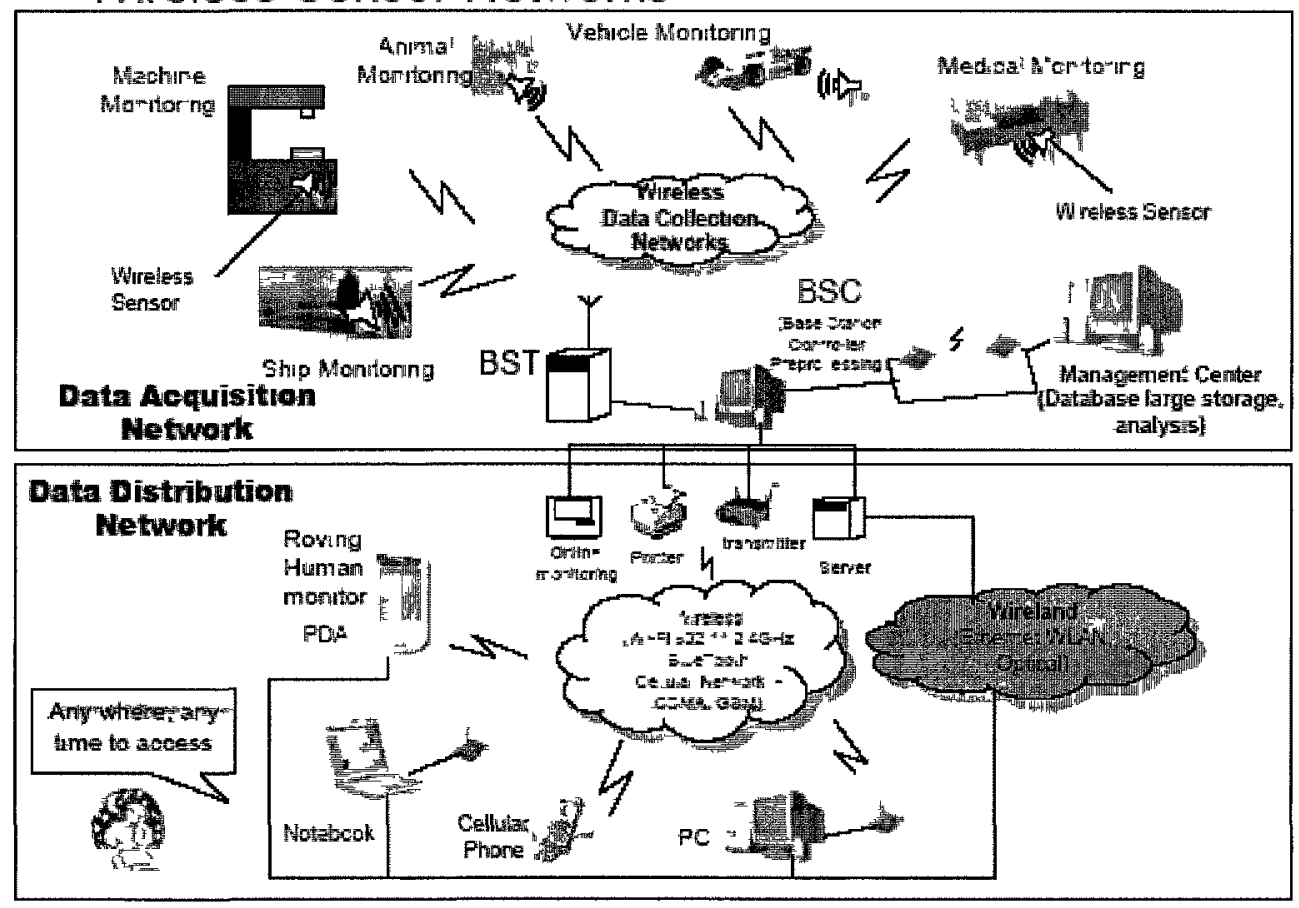

Figure 2-1: Wireless Sensor Network [4] 


\subsection{Fire Detection Techniques Overview}

Wireless sensor networks have been used widely from industrial and military to human daily life; for instance: unattended environmental and habitat monitoring [28], medical oriented health care monitoring [27], home monitoring and automation, military surveillance [26] and industrial automation and structure monitoring [29][30]. One of the most important applications of wireless sensor networks is used on disaster detection especially fire alarm detection system. There are many researches investigating the fire alarm system in various places, e.g. residential fire, forest fire and mines [13] [35] [39][40]. The studies are focused on the two areas: the best combination set of sensors

and appropriate techniques [2]. Based on the report from US National Interagency Fire Center [31], in the first ten months of 2010, there were 59,928 fires and 3,124,993 arcs area were burnt. According to National Fire Protection Association [32], in 2009, 377,000 fires occurred in residential properties and over 3000 civilians lost their lives during the fire. These number shows how crucial and significant fire detection is in our society.

The human nose is sensitive to the odors of gases and the human brain processes these scents [33] [34]. For residential fire alarm, the most common used sensors are ionization sensors and photoelectric sensors, which can detect airborne smoke [7] by mimicking the biological sense of smell. However, the false alarm rate is higher due to the nuisance sources such as cigarette or toasting bagel. An estimated $95 \%$ of all alarms reported by smoke detectors during 1980s were unnecessary. By simply increasing the àlarm threshold of conventional sensor detector has the consequence of reducing fire sensitivity. Moreover, decreasing the alarm threshold of a conventional detector turns out 
the increasing nuisance sensitivity [43]. Many experiments are focused on the study of the combinations of multiple sensors. The work of [35] conducts small-scale experiment using neural network to find the significant patterns of the fire and the environment by measuring temperature, light obscuration and $\mathrm{CO}, \mathrm{CO}_{2}, \mathrm{O}_{2}$ concentrations. These patterns are incorporated into fire detection techniques to distinguish the real fire and nuisance fire. [37] accomplishes the experiment with the improvement of fire detection and the reduction of the false alarm using the combination of smoke detector and carbon monoxide sensor. The author of [42] uses temperature, $\mathrm{CO}$ ionization and photoelectric sensors while [43] proves combining $\mathrm{CO}$ detector with temperature or ionization detector increases both fire sensitivity and nuisance immunity. The work of [41] is also applied Multi Sensor based Fire Detection (MSbFD), which uses temperature and optical smoke density sensors to collect the data. The sensor signals are evaluated and processed by fuzzy logic system. [36] conducts experiments from wide variety of fire and nuisance sources, using Principal Component Analysis (PCA), a multivariate statistical analysis, to identify the nature of source. Principal components are selected from an analysis of data ranging from one to the number of sensors. The more principal components there are, the more accurately the sources are classified, however the more complexity the algorithm becomes. By balancing the number of principal components and sufficient accuracy of detection, the author comes up the possible pair of the sensors are $\mathrm{CO}$ and $\mathrm{CO}_{2} . \mathrm{CO}_{2}$ threshold concentration or rate of rise can be used to identify flaming fires and $\mathrm{CO}$ can be used for non-flaming fires. All above researches carry out the conclusion that using multiple sensors improves fire detection and eliminates the noise detection. 
Forest fires present different constrains than indoor fires. The traditional forest fire measures are ground patrolling and watching towers using special devices Osborne Firefinder [44]. Some researches are using image assistance detection and monitor system. [45] proposes forest fire detection principle by contrasting differences between natural background and characteristics of smoke plume from image which is obtained from multiple staring black and white video camera installed in the field. [1] uses wireless sensor network mixing with IP cameras to verify the fire in rural and forest area of Spain. While the sensor detects the fire, it sends the alarms to the control center. The central server locates the closest camera and sets it towards the fire zone. Therefore the real time image can be received. This is able to help fire fighters to monitor and extinguish fire immediately. Multi-sensor nature of the technique is also applied in the forest fire detection, thus increases the possibility of detecting forest fire with high accuracy and lower false alarm. In [13], the sensor feeds the cluster head with the collected data, e.g. temperature, humidity, smoke and windy speed. The cluster head, which is treated as the hidden layer nodes in the neural network, generates the weather index based on the data, and compares with the weather index threshold to decide if the new index needs to be sent to the manager node. The manage node generates the forest fire danger rate for each cluster header based on the weather index it provides. In [39], the authors introduce FWI index and a novel k-coverage using $\mathrm{k}$ or more sensors to improve fault tolerance. Some sensors can be put in standby mode, therefore k-coverage solution prolongs the lifetime of sensor network. [48] describes a "in-suit" forest temperature measurement network developed in the forest, which includes wireless sensors network, local control node, central control node and "Firementor" software. 
Sensors collect temperature from the forest, and send to local control node via radio link. Local control node then passes data to central control node via public communication network. Sensor nodes report alarm when the temperature is over the predefined threshold $\left(55^{\circ} \mathrm{C}\right.$ in the paper). "Firementor" decision support server transfers the data from central control node and combines with Meteorological data to provide operational logistics, optimization and planning services.

Overall, most of the researches show that using multiple sensors could not only improve the fire detection accuracy but also decrease the false alarm in both residential and wild area. Some research papers use Fuzzy Logic System and Neural Network techniques at the control center or Base Station to evaluate the different factors from multiple sensors. The next 2 sections introduce the basic principle of Fuzzy Logic System and Neural Network.

\subsection{Principle of Fuzzy Logic System}

Zadeh introduced the concept of fuzzy set and fuzzy logic in 1965. Fuzzy Logic is the extension of the conventional two-valued logic to the continue interval by adding the intermediate values between absolute true and absolute false. A Fuzzy Logic System (FLS) is a non-linear mapping of an input data into an output data which consists of 4 steps: fuzzifier, fuzzy inference, rule and defuzzifier [15]. The objective of the FLS is to map the crisp input to the crisp output. Figure $2-2$ is depicted the structure of the fuzzy logic system. 
To understand fuzzy logic, the 2 sets of logic need to be mentioned. Crisp set is the membership function only takes 2 values: zero or unity. Fuzzy set defined on a universe of discourse takes on values in the interval [0,1]. Membership function that is usually denoted by the Greek letter $\mu$ ties a number to each element of the set, and provides a measure of the similarity of element in a crisp set to a fuzzy set. Fuzzifier matches the crisp input $x \in X$ into fuzzy sets, which is any collection of objects. The grade of membership of number between 0 and 1 for all its members describes a fuzzy set. Membership function can be either continuous or discrete. The membership functions are determined by users based on their experiences. The most common functions are triangular, trapezoidal that is a four-side figure with one pair of parallel sides, piecewise linear and Gaussian. The more membership functions are, the greater resolutions are achieved, however the higher computational complexity is needed [6][15][19].

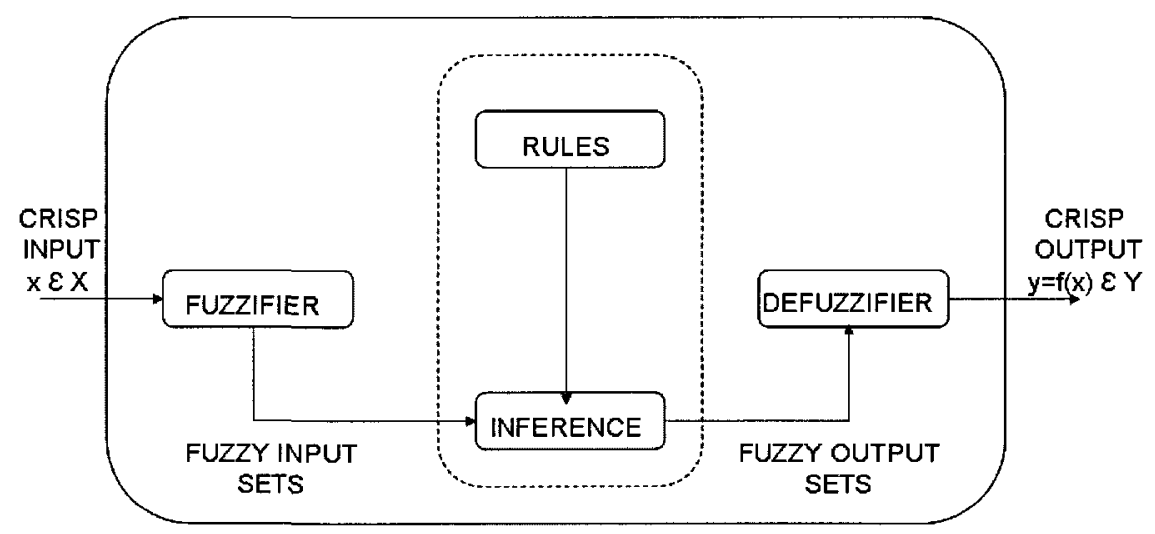

Figure 2-2: The structure of fuzzy logic system [19]

Rules are explicated as a collection of IF-THEN statements and usually built on top of expert knowledge. The linguistic variables, which are quantified by membership function, use words or sentences to describe the control system that has an antecedent block (between the IF and THEN) and a consequent block (following THEN). 
Inference engine computes fuzzy output sets by mapping the fuzzy input sets through the collection of IF-THEN rules.

IF $x_{1}$ is $F_{1}^{t}$ AND $x_{2}$ is $F_{2}^{t}$ AND $\ldots$ AND $x_{t}$ is $F_{t}^{t}$ THEN y is $G^{t}$, where $x_{t}$ is the crisp input, $F_{l}^{t}$ and $G^{t}$ are input and output fuzzy set respectively.

Defuzzifier, which is the opposite operation of Fuzzifier, converts the fuzzy output sets in to crisp numbers. There are some commonly used defuzzifiers, e.g. Maximum Defuzzifier, Centroid Defuzzifier, Height Defuzzifier etc. However no matter which defuzzification approach is chosen, the most interesting characteristic of defuzzifier in engineering application is the computational simplicity [15].

\subsection{Neural Network}

Artificial neural network (ANN) is a mathematical model based on biological neural network. The neural network acquires the network knowledge through a learning session and stores the knowledge by the weights [17]. Because of its parallel nature, the neural network has the capability of fault tolerance so that it can continue to work when an element of the neural network fails. Neural network is one of the most important data fusion techniques in the wireless sensor network. But in this thesis, the neural network is not suitable for our algorithm.

The architecture model of the neural network, which consists of neurons, is depicted in Figure 2-3. The input layer receives data from outside the neural network, hidden layer is within input layer and output layer within the neural network, and the output layer is sending the data out of the neural network. 


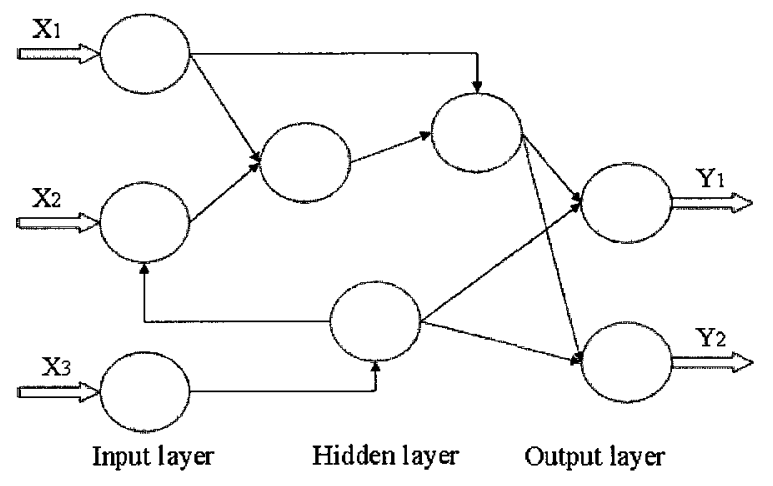

Figure 2-3: Architecture of a Neural Network

Feed forward neural network (FFNN) is the simplest ANN in which information only moves in one direction, from the input nodes, through the hidden layers to the output nodes without any feedback connections. The earliest kind of the neural network is a single-layer perceptron, which has only 1 input layer and 1 output layer. Multi-layer perceptron consists of multiple layers of neurons. Each neuron connects to the neurons in next layer.

Here is an example of a FFNN (Figure2-4) with 3 neurons in Input, 2 neurons in hidden layer and 1 neuron in output layer. The Equation 2-1 is the mathematic model of the FFNN [8]. Each neuron receives the input from external source or from neighbors. The weights are learned during the training phase by following some learning rules, gradually optimizing the performance. The sum of the product function passes the input of the previous layer as well as respective weights to the next layer [16][18].

FFNN is evaluated computationally cheap, which is very important advantage for the resources constraint sensor nodes. 


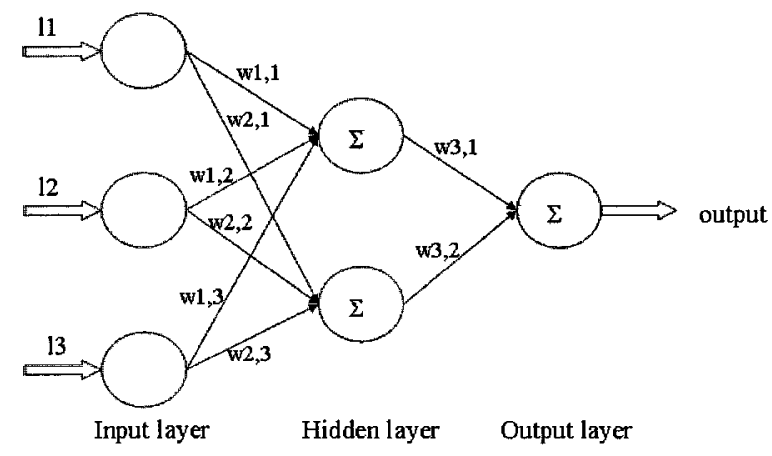

Figure 2-4: An FFNN with 3 neurons in input layer, 2 neurons in hidden layer and 1 neuron in output layer along with their weights

Output $=\left[W_{3,1} \times \sum_{J-1}^{3}\left(W_{1, j} \times I_{J}\right)\right]+\left[W_{3,2} \times \sum_{j-1}^{3}\left(W_{2, J} \times I_{J}\right)\right]$

Equation 2-1

\subsection{Applications of Fuzzy Logic System in the Wireless Sensor Networks}

Data fusion technology uses in the industrial, household, and entertainment electronics with the ability of controlling and adjusting the system and process to improve decision-making, reduce resource consumption and increase performance. Fuzzy Logic System improves the efficiency when the system has vague information and could not use the traditional mathematical model to be accurately described. It provides sensor more human-like thinking, noise-tolerant and increases the intelligence of the sensor network.

Recently quite a few researches are focused on the accuracy of the event detection and alarm application by eliminating the false alarm rate in WSN. FLS and FFNN achieve the goals with the advantages of: 1 . less development time, 2 . easily modifying 
functionality, 3. easily computing with less memory requirement, 4. tolerance of imprecise and unreliable data.

The complexity of computation of FFNN and Fuzzy Logic System are very similar and calculated by the following variables [21]:

- FFNN: $\mathrm{O}(\mathrm{m} \times \mathrm{n} \times \mathrm{p})$ where $\mathrm{m}$ is number of features, $\mathrm{n}$ is number of neurons in hidden layer and $\mathrm{p}$ is number of neurons in output layer.

- Fuzzy Logic System: $\mathrm{O}(\mathrm{m} \times \mathrm{k} \times \mathrm{r} \times \mathrm{o})$, where $\mathrm{m}$ is the number of features, $\mathrm{k}$ is number of membership functions per input, $r$ is the number of rules, $o$ is the number of outputs.

Data fusion technology at the early stage is usually applied either at cluster leader level or Base Station because they have more power and memory.

Yu et al. in [13] uses Feed Forward Neural Network at cluster leader to aggregate temperature, humidity, smoke and wind from the sensors' reading locally to generate a fire-likelihood index based on National Fire Danger Rating System for forest fire detection [47]. If the fire likelihood index does not exceed the threshold, then the cluster leader does not send message to Base Station, therefore the network overhead message is reduced significantly.

[8] assumes every node equipped four sensors based on [42] they are: temperature, ionization, photoelectric and $\mathrm{CO}$ sensors. Each sensor node feeds four sensor readings into FFNN or Naïve Bayes to determine the fire detection locally without communication with neighbors. For FFNN, when using ten hidden layers, the results get 
the highest successful rate $98.45 \%$, Naïve Bayes needs 1000 intervals to get $100 \%$ accuracy.

[50] uses wireless sensor empowered by Fuzzy Logic System in the hybrid network topology. The network consists of regular sensor nodes, sub-leader nodes, leader nodes and the master nodes. Only the sensors which detect fire transfer the data through sub-leader and leader node to the master node, rest of nodes are in the sleep mode. Sensors compare the current temperature and the temperature collected one minute ago using the Fuzzy Logic to determine the real alarm locally, consequently save the energy to transmit the unnecessary data. However, the research does not provide the data about how much false alarm is detected and how much the energy is saved.

Recently researches expand the intelligent applications to both sensor and its neighbor level to accomplish robust detection system.

[14] presents D-FLER ( a Distributed Fuzzy Logic Engine for Rule-Based Wireless Sensor Networks) which uses fuzzy logic for fusing individual and neighborhood observations. Sensor node in the experiment is either equipped with temperature and/or smoke sensors. There are four detection methods defined in order to evaluate the performance of D-FLER.

A. Threshold: Each node decides fire happened when the temperature and smoke value exceed predefined threshold. 
B. Average: Each node takes mean value between its neighbors' readings and its own samples, then compare with the predefined threshold according the algorithm mention in A.

C. Fuzzy: Each node uses local fuzzy logic engine. If the node has two sensors, four inputs are analyzed (temperature $\mathrm{T}$, temperature change $\mathrm{dT}$, smoke level S, smoke level change dS). If only one sensor is equipped, then only two parameters were analyzed. Same as A, no neighbor is involved.

D. D-FLER: based on C, input the four(or two) sensor data as well as neighborhood observations into fuzzy logic engine to make to final decision.

Both fire and non-fire cases are conducted by four detection methods, and DFLER is able to accomplish incredible results: the less than $2 \%$ error rate in the case of fire and approaching $0 \%$ in the case of nuisance tests. Because the experiments only last 60 seconds, it does not analyze the false alarm it raised.

WSN requires robust and intelligent methods to mine hidden patterns in the sensor data. Multiple features data fusion combining individual event detection and eliminating outliers and erroneous readings of individual sensor nodes increases the event detection rate and reduces false alarm rate. [21] chooses the four sensors in [8] as well. It applies two-level data fusion. The first level is at local sensor lever. The sensor node feeds the data to either FFNN or Naïve Bayes to determine the fire detection at the local level. The results are forwarded to the second level at cluster header or gateway to fuse 
second time combining the single sensor and neighbors observation. With the two level approaches, the detection accuracy rate is over $98 \%$. Moreover, it also tests the sub-set of the sensor nodes in the first level data fusion to evaluate the contribution of each feature to the fire detection system. [38] extends the testing in [21] to include wild fire detection and provides the insight on finding the correlation between various features. It reduces less contributed features and optimal set of sensors that can efficiently and reliably detect fires for both residential and wild fire detection system.

[20] is the recent study to evaluate the Fuzzy Logic System in Wireless Sensor Networks fire detection system. The proposed method involves in two types of sensors: temperature and smoke detector, with which four linguistic variables are defined: temperature $(\mathrm{T})$, temperature change $(\Delta \mathrm{T})$, smoke obscuration (S), and smoke obscuration change $(\Delta \mathrm{S})$. This method requires two temperature readings and one smoke readings to make the decision. The author also incorporates "distance" variable and "time" variable into the Fuzzy Logic rules. As we know the fire probability would be higher if three sensors from same area reported alarm than only one sensor reported the alarm. Furthermore, the time differences between sensor readings also affect the confidence of the event detection, for instance with the same amount of increase, the shorter time interval is and the higher confidence of the event detection will be. The two variables contribute the six values to the linguistic rules: distance Far (F), Distant (D) and Close ( C ), and time Long (L), Medium (M) and Short (S). With all features and variables, the rule base has 6561 rules, which are very challenging for the memory limited sensor networks. The author introduces three reduction techniques to decrease the number of rules effectively while keeps the same event detection accuracy. 
A. Separating the Rule-Base: to keep the rules that only related to the type of sensor it has.

B. Combining the rules with similar outcomes: using $\leq$ or $\geq$ to combine the fuzzy set "greater than" or " smaller than" the stands with the same outcomes

C. Incomplete Rules-Base: combining the rules with consequent that are no of interest to the default rule.

[20] imports two fire test cases from NIST [52]: burning Chair and burning Matress, demonstrates fuzzy logic approach produces better results than Navie Bayes. Also the spatial checking is proved delays the fire detection about $5 \%$ of the burning Chair case and $10 \%$ of the burning mattress case. However the paper does not provide any information related to the false alarm cases using the proposed algorithm.

So far, we have analyzed the fire detection system from data collection and processing perspective. Although the early detection is important, to oversee the fire development is the key objective to help firefighters intervene promptly. In the next section, the existing fire propagation algorithm is discussed.

\subsection{Using Wireless Sensor Networks for the Estimation of Fire Propagation Direction}

While tremendous researches focus on the early detection of the disasters, the propagation prediction attracts less attention. The spread of fire between the fire starts and when the firefighters on the scene is very important to allocate the rescue resources efficiently. 
Recent wireless sensor network has been widely used in public safety, surveillance and emergency rescuer applications. During emergency response situations, decisions have to be made quickly to coordinate and allocate the available resources. [46] proposes a simulation model using the wireless sensor network to deal with the emergency evacuation of a multi-story building. The model implements the simulation environment in tall building with few available paths and escape routes with the integration of wireless sensor network. Simulation is a graph-based model with sensors installed at the graph vertices. Hazards spread between vertices along the connected edge. Each vertex has the hazard intensity and each edge indicates with hazard weight to represent the severity of the hazard and the spreading rate. With the information sent by the sensor nodes, the sink, which is located remotely, is able to provide the real time knowledge of a developing hazard to assist the evacuation and rescuer work.

The work of [11] describes the similar framework as [46], by installing wireless sensors at the path way or the intersection of the public structures, for instance subway station. The sensor nodes collect temperature, intensity of illumination and smoke from the environment to determine the fire event. The system uses middleware, which is connected to the sink terminal, to analyze the data sent by sensors and find the safe path. LED installed at the sensor node indicates the status of the path to direct evacuation of civilian or rescue work of fire fighters.

[3] presents a distributed algorithm for indoor environment to calculate the safest escape path for evacuees through the complex building. The model consists of "hazard graph" and "navigation graph", which have sensor nodes at vortex and the edges between 
them to describe the spread of hazards and speed of evacuation of persons. If an evacuee reaches a node is earlier than hazard reaching the node, the path is considered safe.

The above models are based on fixed locations of the sensors and are mostly developed in indoor building or the public architectures. For the wild area with the big amount of sensors, the above algorithms have its limitations to monitor the status of the disasters.

Sensors capture the data and the BS, which has similar function as human brain, processes the data. Without incoming data, BS can not make the correct decision. [12] protects sensors with a equipment called "Firesensorsock" from the destruction of the fire to make sure the sensor could constantly provide the data to the BS even when the fire has passed by. The results illustrate that the temperature and humidity variations in the protected socks allow us to determine the presence of a fire and to follow the evolution of the fire. [1] is to locate the closest web camera to monitor the development of the fire once the fire alarm reported.

[49] presents a scattered wireless sensor networks composed by several isolated WSNs. The sensor nodes rate their situation with three severity levels, green means normal status, yellow means the temperature is greater than $40^{\circ} \mathrm{C}$, red means the temperature is greater than $60^{\circ} \mathrm{C}$, and the sensor fails at $80^{\circ} \mathrm{C}$. When the sensor is in either "yellow" or "red" states, it will find a safe node to send its status. Mobile patrol node, which is functions as BS, travels in the fields and collects the data from the sensor nodes. Based on the severity indicator of the nodes, it depicts a network diagram. The 
areas which contain the sensor in the "red" status are considered the affected fire areas, areas which contain the sensor in "yellow" status is likely the edges of the fire.

[5] uses the simple localization technique - Monte-Carlo Box algorithm [23], grid-based algorithm, and event-triggering mechanism for forest fire detection exclusively used for forest fire detecting system. It fixes some anchor nodes, which is fixed and equipped with GPS, while attaching sensor nodes to selected animals. The algorithm is triggered when the sensor detects a temperature rise exceeding the predefined threshold, and naturally the animals are away from the affected area. The sensor previous location is recorded as the region that is calculated based on the distances (hops multiply communication radius $\mathrm{R}$ ) to all accessible anchors. The current location is determined based on previous regions combining maximum defined velocity of the animal groups. The subjects' motion paths are achieved with $60 \%$ probability of outputting a single-grid region in the small grid structure (no greater than $6 \times 6$ ).

We propose an innovated real-time fire trajectory estimation in this thesis, which will be explained in Chapter 4 .

\subsection{Implementation of Fire Detection Algorithm using Fuzzy Logic System}

Robust and intelligent data fusion techniques mine hidden patterns in the sensor data to improve event detection rate and eliminate the erroneous readings. As the previous mentioned researches are emphasized on multi sensor scenarios assisted by the data processing techniques, some recently papers [21] and [38] examine the impact of the single features (sensors). Previous researches show combination of temperature sensor, 
ionization detector and $\mathrm{CO}$ detector increases the accuracy of the fire detection and decreases the nuisance report from the real fire based on the threshold basis fire detections.

Benefits from the new technologies enables the ability for sensor node to integrate a mini-Operation System as well as simple computing capabilities. Fuzzy Logic and FFNN have proved that less compution complexities could fit the energy and memory restricted sensor networks. Recently studies incorporate the data mining technique into the fire detection system to improve the results [8] [14] [20] [21][38]. However they all inherit the multi-sensor setup. Using the Fuzzy Logic System, less features means simpler computation. In order to simplify computation, we present the algorithm, which only involves temperature sensor with the assistance of the Fuzzy Logic engine to combine the neighbor's observation. Also we have expanded the comparison between two consecutive readings to the five continuous readings to characterize the temperature development.

The temperature sensors we have used are homogenous, the simplest and the cheapest sensor. A sensor knows its exact location parameters. The algorithm consists of two criteria: temperature criterion and neighborhood agreement.

The sensor is periodically checking environmental parameters. The traditional threshold basis algorithm is to either check if the temperature exceeds the predefined threshold or if the temperature changes between the current reading and the previous reading exceeds the predefined threshold to decide if a fire starts. The difference of our temperature criterion from them is that we are checking not only the difference between current temperature reading and previous reading, but also the temperature changes of the 
previous five readings. The purpose of the extended examination is to find the trend of the temperature development because we have noticed that the temperature from the fire cases indicates the constant increasing during the fire development, and the temperature from the nuisance cases shows the fluctuations all the time even with one or two temperature spikes. Our goal is to try and find a proper algorithm to accurately interrupt the temperature trend during the fire development. Of course, this approach could be applied to other features as well.

We present the detail design and evaluation of fire detection algorithm in Chapter 3.

\subsection{Implementation of Direction Estimation Algorithm using Wireless Sensor Networks}

Although the fire detection is important, to monitor the fire development is the key point for the evacuation and rescuer tasks. Sensors are key point of WSN. They pass the information to BS so that the control center will know what has happened in the field. It is well known that sensors could be destroyed by fire and BS can miss the updates from the destroyed sensors that may impact BS to make the decision. Research [12] tests a protection equipment "Firesensorsock" to prevent the sensor from being burnt when the fire passes by, so sensors could keep sending updates to BS. The paper [5] attaches the sensor nodes on live animals which will move away when danger happens to avoid the destruction of the sensors, thus achieves the continuous update from the sensors.

While researchers are trying to protect or move sensors away from fire, we are coming up with a novel approach to make use of the burnt sensors in the static sensor 
network. The protocol we proposed is to mark the local area of the sensor where the neighbors are missing (burnt) by mutually checking the availability between sensors and their neighbors periodically. The affected area is simply represented by the center of gravity point of the area. The center of gravity point is sent to BS for the prediction.

Sensors do not exist alone in the field. They are connected to their neighbors in the communication radius $\mathrm{r}$, which defines the maximum distance radios can reach and monitor each other's status. When a sensor notices some of its neighbors are not responding to the health check, it will be alerted and report to the location of missing area to the BS. Obviously, during the fire development BS will receive many location updates that represent the area fire exposes. The trajectory of the points helps BS to demonstrate the propagation of the fire. This robust approach aims at estimating the spreading direction of the fire after it has started. Our proposed algorithm combines the local tracking at the level of a sensor node and the global tracking performed by the base station in order to identify the spatial propagation of a fire. The details of the algorithm and performance are explained in Chapter 4. 


\section{Chapter 3 - Fire Detection Algorithms using Fuzzy Logic System}

In this chapter, Fuzzy Logic Inference System is introduced to the wireless sensor network for the fire detection algorithm. The algorithm proposed in this chapter adopts two level data fusion from [21]. The first level is conducted locally inside the sensor and the second level is carried out in the neighbors. But our algorithm is implemented on the simple temperature sensor instead of a combination of the temperature and smoke sensors. At the first level our algorithm extends the temperature checking up to five previous readings. At the $2^{\text {nd }}$ level - neighborhood checking, our algorithm uses the fuzzy logic membership function from [14] to consider the neighbors' opinion. The performance of the algorithm achieves the previous studies.

We consider a homogenous sensor network, with sensors uniformly distributed in the fields. Each node knows its exact location, also is only equipped with a temperature sensor. The sensor communicates with neighbors in broadcasting fashion within its communication range: radius $\mathrm{r}$. A Base Station (BS) collects the data generated and sent by sensor, which is located remotely. 
Our proposed fire detection algorithm uses the following two criteria to determine the fire alarm.

- Temperature criterion: checking the temperature differences between the sensor readings. This criterion defines two algorithms:

- Basic algorithm: is to compare the temperature differences between the two recent consecutive readings with the predefined threshold

- Extended algorithm: extends to the five previous temperature collections and compares four temperature differences with the predefined thresholds

- Fuzzy Logic System applied neighborhood agreement: once the sensor passes the temperature checking, it will check neighbors' consensus. The more neighbors agree with it, the higher probability of fire is.

In the next two sections, we have the detailed explanation of the fire detection algorithm.

\subsection{Temperature Criterion}

As we know, sensors can detect environmental metrics, such as temperature, humidity, smoke etc. However, the sensor itself does not have knowledge of the numbers it collects. People interpret these number into to known events. We need to find the unique characteristics to precisely describe the event and translate to the sensor network. 
Fire starts from ignition, small fire will become the big fire if there are no interruptions. Understanding the environmental parameter changes during the development of a fire helps to detect a fire at early stage and put a stop to it as soon as possible.

The sensor network has limited energy. Most of the time, the sensor is in a dormant state, checking on the environmental parameters occasionally. If it notices any unusual happenings, it will switch into active state. We assume the sensor is in the active state, the transaction trigger is not considered in this thesis.

As we known the energy used in processing the data at local sensor node is much less than communication transmission. To increase the accuracy of fire detection algorithm at the local level not only reduces the communication traffic in the network but can also saves the precious energy of WSN.

The commonly used threshold-basis algorithm is to check the temperature changes exceeding the predefined threshold $\Delta \mathrm{T}$ th. Sensor reads the ambient temperature once every tperiod time unit. A variable $\Delta \mathrm{T}$ is defined the difference between the current temperature and the one previously collected. $\Delta \mathrm{T}=\mathrm{T}-\mathrm{T}_{1}$, Where $\mathrm{T}$ is the current temperature, and $T_{1}$ is temperature collected tperiod time before current time.

Generally, a sensor is equipped with the limited memory to store the information it collects. In our case, sensor stores five temperature readings in its memory. We define the temperatures the node collected as follows:

$\mathrm{T}$ : The temperature of current reading at time $\mathrm{t}$ 
$\mathrm{T}_{1}$ : The temperature reading at time $\mathrm{t}-\mathrm{tperiod}$

$\mathrm{T} 2$ : The temperature reading at time $\mathrm{t}-2$ tperiod

T3: The temperature reading at time $\mathrm{t}-3$ tperiod

T4: The temperature reading at time $\mathrm{t}-4$ tperiod

The corresponding temperature changes are defined below:

$\Delta \mathrm{T}=\mathrm{T}-\mathrm{T}_{1}:$ The temperature changes between time $\mathrm{t}$ and the time $\mathrm{t}-\mathrm{t}$ period

$\Delta \mathrm{T}_{1}=\mathrm{T}_{2}-\mathrm{T}_{1}:$ The temperature changes between time $\mathrm{t}-\mathrm{t}_{\text {period }}$ and $\mathrm{t}-2 \mathrm{t}_{\text {period }}$

$\Delta \mathrm{T}_{2}=\mathrm{T}_{3}-\mathrm{T}_{2}:$ The temperature changes between time $\mathrm{t}-2 \mathrm{t}_{\text {perod }}$ and $\mathrm{t}-3 \mathrm{t}_{\text {period }}$

$\Delta \mathrm{T}_{3}=\mathrm{T}_{4}-\mathrm{T}_{3}:$ The temperature changes between time $\mathrm{t}-3 \mathrm{t}_{\text {period }}$ and $\mathrm{t}-4 \mathrm{tperiod}$

Therefore, the thresholds of each sampling period are defined as $\Delta \mathrm{T}_{\mathrm{th}}, \Delta \mathrm{T}_{\mathrm{th}}, \Delta \mathrm{T}$ th2, and $\Delta \mathrm{T}_{\mathrm{th}} 3$ respectively. The values of the thresholds would be defined based on expertise knowledge of fire developing characteristics. We define the two values of the state of temperature $\left(\mathrm{ST}_{\mathrm{n}}\right)$ as: High $(\mathrm{H})$ and Low $(\mathrm{L})$.

- If $\Delta \mathrm{T}_{\mathrm{n}}>\Delta \mathrm{T}_{\mathrm{thn}}$, Then $\mathrm{ST}=\mathrm{n}$

- If $\Delta \mathrm{T}_{\mathrm{n}}<\Delta \mathrm{T}_{\text {thn, }}$, Then $\mathrm{ST}_{\mathrm{n}}=\mathrm{L}$

\subsubsection{Basic Algorithm}

The basic algorithm compares the most recent temperature change $\Delta \mathrm{T}$ with the predefined threshold $\Delta \mathrm{T}_{\text {th }}$ 
IF $\Delta \mathrm{T}>\Delta \mathrm{T}$ th, THEN ST is $\mathrm{H}$, Otherwise $\mathrm{ST}$ is $\mathrm{L}$.

Also if $\mathrm{ST}$ is $\mathrm{H}$, the sensor decides the fire has happened and starts to query the neighbors. Otherwise the sensor will not go to the next level.

\subsubsection{Extended Algorithm}

This approach extends the temporal check to the previous five readings: $T, T_{1}, T_{2}$, $T_{3}, T_{4}$, and the four temperature differences between them: $\Delta T, \Delta T_{1}, \Delta T_{2}, \Delta T_{3}$

If $\Delta \mathrm{T}>\Delta \mathrm{T}$ th, Then $\mathrm{ST}=\mathrm{H}$, otherwise, $\mathrm{ST}=\mathrm{L}$.

If $\Delta \mathrm{T}_{1}>\Delta \mathrm{T}_{\mathrm{th}}$, Then $\mathrm{ST}_{1}=\mathrm{H}$, otherwise, $\mathrm{ST}_{1}=\mathrm{L}$.

If $\Delta \mathrm{T}_{2}>\Delta \mathrm{T}_{\mathrm{th} 2}$, Then $\mathrm{ST}_{2}=\mathrm{H}$, otherwise, $\mathrm{ST}_{2}=\mathrm{L}$.

If $\Delta \mathrm{T}_{3}>\Delta \mathrm{T}_{\mathrm{th}} 3$, Then $\mathrm{ST}_{3}=\mathrm{H}$, otherwise, $\mathrm{ST}_{3}=\mathrm{L}$.

In this approach, we are applying the $\mathrm{ST}_{\mathrm{n}}$ sets through the simple IF-THEN statements to perform the local evaluation. With the combination of $\mathrm{H}$ and $\mathrm{L}$ of each temperature differences, there will be $2^{4}$ rules. In this thesis, we define the two outcomes: True and False. "True" meaning the local node decides there is a fire, while "False" meaning the local node decides there is not a fire.

In this algorithm, we only define one true outcome situation: IF ST is H, AND, $\mathrm{ST}_{1}$ is $\mathrm{H}, \mathrm{AND}, \mathrm{ST}_{2}$ is $\mathrm{H}, \mathrm{AND} \mathrm{ST}_{3}$ is $\mathrm{H}$, THEN the outcome is True. The rest of the combinations infer to the "false" outcome. The rule base is defined in the table 3-1. 
Table 3-1: A fire detection rule base

\begin{tabular}{|c|c|c|c|c|}
\hline ST & $\mathrm{ST}_{1}$ & $\mathrm{ST}_{2}$ & $\mathrm{ST}_{3}$ & Outcome \\
\hline $\mathrm{H}$ & $\mathrm{H}$ & $\mathrm{H}$ & $\mathrm{H}$ & $\mathrm{T}$ \\
\hline $\mathrm{H}$ & $\mathrm{H}$ & $\mathrm{H}$ & $\mathrm{L}$ & $\mathrm{F}$ \\
\hline $\mathrm{H}$ & $\mathrm{H}$ & $\mathrm{L}$ & $\mathrm{H}$ & $\mathrm{F}$ \\
\hline \multicolumn{5}{|c|}{$\ldots \ldots$} \\
\hline $\mathrm{L}$ & $\mathrm{L}$ & $\mathrm{L}$ & $\mathrm{L}$ & $\mathrm{F}$ \\
\hline
\end{tabular}

Similar to incomplete rule described in section 2.7 [20], we define the default rule that combines all the false outcomes. Therefore, the total rule base is reduced to only two.

In the simulation program, we define thresholds are positive, which means if the temperature changes keep increasing, we conclude that fire has happened. This is also compliance with the fact that during the fire development, temperature keeps increasing.

\subsection{Neighborhood Agreement with Fuzzy Logic System}

If the decision is only based on the single sensor readings, it is prone to the false alarms and the imprecise readings. This step is to enforce the accuracy by getting the consensus from the neighborhood. Obviously, if more than one sensor detects the same alarm in the close area, the possibility of the event occurrence is definitely increased. Consequently it improves the system noise immunity and tolerance of errors.

\subsubsection{Neighborhood Agreement Algorithm}

When a sensor detects the fire alarm, instead of sending out a fire alarm to BS, it needs to confirm with its neighbors. It first sends query message to its neighbors in the communication radius $r$. If a neighbor observes the same situation, it will send a positive response to the sensor, otherwise it will send a negative response back to the sensor. 
Fuzzy Logic System assists the sensor node to make the decision based on the neighbor's responses. Figure 3-1 is depicted the procedure of the algorithm.

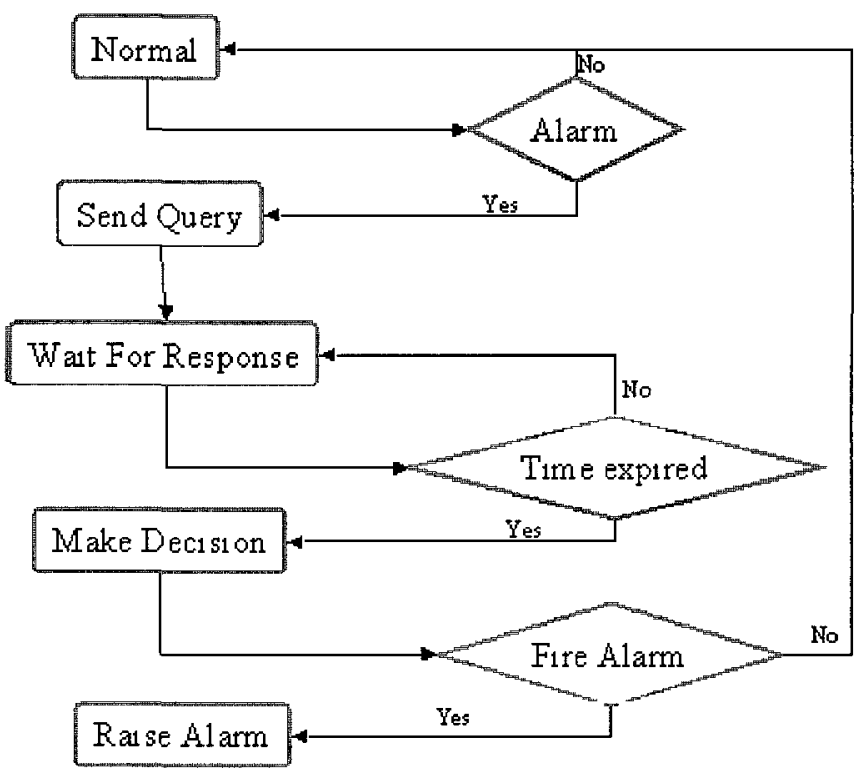

Figure 3-1: The procedure of spatial approach algorithm

1. Initially, a sensor is in the "Normal" state.

2. When a fire alarm is detected at the node level, the node sets the state to "Send Query".

3. The sensor sends query messages to neighbors, and starts to count down the waiting time Twat $_{\text {and }}$ set the state to "Wait For Response".

4. Once Twat expires, the sensor counts the positive responses and applies Fuzzy Logic Inference System and sets its state to "Making Decision". Fuzzy Logic Inference System is explained in the next section. 
5. If sensor concludes the fire has happened, it sets the state to "Raise Alarm" and sending the alarm message to BS in broadcasting fashion. Otherwise, the sensor will go back to "Normal" state.

\subsubsection{Using Fuzzy Logic Inference System to Make Decision}

Unlike a traditional crispy set, Fuzzy Logic System (FLS) provides more human like thinking. The threshold basis algorithm has only two crisp values: either over the threshold or under the threshold. However in the real life, the precise decision usually does not apply to many situations. In practice, there are no deterministic boundaries such that the following statements are true all the time: whenever the temperature is larger than 60 , a fire has started; and anytime the temperature is less than 60 , there is no fire. The use of Fuzzy Logic captures the likelihood of a fire as the collected temperature increases. Instead of setting one threshold, FLS characterizes the neighborhood observations through membership function $\mu(\mathrm{x})$, which gives fuzzy indication of the consensual neighborhood. The membership function $\mu(\mathrm{x})$ is defined in the Equation 3-1 below. FLS defines two bounds: Lower Bound (LB) and Upper Bound (UB). The values lower than LB is treated as definitely negative, the values higher than UB is defined as definitely positive, while values between LB and UB is determined by the membership function which are described as for instance, $80 \%$ positive, or $20 \%$ negative.

$\mu(x)=\left\{\begin{array}{llc}0 & \text { if } & x \leq L B \\ \frac{x-L B}{U B-L B} & \text { if } & L B \leq x \leq U B \\ 1 & \text { if } & x \geq U B\end{array}\right.$ 
The membership function is adopted from [14]. We define $\mathrm{x}$ as the percentage of the positive response. Membership function is used to characterize the neighbor's observation is defined below, and Figure 3-2.

The rules are defined as follows:

- IF less than LB percentage of neighbors consents, THEN the sensor will not report the fire alarm.

- IF more than LB and less than HB percentage of neighbors consent, THEN the sensor will calculate with the probability of confidence based on the membership function and decide if the fire has started, e.g. if $50 \%$ of neighbors see the same alarm, then there will be $40 \%$ probability of confidence that fire has happened.

- IF more than HB percentage of neighbors consents, THEN the sensor will send the fire alarm to BS immediately.

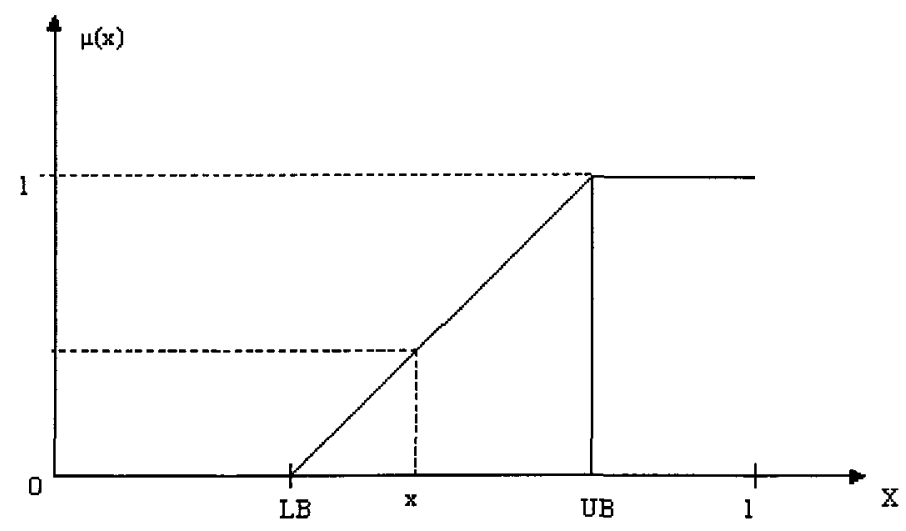

Figure 3-2: Spatial approach membership function 
Checking the neighbors' observation increases the sensor's confidence when making decision. Sensor's reading is prone to the errors and inaccuracy. If only few nodes consent, there are very likely to be errors or false alarms. If majority nodes consent, then there is a very high probability that a fire has happened.

\subsection{Definition of the Algorithms}

In the next section, we are going to present the simulation results of proposed algorithm with both fire cases and false alarm cases. A good algorithm is not only detecting fire efficiently and accurately but also immunizing false alarms. Since our first level check contains two algorithms, we divide the proposed algorithm into the two algorithms:

- Algorithm 1: is a basic algorithm with the combination of the neighbors' observation

- Algorithm 2: is an extended algorithm with the combination of the neighbors' observation

Both algorithms are tested by fire cases and false alarm cases. The time that the first sensor raises the alarm is recorded. The time takes to route the alarm to BS is not considered in the thesis. If there is no alarm time recorded, that means the algorithm does not detect a fire.

\subsection{Performance Analysis}

In spite of many simulation softwares available in the research area for instance MatLab [55] a high-level language for technical computing, Network Simulator 
(NS)[53], a discrete event simulator for simulation of TCP routing and multicast protocols over wired and wireless networks. The simulation we used in the thesis is Java based open source Mason Library [51]. MASON is a fast, portable and fairly small discrete-event multi-agent simulation library, which provides more than enough functionality for many lightweight simulation needs.

The data used by the simulation is imported from Building and Fire Research Laboratory Home Alarm Test in NIST (National Institute of Standards and Technology) [52], which is also used by papers [8] [14] [21] and [38]. Tests listed in NIST are conducted in actual homes with representative sizes and floor plans, utilized actual furnishings and household items for fire sources. Some fire alarm cases are conducted as well as some important nuisance alarm cases.

A manufactured home is procured and delivered to the NIST site for both fire and nuisance test to ensure the test environment represents the real dwellings. Thermocouples trees are arranged in the room of origin, remote bedroom, and egress path to collect the temperature data. Thermocouples are distributed evenly from floor to ceiling vertically. To simplify the simulation model, we only pick the data from the top thermocouple (20 $\mathrm{mm}$ from ceiling) of the origin location as the input data of the simulation because the temperatures at other positions are in the same pattern only with the different delays. See Figure $3-3$ is the temperature data in the living room $20 \mathrm{~mm}$ from the ceiling and Figure $3-4$ is the temperature in living room, $300 \mathrm{~mm}$ from the ceiling.

Figure 3-3 temperature reaches the highest point slightly earlier than Figure 3-4. The highest temperatures in the 2 figures are about $100^{\circ} \mathrm{C}$ difference. Figure 3-3 has the 
highest temperature over $500^{\circ} \mathrm{C}$, while Figure 3-4 has the highest only over $400^{\circ} \mathrm{C}$. But as long as the temperature is over $100^{\circ}$, it will not impact the sensor's behavior very much as the sensor will not be functional at any temperatures over $100^{\circ} \mathrm{C}$. The most important point is that both diagrams are in the same pattern which means when the temperature increases in the Figure 3-3 from time 0 s to time around $225 \mathrm{~s}$, the temperature in Figure 3-4 behaves the same way, only in the slight time delay that will not impact the simulation results as we are tracking the temperature developing trend instead of the value.

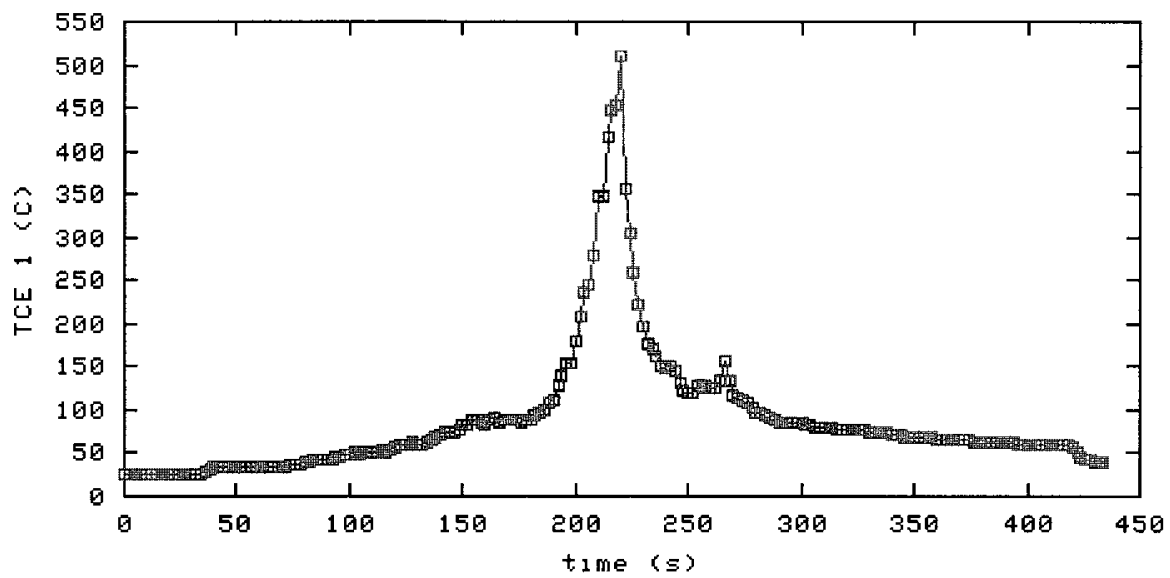

Figure 3-3: Temperature in living room, $20 \mathrm{~mm}$ from the ceiling

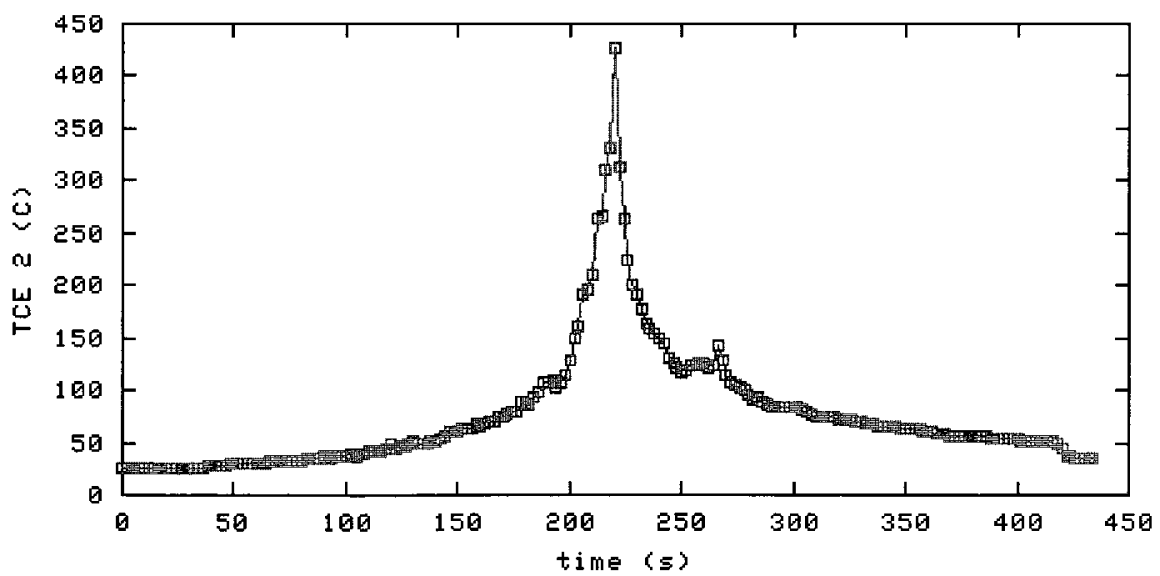

Figure 3-4: Temperature in living room, $300 \mathrm{~mm}$ from the ceiling 


\subsubsection{Simulation Environment Setup}

The simulation field defines $100 \times 100$ grid, which contains 10,000 cells. Each cell is one unit length and one unit width. A cell is identified by the bottom-left coordinates $(x, y)$, for instance, Cell( 2,3$)$ is the area $x$ between 2-3 and y between 3-4. 500 wireless sensors are randomly spread in the grid. The coordinates $(\mathrm{x}, \mathrm{y})$ of the sensor are randomly generated from $(0,100)$, therefore it is likely that there are no sensor in one cell while there are more than 1 sensors in the other cell. Assume each sensor knows its coordinates $(x, y)$, the cell in which it is located, and its unique node ID which is based on the cell it is located plus a index indicated the number of sensor in the cell. For instance, a sensor is at coordinates $(2.8,3.5)$, and it is located in cell $(2,3)$, with the node ID: 230. See Figure 3-5. The sensor is periodically collecting ambient temperature. The sensor is able to communicate with neighbor sensors around it within the circle of radius $r$, and store the neighbors' information, for instance, Node ID, and coordinates. The Base Station is located at coordinates $(100,100)$. Since the thesis is not focused on the routing of the message, we assume the sensor communicates with its peers through broadcasting message. Each sensor only broadcasts the same message once to avoid duplicating and saving the energy. All the messages defined in the simulation program are identified by the unique message ID.

\subsubsection{Temperature Propagation Model}

Temperature is based on the cell, which means there is the same temperature anywhere in the same cell. In the normal state, cell temperature is the base temperature 
with less than one degree fluctuation. The base temperature could be room temperature, or the temperature of a wild area based on the weather etc. depending on the area the wireless sensor network is developed. A fire starts randomly in one cell when simulation starts and spreads concentrically to neighbor cells evenly in all directions. Since we are using the grid to simulate the fields, to simplify the program, we define fire spreading in a square fashion rather than a circle fashion. The spreading rate uses in the simulation is one unit/second. See Figure 3-5. For instance, at time t second, temperature at cell $(2,3)$ is T1. At time $t+1$, cell $(2,3)$ updates its temperature from input data to $\mathrm{T} 2$ and temperature $\mathrm{T} 1$ spreads to the area 1 . At time $\mathrm{t}+2$, cell $(2,3)$ updates its temperature to $\mathrm{T} 3$, temperature $\mathrm{T} 2$ passes to the area 1, temperature $\mathrm{T} 1$ passes to area 2 , and so on.

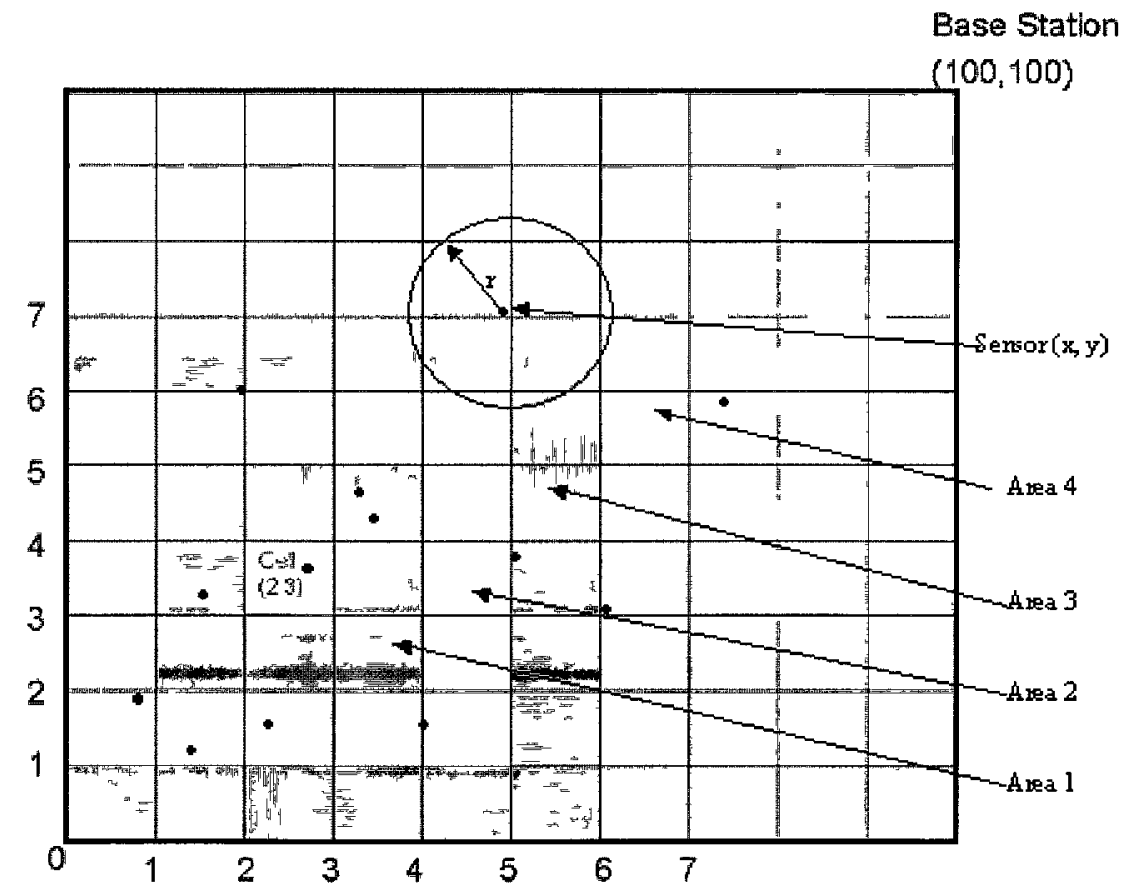

Figure 3-5: Simulation grid with the example cell $(2,3)$ and some sensor nodes with the communication radius $r$. Base Station is at $(100,100)$ 
The input data is obtained from NIST Building and Fire Research Laboratory Home Smoke Alarm Test [52]. The temperature in [52] is collected once every two seconds. See the example of the data in the Table 3-2. The fire is started at time 0 . The "time" in the table represents the period of time after the ignition. For instance, 2.000 means two seconds after the fire ignition. "tcb1", "tcd1", ..., and "tcfire" are the location of the thermocouples. In our case, only one highlighted data "tce1" is used. We also create the temperature data for the odd seconds by averaging the two adjacent even data because in our simulation the data is injected every second.

Table 3-2: Sample of the temperature data

\begin{tabular}{|c|c|c|c|c|c|c|c|}
\hline time & tcb1 & tcd1 & tcf1 & tce1 & tcc1 & tca1 & tcfire \\
\hline 0.0000 & 27.0800 & 26.8300 & 26.3300 & 25.9600 & 26.4600 & 27.2600 & 35.3900 \\
\hline 2.0000 & 27.0100 & 26.7700 & 26.4000 & 25.9600 & 26.3300 & 27.2600 & 35.4600 \\
\hline 4.0000 & 26.9500 & 26.6500 & 26.5200 & 26.0300 & 26.4600 & 27.2600 & 36.2600 \\
\hline 6.0000 & 27.0100 & 26.7100 & 26.5200 & 25.9600 & 26.5800 & 27.2000 & 39.6900 \\
\hline 8.0000 & 26.9500 & 26.7100 & 26.5200 & 26.0300 & 26.5200 & 27.2000 & 41.7100 \\
\hline
\end{tabular}

\subsubsection{Parameters in the Simulation}

The simulation starts from time 0 , and is running 3000 seconds. Fire ignition is started at time 0 as well. The simulation is tested ten times for each case, and all the alarm times displayed in the thesis are the average of ten runs unless specified. Because for each simulation run, the sensors are distributed at the beginning, and the fire is started randomly in the grid. For each run, the fire staring cell is changed and the neighbors are different. So the fire alarm time may vary a little from simulation to simulation; therefore the average time is used. For the false alarm case, some simulation runs report the alarm, some do not. The false alarm percentage is calculated based on all the simulation runs. The time that the first sensor confirms a fire has happened using the different algorithms 
is recorded respectively. Table 3-3 gives the value of parameters used in the simulation model.

Table 3-3: Parameters used in the simulation program

\begin{tabular}{|c|c|}
\hline Parameter & Value \\
\hline tperiod: sensor sampling period & $1-20$ seconds \\
\hline$\Delta \mathrm{T}_{\text {th: }}$ threshold & $1-10^{\circ} \mathrm{C}$ \\
\hline$\Delta \mathrm{T}_{\text {th1 }}, \Delta \mathrm{T}_{\text {th2 }}, \Delta \mathrm{T}_{\text {th } 3:}$ thresholds & $0^{\circ} \mathrm{C}$ \\
\hline LB: Lower Bound & 0.3 \\
\hline UB: Upper Bound & 0.8 \\
\hline Twait: time to wait for query re & 0.5 seconds \\
\hline
\end{tabular}

We have defined the first 2 parameters tperiod, which is value from 1 to 20 seconds, and $\Delta \mathrm{T}$ th, which is the value from $1-10{ }^{\circ} \mathrm{C}$ in the range, because we are trying to analysis the impact of the sampling period and the temperature thresholds in order to find efficient combinations. We have tested all the combinations, for instance, when sampling period tperıd $=1$ second, we ran the simulation with threshold $=1{ }^{\circ} \mathrm{C}, 2^{\circ} \mathrm{C}, \ldots, 10^{\circ} \mathrm{C}$ respectively. Same test for tperiod $=2$ seconds, 3 seconds, $\ldots, 20$ seconds. $\Delta T_{\text {th } 1,} \Delta \mathrm{T}_{\text {th } 2}$ and $\Delta \mathrm{T}_{\text {th } 3}$ are defined to be $0{ }^{\circ} \mathrm{C}$ as we would like to confirm the temperature increment trend in the past four sampling periods, which describes the temperature behavior during the fire development. The threshold values and the number of previous readings to be considered in the algorithm need to be optimized in order to meet the different situations in the future work. UB and LB are adopted from [14] with the value 0.8 and 0.3 meaning that if less than $30 \%$ of neighbors consensus, the sensor will not conclude the fire alarm; if more than $80 \%$ of neighbors consensus, the sensor will report the fire immediately; if the 
percentage of the consensus is between $30 \%$ and $80 \%$, the sensor will depend on Fuzzy Logic System to make the decision. The simulation is also tested with the different UB and LB in order to discover the impact of the Fuzzy Logic System. Twat is defined the time sensor is waiting for the query response from the neighbor. The radio transmits in approximately the light speed, which is $3 \times 10^{8} \mathrm{~m} / \mathrm{s}$. Therefore in the small radius range, $0.5 \mathrm{~s}$ is more than enough time to receive the response from the neighbor.

\subsubsection{Simulation Scenarios}

The test cases conducted at NIST (National Institute of Standards and Technology) Home Smoke Alarm Test [52] are selected based on a statistical analysis of available fire loss data. Two fire cases are adopted in our simulation tests: SDC10 is a living room area flaming chair and SDC36 is a bedroom flaming mattress in the manufactured home. The temperature data used in the simulation is showed in Figure 3-6 and Figure 3-8 respectively for SDC10 and SDC36.

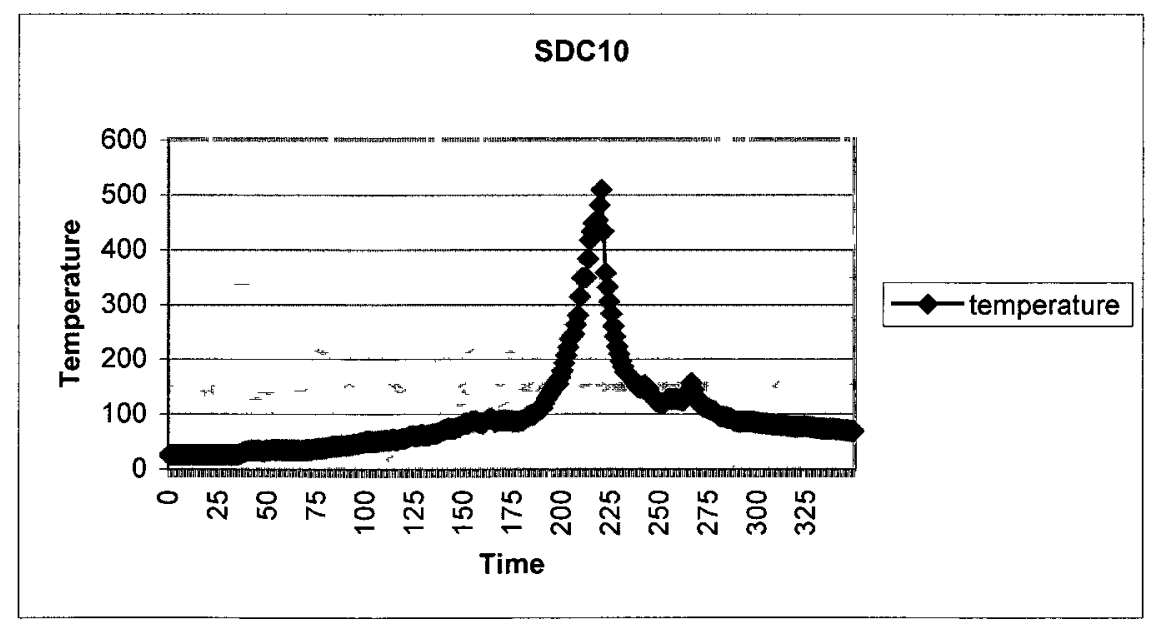

Figure 3-6: Temperature data of living room flaming chair SDC10 from 0 seconds to 345 seconds 
Figure 3-7 is the close look of the temperature from 55 seconds to 200 seconds which is clearly displayed in the temperature increasing trend.

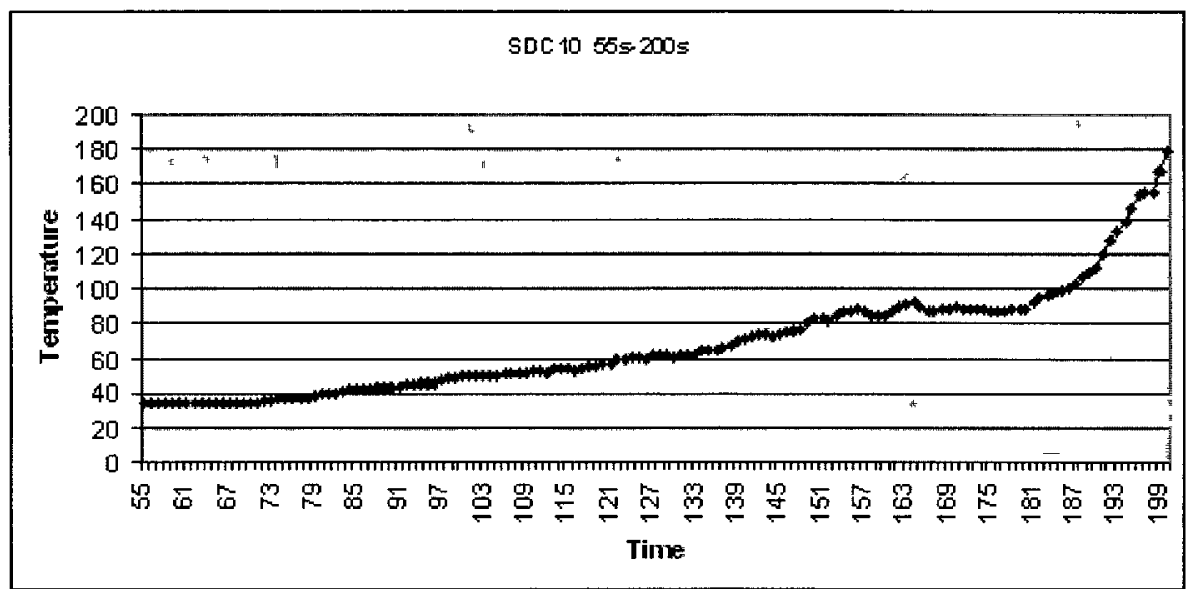

Figure 3-7: Temperature data of living room flaming chair SDC10 from 55 seconds to 200 seconds

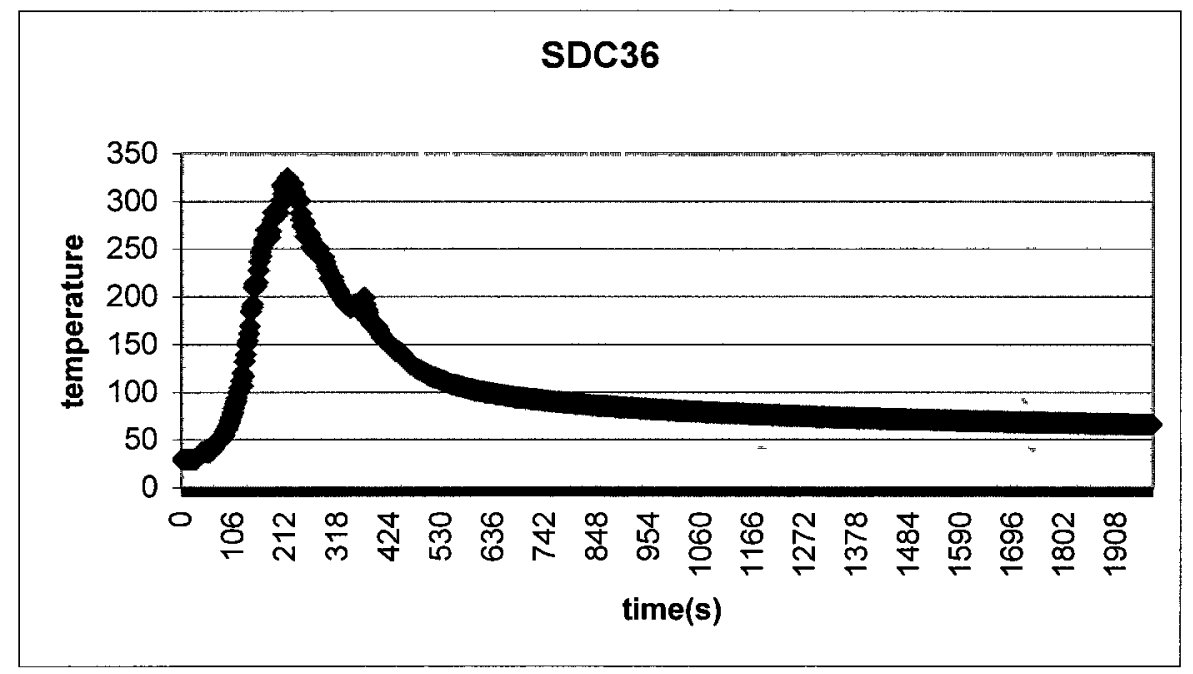

Figure 3-8: The temperature data of bedroom flaming mattress SDC36

In order to validate the algorithms, two nuisance cases are conducted. According to The Smoke Detector Operability Survey conducted by the U.S. Consumer Product Safety Commission $80 \%$ of the nuisance alarm is attributed to cooking activities. 
Therefore, we choose MNH06 a "toasting frozen bagel". The frozen bagel is toasted to medium brown color and does not char significantly. MNH33 is a "broiling frozen pizza", which has two peak temperature jumps when the oven is open. Figure 3-9 and 3-10 shows the temperature data of MNH06 and MNH33 respectively.

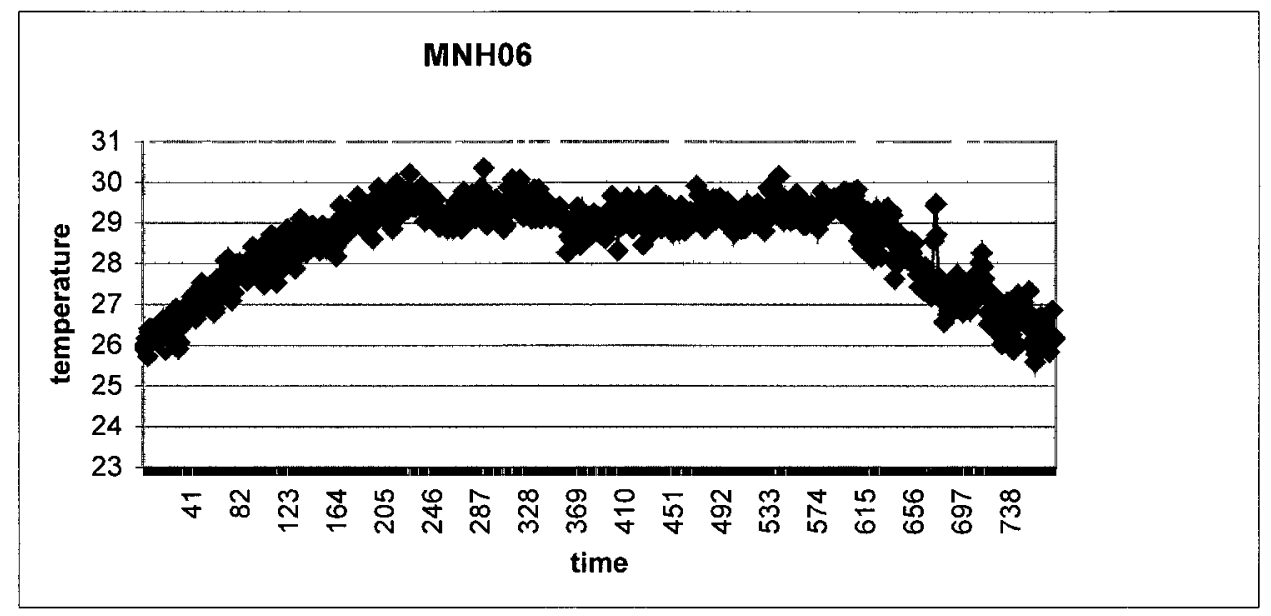

Figure 3-9: The temperature data of toasting frozen bagel MNHO

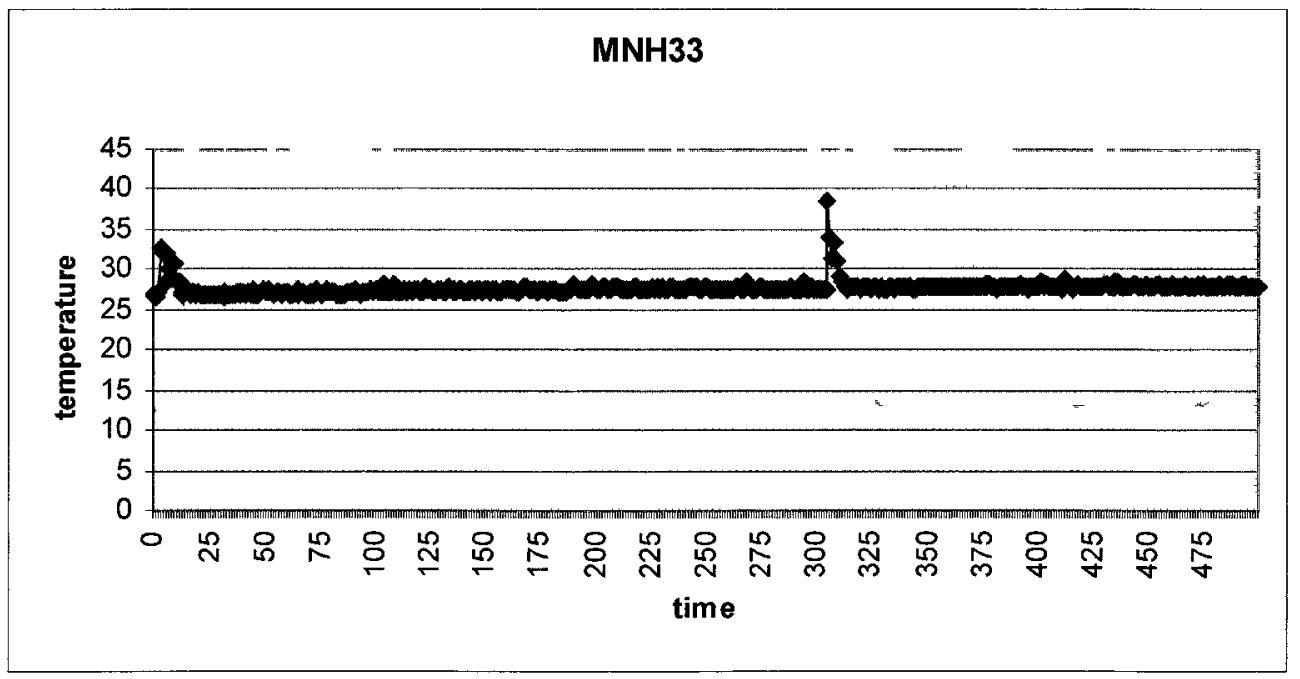

Figure 3-10: The temperature data of broiling frozen pizza MNH33 
Figure 3-11 depicts the 2 peak points in the MNH33 case, which are from 1-30 seconds and 290-320 seconds. Even though the temperature jumps at certain periods of time, it does not show the same trend as a fire case behaves.

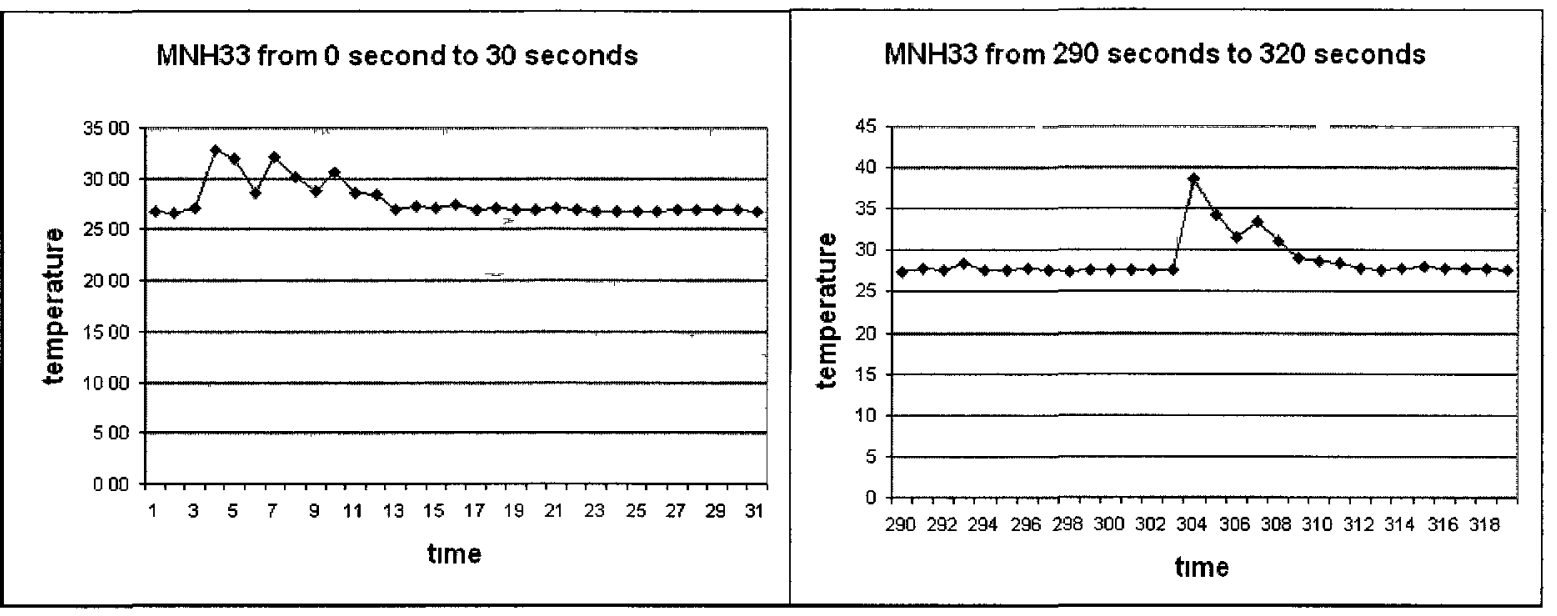

Figure 3-11: The temperature data of MNH33 in 1-30 seconds (left) and 290-320 seconds (right)

\subsubsection{Simulation Results}

The simulation testing is conducted with the parameters provided in section 3.4.3. Each test case is performed 10 times. tperiod is ranging from 1 to 20 seconds, and $\Delta \mathrm{T}_{\text {th }}$ is setting from 1 to $10^{\circ} \mathrm{C}$. We apply Algorithm 1 for the fire case and the false alarm cases.

\section{A. Algorithm 1: the basic algorithm and neighborhood agreement}

Results of testing SDC10 - "living room flaming chair" are displayed in Table 3$4,3-5$ and $3-6$. The value in the table is the time that the simulation takes to report the alarm. The unit of the value is second. The time is the average of 10 simulation runs. Some cells are empty, which means the algorithm does not detect the fire alarm. Some values show for instance 271(2), which means there are two runs out of ten detects the 
fire alarm, and the time 271 seconds is the average of the two runs. Therefore, $20 \%$ the alarm is reported, but $80 \%$ fails to detect fire.

Table 3-4 shows the results of SDC 10 using fire detection Algorithm 1 with tperiod from 1 second to 5 seconds, $\Delta \mathrm{T}$ th from $1^{\circ} \mathrm{C}$ to $10^{\circ} \mathrm{C}$. From Table 3-4, we conclude:

- The bottom left corner is almost empty, which means fire is not detected when the threshold is set too high and sampling is too frequent. Even though some cells are not empty, only some of simulations in the total ten runs detect the fire. For this fire alarm case, the totally $40.8 \%$ of runs misses to report the fire alarm.

- The higher the threshold is, the longer time takes to report the fire alarm.

Table 3-4: The results of SDC 10 with Fire Detection Algorithm 1 tperiod from $1 \mathrm{~s}$ to 5s

\begin{tabular}{c|c|c|c|c|c|}
\multirow{4}{*}{$\Delta T_{\text {th }}$} & \multicolumn{5}{|c|}{$t_{\text {period }}$} \\
\cline { 2 - 6 }$(9)$ & 1 & 2 & 3 & 4 & 5 \\
\hline 1 & 44.5 & 44.6 & 46.4 & 47.4 & 495 \\
\hline 2 & 578 & 52.2 & 49.7 & 48.2 & 49 \\
\hline 3 & 1574 & 1144 & 53 & 49 & 51 \\
\hline 4 & $185.8(5)$ & 154.2 & 75.2 & 53 & 525 \\
\hline 5 & $208.3(3)$ & 181.6 & 163.1 & 79.8 & 53.5 \\
\hline 6 & & & $19511(8)$ & 1614 & 80.5 \\
\hline 7 & & & $182(1)$ & $197(8)$ & 164 \\
\hline 8 & & & & $217(2)$ & $178.17(6)$ \\
\hline 9 & & & & & $219(2)$ \\
\hline 10 & & & & & $239(1)$ \\
\hline
\end{tabular}

Table 3-5 shows the results of SDC 10 with Fire Detection Algorithm 1 with tperiod from 6 second to 16 seconds. We summarize: 
- Almost all the runs report the fire alarm. Fire alarms miss seven times which are at the 3 cells in the bottom left corner. The failure rate is $0.64 \%$.

- While the threshold is over $8^{\circ} \mathrm{C}$, the time takes to report the fire alarm is more than doubled than the time when threshold is less than $8^{\circ} \mathrm{C}$, for instance: 59 seconds and 159 seconds. Although the alarm is detected when the threshold is set very high, it could be too late to report the fire alarm. The threshold $>8^{\circ} \mathrm{C}$ is considered not efficient.

Table 3-5: The results of SDC10 with Fire Detection Algorithm 1 tperiod from 6s to $16 \mathrm{~s}$

\begin{tabular}{|c|c|c|c|c|c|c|c|c|c|c|c|}
\hline \multirow{2}{*}{$\begin{array}{c}\Delta T_{t h} \\
(9\end{array}$} & \multicolumn{11}{|c|}{$f_{\text {penod }}$} \\
\hline & E & 7 & 8 & 9 & 10 & 11 & 12 & 13 & 14 & 15 & 16 \\
\hline 1 & 51.2 & 50.8 & 55 & 53 & 59 & 562 & 59 & 64 & 69 & 59 & 63 \\
\hline 2 & 52.4 & 55 & 55 & 539 & 59 & 617 & 60.2 & 64 & 64.8 & 62 & 63 \\
\hline 3 & 53.6 & 55 & 55 & 53.9 & 59 & 606 & 59 & 64 & 69 & 59 & 63 \\
\hline 4 & 53 & 55 & 55 & 55.7 & 59 & 60.6 & 59 & 64 & 67.6 & 64 & 63 \\
\hline 5 & 54.8 & 55.7 & 55 & 55.7 & 59 & 61.7 & 59 & 64 & 69 & 65 & 63 \\
\hline 6 & 60.8 & 56.4 & 55.8 & 59.3 & 59 & 628 & 59 & 64 & 69 & 71 & 646 \\
\hline 7 & 153.8 & 153 & 108.6 & 68.3 & 59 & 65 & 59 & 64 & 69 & 73 & 64.6 \\
\hline 8 & 164 & 157.2 & 159 & 161 & 159 & 161.8 & 1634 & 162.8 & 154.4 & 149 & 136.6 \\
\hline 9 & $189.7(9)$ & 169.8 & 164.6 & 161 & 160 & 164 & 167 & 168 & 167 & 164 & 167 \\
\hline 10 & $218(6)$ & $189.75(8)$ & 170.2 & 169.1 & 163 & 164 & 165.8 & 168 & 167 & 164 & 171.8 \\
\hline
\end{tabular}

In Table 3-6 the results show the alarm time when sampling cycle tperiod is larger than 16 seconds. The results show:

- $100 \%$ of simulation runs report the fire alarm.

- Similar to the Table 3-5, when threshold is higher than $8^{\circ} \mathrm{C}$, the alarm time is much longer than the one when threshold is lower than $8^{\circ} \mathrm{C}$. 
- The longer sampling time is using, the longer alarm time is reported.

Table 3-6: The results of SDC10 with Fire Detection Algorithm 1 tperiod from 17s to 20s

\begin{tabular}{|c|c|c|c|c|}
\hline \multirow{2}{*}{$\Delta T_{t h}$} & \multicolumn{4}{|c|}{$t_{\text {period }}$} \\
\cline { 2 - 5 }$(9$ & 17 & 18 & 19 & 20 \\
\hline 1 & 67 & 71 & 75 & 79 \\
\hline 2 & 67 & 71 & 75 & 79 \\
\hline 3 & 67 & 71 & 75 & 79 \\
\hline 4 & 67 & 71 & 75 & 79 \\
\hline 5 & 67 & 71 & 75 & 79 \\
\hline 6 & 67 & 71 & 75 & 79 \\
\hline 7 & 67 & 71 & 75 & 79 \\
\hline 8 & 123.1 & 110.6 & 88.3 & 83 \\
\hline 9 & 158.8 & 143 & 132 & 135 \\
\hline 10 & 169 & 1628 & 168.1 & 143 \\
\hline
\end{tabular}

The algorithm 1 is applied to another fire case SDC36 - "bedroom flaming mattress". The results are showed in Table 3-7, 3-8, and 3-9. By comparing Table 3-4 and Table 3-7, Table 3-5 and Table 3-8, Table 3-6 and Table 3-9, we notice the performance of the algorithm 1 is similar in these 2 fire cases. We reach the conclusion that:

- When the sensor sampling environmental data in the short period cycle (tperiod $<6$ seconds), the result is not as we expected that takes shorter time. On the contrary, it takes longer time to detect the fire, or sometimes it could not detect the fire at all. In the other words, the frequently collecting the data does not help to detect fire quickly.

- The fire alarm time is in the proportional to the threshold $\Delta \mathrm{T}$ th. The greater $\Delta \mathrm{T}_{\text {th }}$ is, the longer alarm time takes. However when the threshold is bigger 
than $8^{\circ} \mathrm{C}$, the time takes to detect the fire alarm is almost doubled than the ones that the threshold is smaller than $8^{\circ} \mathrm{C}$. It could be too late to report the fire alarm. Thus when the threshold less than $8^{\circ} \mathrm{C}$ is more efficient.

- When the sampling period is larger than 16 seconds, the alarm time is in the proportional of the sampling period tperiod.

- The successful rate of the fire detection showed in Figure 3-12. The sample period tperiod $<6$ shows lower successful rate on both fire scenarios.

Table 3-7: The results of SDC36 with Fire Detection Algorithm 1 tperiod from $1 \mathrm{~s}$ to $5 \mathrm{~s}$

\begin{tabular}{|c|c|c|c|c|c|}
\hline \multirow{3}{*}{$\Delta T_{\text {zh }}$} & \multicolumn{5}{|c|}{$t_{\text {period }}$} \\
\cline { 2 - 6 }$(9)$ & 1 & 2 & 3 & 4 & 5 \\
\hline 1 & 49.4 & 51.2 & 45.1 & 542 & 51 \\
\hline 2 & 95.4 & 82.2 & 77.3 & 57.4 & 53.5 \\
\hline 3 & 83.4 & 1056 & 107 & 93.4 & 87.5 \\
\hline 4 & 125 & 112.6 & 111.2 & 112.5 & 92.5 \\
\hline 5 & & 125.6 & 113 & 106.2 & 102 \\
\hline 6 & & 178.8 & 159.8 & 122.2 & 114.5 \\
\hline 7 & & $216.7(6)$ & & 157.8 & 127 \\
\hline 8 & & $196(2)$ & & 197.8 & 138.5 \\
\hline 9 & & $257.3(6)$ & & $197.7(9)$ & 170.5 \\
\hline
\end{tabular}

Table 3-8: The results of SDC36 with Fire Detection Algorithm 1 tperiod from 6 to 16s

\begin{tabular}{|c|c|c|c|c|c|c|c|c|c|c|c|}
\hline \multirow{2}{*}{$\Delta T_{t k}$} & \multicolumn{5}{|c|}{$t_{\text {period }}$} & \multicolumn{6}{|c|}{ (seconds) } \\
\hline & 6 & 7 & 8 & 9 & 10 & 11 & 12 & 13 & 14 & 15 & 16 \\
\hline & 51.8 & 54.3 & 55.8 & 55.7 & 59 & 58.4 & 60.2 & 62.7 & 62 & 62 & 66.2 \\
\hline 2 & 55.4 & 57.1 & 57.4 & 57.5 & 60 & 63.9 & 59 & 64 & 67.8 & 63.5 & 66.2 \\
\hline 3 & 84.8 & 62 & 61.4 & 62.9 & 60 & 68.3 & 63.8 & 64 & 69 & 69.5 & 72.6 \\
\hline 4 & 56.8 & 85.1 & 71.8 & 69.2 & 64 & 67.2 & 66.2 & 66.6 & 69 & 74 & 67.8 \\
\hline 5 & 96.8 & 91.4 & 95 & 99.8 & 95 & 80.4 & 78.2 & 73.1 & 71.8 & 78.5 & 82.2 \\
\hline$\frac{5}{6}$ & 111.2 & 105.4 & $\frac{103.8}{103}$ & 97.1 & 96 & 100.2 & 95 & 93.9 & 81.6 & 83 & 87 \\
\hline & 116.6 & 115.2 & 107 & 99.8 & 104 & 100.2 & 96.2 & 103 & 98.4 & 102.5 & 98.2 \\
\hline 8 & 1214 & 118 & 115 & 1 & 108 & 1079 & 1046 & 1056 & 1054 & 104 & 1078 \\
\hline 9 & 134.6 & 122.9 & 1206 & 120.5 & 121 & 115.6 & 109.4 & 112.1 & 106.8 & 104 & 107.8 \\
\hline
\end{tabular}


Table 3-9: The results of SDC36 with Fire Detection Algorithm 1 tperiod from 17s to 20s

\begin{tabular}{c|c|c|c|c|}
\hline \multirow{2}{*}{$\Delta T_{\text {th }}$} & \multicolumn{3}{|c|}{$t_{\text {penod }}$} & \multicolumn{3}{c|}{ (seconds) } \\
\cline { 2 - 5 }$(9$ & 17 & 18 & 19 & 20 \\
\hline 1 & 67 & 71 & 75 & 79 \\
\hline 2 & 687 & 71 & 75 & 79 \\
\hline 3 & 704 & 71 & 75 & 79 \\
\hline 4 & 738 & 71 & 75 & 79 \\
\hline 5 & 789 & 728 & 75 & 79 \\
\hline 6 & 908 & 762 & 788 & 83 \\
\hline 7 & 874 & 764 & 75 & 83 \\
\hline 8 & 1061 & 107 & 113 & 119 \\
\hline 9 & 1061 & 107 & 113 & 119 \\
\hline
\end{tabular}

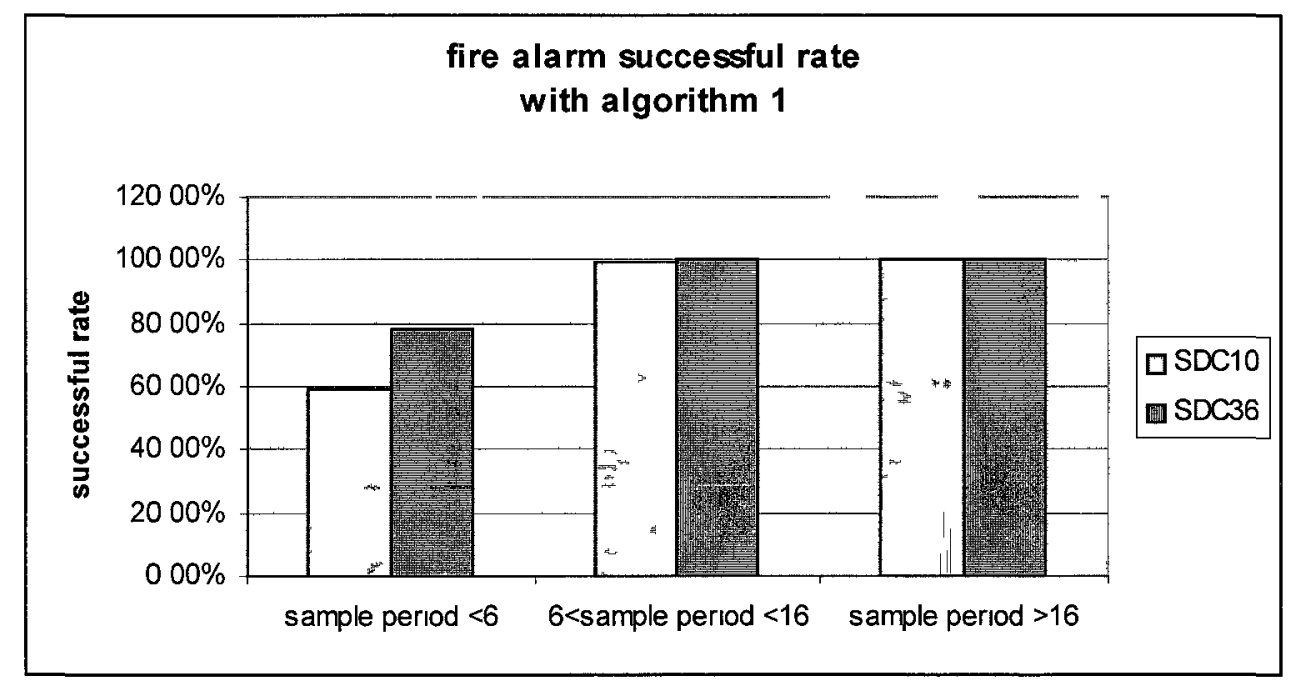

Figure 3-12: The fire detection successful rate of the 2 real fire scenarios with Algorithm 1

Based on the analysis of the fire cases, better performance occurs in the range $6<$ tperiod $<16$. In the next part nuisance testing, to simplify the comparisons, only the data in this range is displayed. Two nuisances cases have been conducted using the algorithm 1 . 
Based on the previous analysis and to clarify the comparison, we only display the results of the combinations of tperiod from 6 second to 16 seconds, $\Delta \mathrm{T}$ th from $1^{\circ} \mathrm{C}$ to $7^{\circ} \mathrm{C}$.

Table 3-10 is the results of the nuisance MNH06 - "a toasting frozen bagel". In the table, most of the cells are blank which means the algorithm does not detect the fire alarm. This is the good sign for the false alarm. The algorithm is designed to be able distinguish the real alarm from false alarm. When the threshold is $1^{\circ} \mathrm{C}$ or $2^{\circ} \mathrm{C}$, there are a total of 99 out of 200 runs detecting the false fire alarms. So the thresholds set to 1 and 2 are not immunized to the false alarm.

Table 3-10: The results of MNH06 with Algorithm 1

\begin{tabular}{|c|c|c|c|c|c|c|c|c|c|c|}
\hline \multirow{2}{*}{$\begin{array}{c}\Delta T_{t k} \\
(\mathrm{~g}\end{array}$} & \multicolumn{4}{|c|}{$\begin{array}{l}-\infty \\
t y m o\end{array}$} & \multicolumn{2}{|c|}{ (seconds) } & & \\
\hline & 6 & 7 & 8 & 9 & 10 & 11 & 12 & 13 & 14 & 15 \\
\hline 1 & 497 & 664 & 5774 & 5777 & 375 & 2355 & 101 & 1511 & $375(7)$ & $27114(7)$ \\
\hline 2 & $719(2)$ & $776(1)$ & & & & & $743(1)$ & $779(1)$ & & \\
\hline 3 & & & & & & & & & & \\
\hline 4 & & & & & & & & & & \\
\hline 5 & & & & & & & & & & \\
\hline 6 & & & & & & & & & & \\
\hline 7 & & & & & & & & & & \\
\hline 8 & & & & & & & & & & \\
\hline 9 & & & & & & & & & & \\
\hline 10 & & & & & & & & & & \\
\hline
\end{tabular}

Another false alarm case MNH33-"broiling frozen pizza" is tested with algorithm 1. The results are in the table 3-11. Unlike table 3-10, there are not many blank cells, which means the algorithm reports the false alarm most of the time. The blank cells are in the area $\Delta \mathrm{T}_{\mathrm{th}}>8^{\circ} \mathrm{C}$ area. The total false alarm rate is $55.2 \%$. 
Table 3-11: The results of MNH33 with Algorithm 1

\begin{tabular}{|c|c|c|c|c|c|c|c|c|c|c|}
\hline \multirow{2}{*}{$\Delta T_{2 k}$} & \multicolumn{10}{|c|}{$t_{\text {penod }}$} \\
\hline & 6 & 7 & 8 & 9 & 10 & 11 & 12 & 13 & 14 & 15 \\
\hline$\frac{1}{1}$ & 20 & 214 & 238 & 278 & 32 & 32 & 434 & 458 & 522 & 59 \\
\hline 2 & 212 & 207 & 27 & 35 & 42 & 529 & 71 & 116 & 90 & 1715 \\
\hline 3 & 41 & 1131 & 219 & 2681 & 332 & 3026 & 3314 & 3331 & 3392 & 332 \\
\hline 4 & 3954 & 3252 & 327 & 3311 & 340 & $34611(9)$ & 347 & $35289(9)$ & $36475(8)$ & $35338(8)$ \\
\hline 5 & 338 & 3371 & 3398 & 3527 & 345 & $34366(9)$ & $347(7)$ & $3442(9)$ & $3723(9)$ & \begin{tabular}{|l|}
$3695(8)$ \\
\end{tabular} \\
\hline 6 & $347(8)$ & $3756(5)$ & $371(6)$ & $36125(4)$ & $369(7)$ & $384(2)$ & $395(1)$ & \begin{tabular}{|c|c|}
$3604(5)$ \\
\end{tabular} & & \begin{tabular}{|c|}
$344(1)$ \\
\end{tabular} \\
\hline$\overline{7}$ & $401(2)$ & $363(1)$ & $3723(3)$ & & $319(1)$ & & & $324(1)$ & & \\
\hline 8 & & $356(1)$ & & & & $395(1)$ & & & & \\
\hline 9 & & $321(1)$ & & $366(2)$ & & & $383(1)$ & & $319(1)$ & \\
\hline 10 & $401(1)$ & & $359(1)$ & & & & $407(1)$ & $350(1)$ & & \\
\hline
\end{tabular}

Based on the experiments in the fire test cases and nuisance test cases, algorithm 1 has the following characteristics:

1. When tperiod $<6$ seconds, the algorithm misses many fire alarms. So these parameters are not considered efficient.

2. $\Delta \mathrm{T}_{\mathrm{th}}=1$ and 2 have very high false alarm reported. These two values are not immunized to the false alarm case.

3. When $\Delta \mathrm{T}_{\mathrm{th}}>7$ there are fewer false alarms reported. But based on the fire case, it takes too long time to report the alarm. It may be late to report the alarm. This area needs to be carefully used.

4. This algorithm gets very high false alarm rate $(55.2 \%)$ at one of the false alarm case MNH33, which has two temperature peaks in very short time. This algorithm works well with MNH06 which temperature changes gently instead of suddenly.

5. Overall, the parameters $2<\Delta \mathrm{T}_{\text {th }}<7$, tperiod $>5$ seconds works in both fire case, but sometimes reports some false alarm. 


\section{B. Algorithm 2: Extended Algorithm and Neighborhood Agreement}

The drawback of the algorithm 1 is that it could not handle the false alarm with the temperature jump in the short time. In this section we are using the extended algorithm to replace the basic algorithm. To simplify the comparisons, we only display the part of the results of the area that previous testing recommended $2<\Delta \mathrm{T}_{\text {th }}<7$ and $5<$ tperıd $<16$. In this section we will only use SDC10 to represent the fire case, and MNH33 to represent the false alarm case.

Table 3-12 shows the alarm time of SDC 10 with algorithm 2 with tperiod odd seconds from 6 second to 16 seconds, $\Delta \mathrm{T}$ th from $1^{\circ}$ to $7^{\circ}$. Fire alarm is reported $100 \%$ successful, which proves Algorithm 2 is applicable to the real fire alarm case.

Table 3-12: The results of SDC10 with Algorithm 2

\begin{tabular}{c|c|c|c|c|c|}
\hline \multirow{2}{*}{$\Delta T_{\text {th }}$} & \multicolumn{5}{|c|}{$t_{\text {perio }}$} \\
\cline { 2 - 6 }$(9$ & 7 & 9 & 11 & 13 & 15 \\
\hline 1 & 83.7 & 113.3 & 122.2 & 104.3 & 109 \\
\hline 2 & 106.5 & 116 & 122.2 & 106.9 & 109 \\
\hline 3 & 112.4 & 116 & 118.9 & 103 & 109 \\
\hline 4 & 113.8 & 116 & 121.1 & 1095 & 119 \\
\hline 5 & 141.1 & 1214 & 132.1 & 106.9 & 119 \\
\hline 6 & 150.9 & 1439 & 143.1 & 125.1 & 119 \\
\hline 7 & 156.5 & 158.3 & 156.3 & 144.6 & 137 \\
\hline
\end{tabular}

Table 3-13 shows the results of false alarms case MNH33 using algorithm 2 with tperiod odd seconds from 6 second to 16 seconds, $\Delta T_{\text {th }}$ from $1^{\circ}$ to $7^{\circ}$. In total 350 simulations runs, only three of them report fire alarm, while rest of them are blank which means it does not report the fire alarm. The false alarm rate is $0.857 \%$. 
Table 3-13: The results of $\mathrm{MNH33}$ with Algorithm 2

\begin{tabular}{c|c|c|c|c|c|}
\multirow{4}{*}{$\Delta T_{t h}$} & \multicolumn{5}{|c|}{$t_{\text {penod }}$} \\
\cline { 2 - 6 }$(9$ & 7 & 9 & 11 & 13 & 15 \\
\hline 1 & & & & & $689(1)$ \\
\hline 2 & & & & & $644(1)$ \\
\hline 3 & & & & & $704(1)$ \\
\hline 4 & & & & & \\
\hline 5 & & & & & \\
\hline 6 & & & & & \\
\hline 7 & & & & & \\
\hline
\end{tabular}

Based on the simulation results of one fire case SDC10 and 1 nuisance MNH33, extended temporal algorithm not only detects real fire cases $100 \%$ successfully, but also rules out the nuisance case with $0.857 \%$ failure rate. We have proved that algorithm 2 does improve the immunization to the nuisance fire cases.

\section{The impact of the extended check to the fire detection}

Fire detection algorithm 1 is only checking the most recent two sensor readings, while the algorithm 2 is checking the previous five sensor readings. The results strongly demonstrate the algorithm 2 is less impacted by the false alarm. Figure 3-13 showes the failure rate of the algorithm 1 and algorithm 2 for the nuisance case MNH33.

However, as the trade off, Algorithm 2 takes longer time than Algorithm 1 to detect a fire alarm. Table 3-14 is the comparison of the time the simulation takes to detect the fire alarm to Algorithm1 (A1) and Algorithm2 (A2) in some combinations of $\Delta T_{\text {th }}$ and tperiod. Algorithm 2 is almost doubled the fire detection time, e.g. 62.8 seconds and 143.1 seconds. 


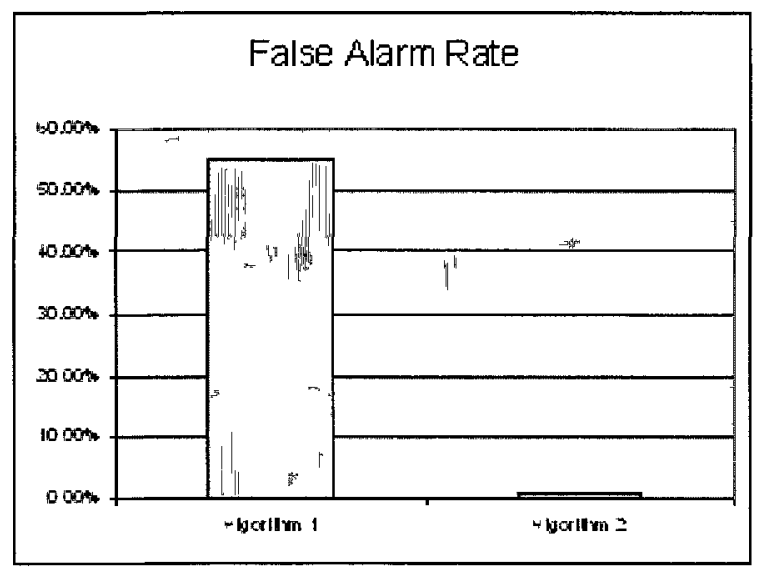

Figure 3-13: False alarm rate of MNH33 comparison between Algorithm1 and

Algorithm2

Table 3-14: Fire alarm time of SDC 10 of Algorithm1 and Algorithm2

\begin{tabular}{|c|c|c|c|c|c|c|c|c|c|c|}
\hline \multirow{2}{*}{$\frac{\Delta T_{t h}}{(9)}$} & \multicolumn{10}{|c|}{$I_{\text {perad }}$} \\
\hline & 7-A1 & $7-\mathrm{A} 2$ & 9-A1 & 9-A2 & $11-\mathrm{A} 1$ & $11-\mathrm{A} 2$ & 13-A1 & $13-A 2$ & $15-\mathrm{A} 1$ & $15-\mathrm{A} 2$ \\
\hline 1 & 508 & 837 & 53 & 1133 & 562 & 1222 & 64 & 1043 & 59 & 1085 \\
\hline 2 & 55 & 1065 & 539 & 116 & 617 & 1222 & 64 & 1069 & 62 & 1085 \\
\hline 3 & 55 & 1124 & 539 & 116 & 606 & 1189 & 64 & 103 & 59 & 1085 \\
\hline 4 & 55 & 1138 & 557 & 116 & 606 & 121.1 & 64 & 1095 & 635 & -119 \\
\hline 5 & 557 & 1411 & 557 & 1214 & 617 & 1321 & 64 & 1069 & 65 & 119 \\
\hline 6 & 564 & 1509 & 593 & 1439 & 628 & 1431 & 64 & 1251 & 71 & 119 \\
\hline 7 & 153 & $158^{45}$ & 683 & $158^{+1} 3$ & 65 & 1565 & 64 & $144^{2} 6$ & 725 & 132 \\
\hline
\end{tabular}

\section{The impact of the neighborhood observation}

To determine the contribution of neighborhood observation to the fire detection algorithm, we review the results of both Algorithm 1 and Algorithm 2 with and without neighborhood observations. For the real fire case, we investigate the successful fire alarm rate and the time the simulation takes to report the alarm. For the false alarm case, we 
inspected the false alarm rate. To simplify the comparison, we only selected tperiod $=11$ as the sample.

Table 3-15: Real fire SDC10 with and without neighborhood agreement

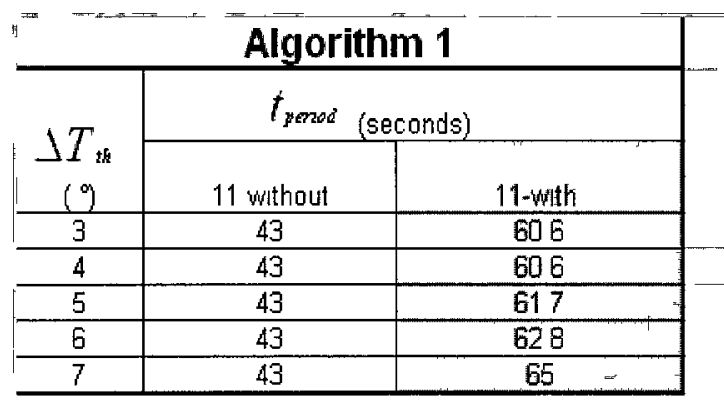

\begin{tabular}{|c|c|c|}
\hline \multicolumn{3}{|c|}{ Algorithm 2 } \\
\hline \multirow{3}{*}{$\Delta T_{t h}$} & \multicolumn{2}{|c|}{$t_{\text {penod }}$ (seconds) } \\
\cline { 2 - 3 }$(9)$ & 11 without & 11 with \\
\hline 3 & 573 & $=1189$ \\
\hline 4 & 584 & -1211 \\
\hline 5 & 532 & 1321 \\
\hline 6 & 573 & 1431 \\
\hline 7 & 573 & 1563 \\
\hline
\end{tabular}

Table 3-15 shows real fire case SDC10 with and without neighborhood agreement. Left is the Algorithm1, the right is the Algorithm 2 From Table 3-15, with neighbor's agreement takes long tıme to report the alarm in both Algorithm 1 and Algorithm 2. Algorithm 1 increases the alarm tume of $50 \%$, for instance, the time is 43 seconds without neighbors' observation, but 60.6 seconds with neighbors' observation that increases $40.9 \%$. While Algorithm 2, which is using extended temperature criterion, increases the alarm time of over $100 \%$; for instance, the time from 57.3 seconds to 1189 seconds, which increases $107.5 \%$. Both Algorithms, no matter with or without neighbors' observation, detects the fire alarm $100 \%$ successfully.

\section{Table 3-16: False alarm case MNH33 with and without neighborhood agreement}

\begin{tabular}{|c|c|c|c|c|c|}
\hline \multicolumn{3}{|c|}{ Algorithm 1} & \multicolumn{3}{|c|}{ Algorithm 2} \\
\hline \multirow{2}{*}{$\Delta T_{t}$} & \multicolumn{2}{|c|}{$t_{\text {perad }}$ (seconds) } & \multirow{2}{*}{$\begin{array}{c}\Delta T_{t h} \\
(9)\end{array}$} & \multicolumn{2}{|c|}{$t_{\text {yenod }}$ (seconds) } \\
\hline & 11 without & 11 -with & & 11 without & 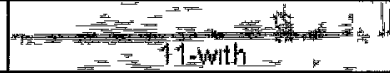 \\
\hline 3 & 21 & -3026 & 3 & & $\ldots=\ldots=-1$ \\
\hline 4 & 3103 & -3461192 & 4 & & $z_{2}=-1$ \\
\hline 5 & 3103 & $3.34366(9)$ & 5 & & = \\
\hline 6 & 3125 & $=\pi=384(2) \div \div$ & 6 & & 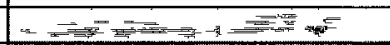 \\
\hline 7 & 3103 & 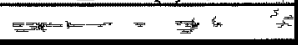 & 7 & & the \\
\hline
\end{tabular}


Table 3-16 shows false fire case MNH33 with and without neighborhood agreement. The left is the Algorithm1, the right is the Algorithm 2. Algorithm 1, with or without neighborhood checking, has very high false alarm rate. But with neighborhood checking does help to decrease the false alarm rate with about $40 \%$, but it still has $60 \%$ false alarm rate. Algorithm 2 is immunized to the false alarm case no matter with or without neighborhood checking. Also can be seen in the Figure 3-14.

Figure 3-14 shows the false alarm rate of the Algorithm 1 and 2 with or without neighbor checking. Note, for Algorithm 2, both with and without checking have $0 \%$ false alarm rate. From these comparisons, we reach the conclusion that checking neighborhood agreement does help to rule out the false alarm case, however it trade off with the longer fire detection time.

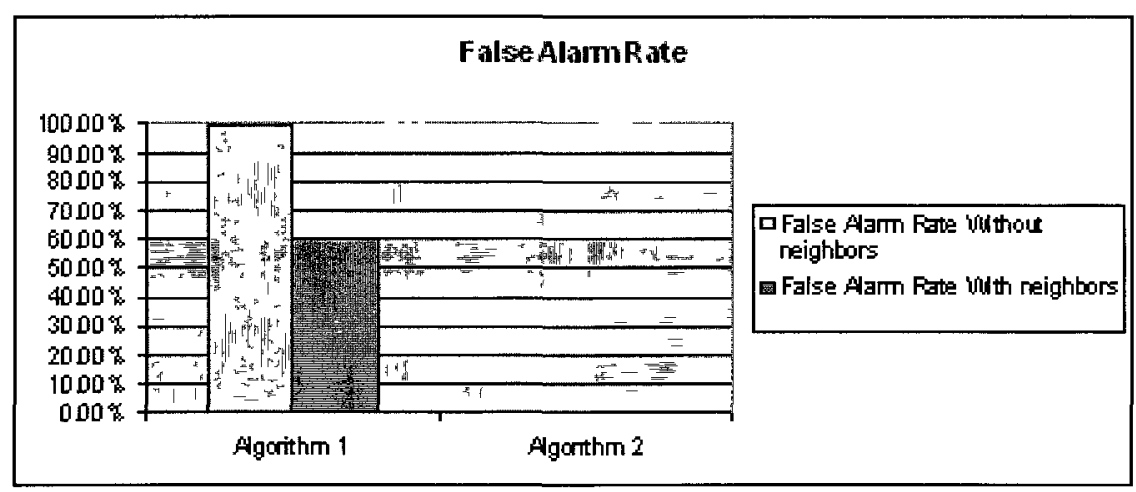

Figure 3-14: The false alarm rate of the Algorithm 1 and 2 with or without Neighborhood Checking 


\section{E. The impact of the Lower Bound and High Bound in the Fuzzy Logic System}

In the Fuzzy Logic System, membership function plays very important role. It directly impacts the sensor to make the decision. The membership function we used is defined in Equation 3-1.

$\mu(x)=\left\{\begin{array}{llc}0 & \text { if } & x \leq L B \\ \frac{x-L B}{U B-L B} & \text { if } & L B \leq x \leq U B \\ 1 & \text { if } & x \geq U B\end{array}\right.$

where $\mathrm{x}$ is the percentage of neighbors agree with the sensor. It interprets to the linguistic rules are as follows:

- If $x<L B$, which means less than $L B \%$ of neighbors agree with the sensor, the sensor will not report the alarm.

- If $\mathrm{x}>\mathrm{UB}$, which means more than $\mathrm{UB} \%$ of neighbors agree with the sensor, the sensor will raise the alarm.

- If $\mathrm{LB}<\mathrm{x}<\mathrm{UB}$, membership function $\mu(\mathrm{x})$ is depended on $\mathrm{LB}$ and $\mathrm{HB}$, which means sensor decision is depended on LB and UB.

By examining this membership function we find the relations of the parameters:

- When $\mathrm{x}$ and LB are fixed, $\mu(\mathrm{x})$ is in inverse proportional with UB. See Figure 315 Right. When $\mu(x)$ and LB are fixed, $x$ is in the proportional with UB. See Figure 3-15 Left. 
- When $x$ and UB are fixed, $\mu(x)$ is in inverse proportional with LB. See Figure 316 Right. When $\mu(\mathrm{x})$ and UB are fixed, $\mathrm{x}$ is in the proportional with LB. See Figure 3-16 Left.
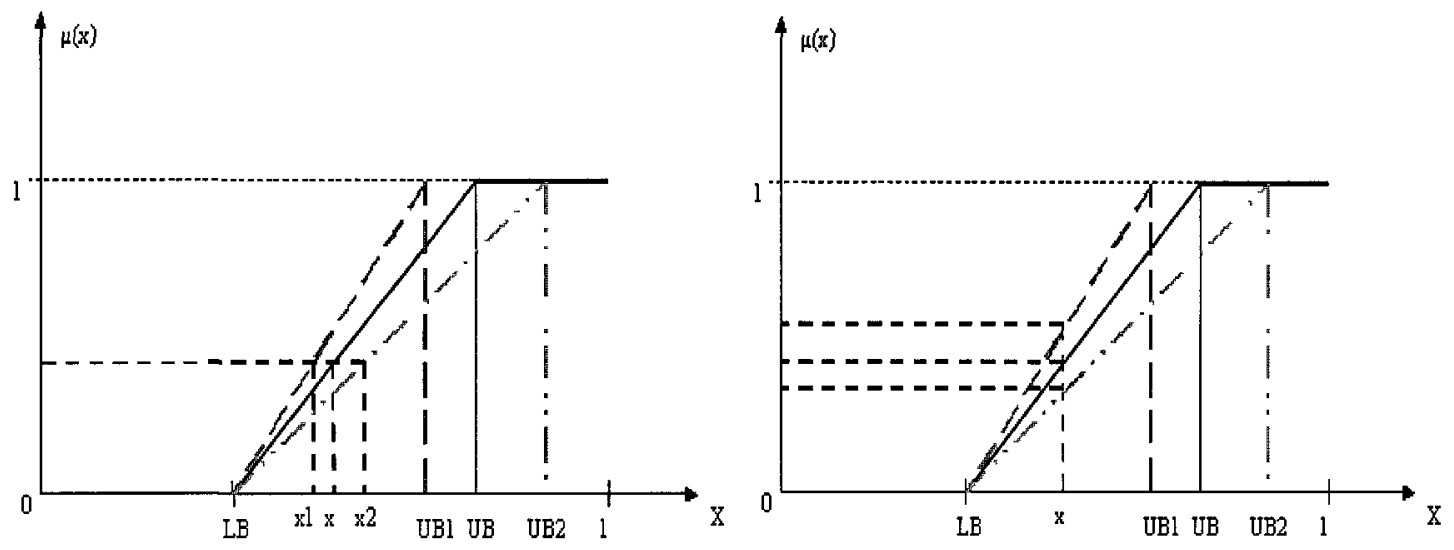

Figure 3-15: Left: the relation between $x$ and UB, Right: the relation between $\mu(x)$ and UB

To simplify the analysis, we assume both UB and LB change in the same direction. For instance, either both $\mathrm{UB}$ and $\mathrm{LB}$ are increasing or both $\mathrm{UB}$ and $\mathrm{LB}$ are decreasing. The above four statements are combined to the two statements:

- When $\mathrm{x}$ is fixed, $\mu(\mathrm{x})$ is in inverse proportional with $\mathrm{UB}$ and LB.

- When $\mu(\mathrm{x})$ is fixed, $\mathrm{x}$ is in the proportional with UB and LB.

To make the results comparable, we only think about fixing $\mu(\mathrm{x})$, which could be interpreted as achieving the same results. When UB and LB decrease, $x$ decreases, which means the sensor needs fewer neighbors' consent to achieve the same results. It could cause the more false alarms or less time to report fire alarm. On the other hand, when UB and LB increase, $x$ increases as well, which means the sensor needs more neighbors' 
consent to achieve the same results. It could cause the longer time to report the fire alarm or eliminate the false alarm.
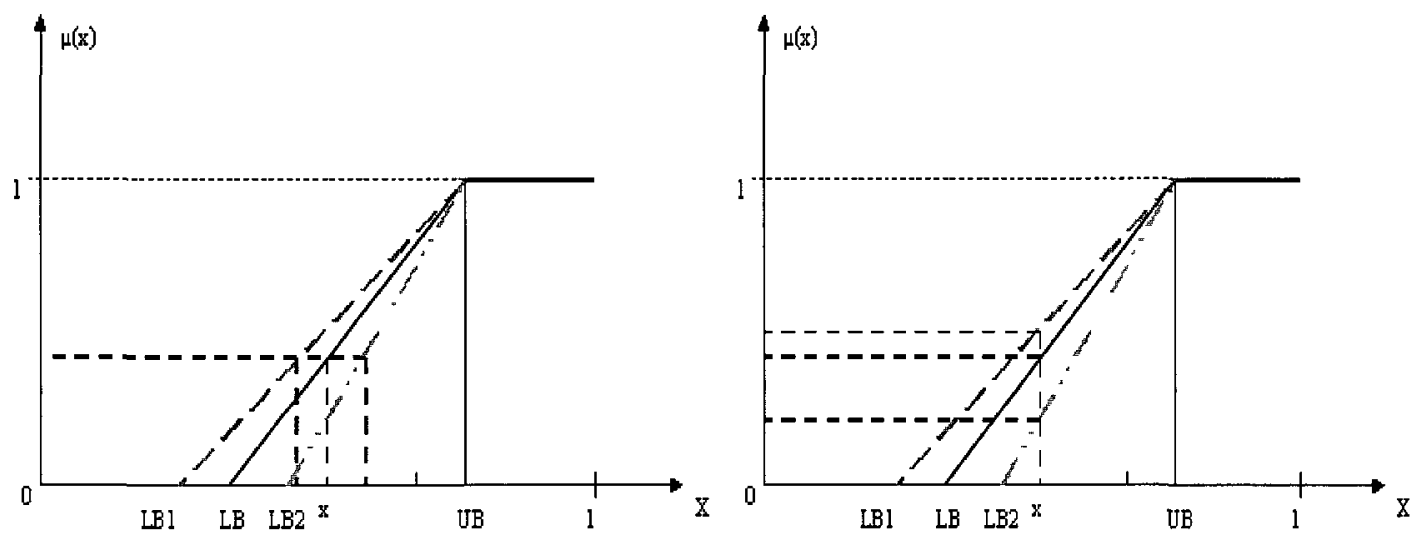

Figure 3-16: Left: the relation between $x$ and LB, Right the relation between $\mu(x)$ and $L B$

Table $3-17$ is the real fire case SDC 10 with the different LB and UB. As we expected, by decreasing both LB and UB, a sensor node needs few neighbor's consent to report the alarm, therefore in Table 3-17 less time is needed to report the alarm. Increasing both LB and UB, Table 3-17 shows the time took to report the fire alarm has also increased a lot. When $\Delta T_{t h}=7$, the alarm time is more than doubled.

Table 3-17: The alarm time of real fire scenario SDC10 with different $L B$ and UB

\begin{tabular}{|c|c|c|c|}
\hline \multicolumn{4}{|c|}{ Algorithm 1} \\
\hline \multirow{2}{*}{$\Delta T_{t s}$} & $t_{\text {periad }}$ & (seconds) & \\
\hline & $\begin{array}{c}11 \\
\mathrm{LB}=01, \mathrm{UB}=0.2\end{array}$ & $\begin{array}{c}11 \\
\mathrm{LB}=0.3 \mathrm{UB}=0.8\end{array}$ & $\begin{array}{c}11 \\
\mathrm{LB}=0.85 \mathrm{UB}=0.9\end{array}$ \\
\hline 3 & 54 & 60.6 & 66.1 \\
\hline 4 & 54 & 60.6 & 76 \\
\hline 5 & 562 & 61.7 & 74.9 \\
\hline $\bar{E}$ & 606 & 62.8 & 93.6 \\
\hline 7 & 639 & 65 & 146.4 \\
\hline
\end{tabular}


Table 3-18 is the false alarm scenario MNH33 with the different LB and UB. In Table 3-18, decreasing both LB and UB causes the more false alarms to be reported. There are much less false alarms are reported in the Table 3-18 for the false alarm scenario when increasing both LB and UB.

Table 3-18: The alarm time of false alarm scenario MNH33 with different $L B$ and UB

\begin{tabular}{|c|c|c|c|}
\hline \multirow{4}{|c|}{ Algorithm 1 } \\
\hline \multirow{3}{*}{$\Delta T_{* A}$} & $t_{\text {perid }}$ & \multicolumn{3}{|c|}{ (seconds) } \\
\cline { 2 - 4 }$(9$ & 11 & 11 & 11 \\
\hline 3 & $\mathrm{LB}=01 . \mathrm{UB}=0.2$ & $\mathrm{LB}=0.3 \mathrm{UB}=0.8$ & $\mathrm{LB}=0.85 \mathrm{UB}=0.9$ \\
\hline 4 & 32 & 302.6 & $448.6(\mathrm{~B})$ \\
\hline 5 & 33.1 & $346.11(9)$ & \\
\hline 6 & 52 & $343.66(9)$ & \\
\hline 7 & 34.3 & $384(2)$ & \\
\hline
\end{tabular}

Although the LB and UB values are less realistic, we just want to prove the impact does exist and as we expected. The parameters need to be selected carefully.

\subsection{Summary}

In this chapter, we have introduced extended temperature checking and a combination of the neighborhood agreement to improve the fire detection algorithm using the simple temperature sensor. Two fire detection algorithms are explicated and validated by the simulation program. The conclusions are:

- Sampling the environment data too often does not mean reporting the fire alarm quicker. The sampling period less than 6 seconds are considered less efficient. 
- Extended temperature checking takes longer time to report the fire alarm, however it trades off the higher noise immunity than basic temperature checking.

- Adding neighborhood checking does increase the alarm time, but the system is also reducing the number of false alarms.

- Fuzzy logic system plays the important rule in the neighborhood observation checking. The optimized parameters help systems to decrease the false alarm, and report fire alarm efficient and accurately. 


\section{Chapter 4 - Fire Propagation Estimation}

After a fire has been detected, human intervention is required immediately. To estimate the fire spreading direction will help firefighters to arrive the scene as soon as possible. As the fire in the wild area is developed randomly, any changes of wind, slope, obstacle, or type of fuels will cause the fire to spread in different directions. Most studies are focused on the early fire detection, while a few of them are related to estimation of fire development. In this chapter we propose a robust approach, which aims at estimating the spreading trajectory of the fire. Our proposed algorithm combines the local tracking at the level of a sensor node with the global tracking performed by the base station in order to identify the spatial propagation of fire.

In this chapter we will focus on the analysis of the data collected by the sensor nodes after the fire is detected. On the contrary to [5] which exploits the mobility of the devices carried by animals to track the position of the sensors and to estimate the progression of the fire, our proposed solution uses static sensor nodes by tracking the destroyed sensors to infer the trajectory of the fire. 


\subsection{Real-time Tracking Protocol}

Sensors do not exist alone in the field. They periodically query their neighbors in the communication range $r$. The protocol relies on this mutual checking to identify whose local area could have been exposed to the fire.

\subsubsection{Local Periodic Tracking}

Each sensor node is equipped with GPS and knows its location. A node A (x, y) defines its local area as a circle with radius of $r$ and centered at the node A. Any node within the distance between $\mathrm{A}$ and itself less than $\mathrm{r}$ communicates with node $\mathrm{A}$ and is collected as a neighbor of node A. Node A divides its local area into 4 sections: Local section 1 is the North-East of the local area; Local section 2 is the North-West of the local area, Local section 3 is the South-West of the local area and Local section 4 is the South-East of the local area. Neighbors are grouped into their corresponding sections. See Figure 4-1.

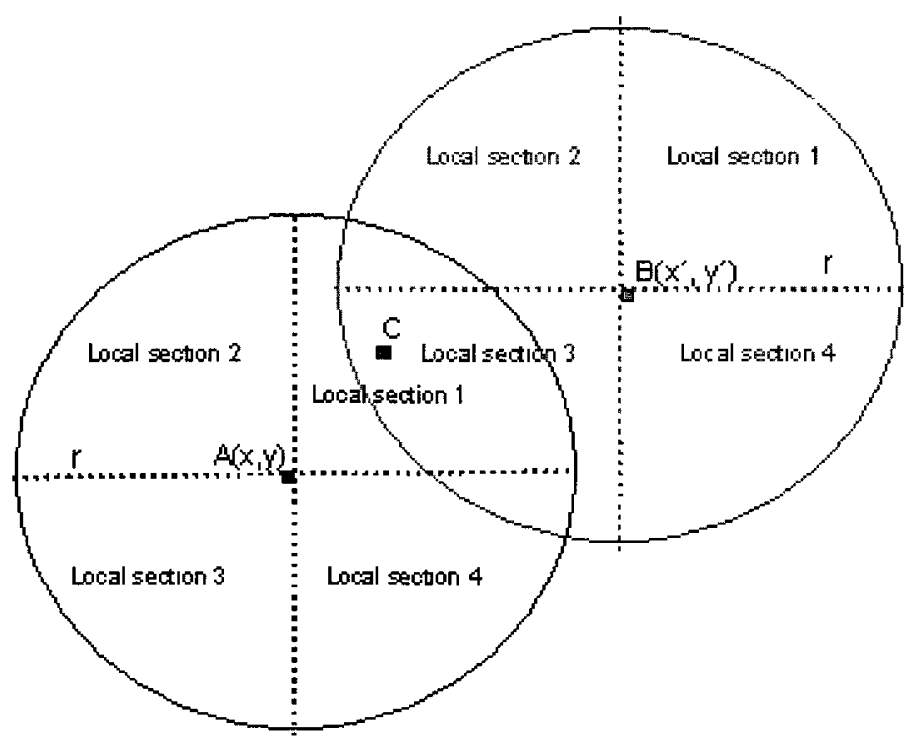

Figure 4-1: Local Section of the node A and node B 
Node $\mathrm{A}$ has the global coordinates $(\mathrm{x}, \mathrm{y})$, with node $\mathrm{C}$ as a neighbor located in the local section 1 , while node $\mathrm{C}$ is also located in the local section 3 of the node $\mathrm{B}$. On the other hand, node $\mathrm{A}$ and node $\mathrm{B}$ are the neighbors of node $\mathrm{C}$ and are located in the local section 3 and local section 1 of node $\mathrm{C}$ respectively. At the time of deployment, each node broadcasts the coordinates of its location and collects the coordinates of its neighbor within its range of communication. Neighbors are identified with the local sections with respect to the node.

Each node pings its neighbors periodically to check their status. This periodic tracking has the main task of detecting if any neighbors have been destroyed by fire. The ping message is a very simple message, which is only contains the destination nodeID. Let $\mathrm{T}$ be the ping period. Every $\mathrm{T}$ units of time, a node sends a ping message to all its neighbors and expects to receive replies from them within $\operatorname{Tr}$ units of time. The neighbor, if it is still alive, will quickly reply with the ping response message to indicate its status. We define Tmax the maximum value that $\mathrm{T}$ can take. The periodic tracking algorithm is based on rounds of ping and ping response messages. The basic idea of the checking is to find out if the node is still functional. As our assumption, when temperature is increased over $90^{\circ} \mathrm{C}$, sensor will be destroyed and not be able to respond to any messages. When a node does not receive ping response message from its neighbors, it will suspect that something probably has happened in that area. We assume sensors have enough battery during the simulation. The following paragraphs elaborate the procedure that sensor uses to determine if the local area has exposed to the fire. We define four states and each node can be in one of the following four states: "Normal", "Suspecting", "Concluding" and "Notifying". Initially, all nodes are in "Normal" state. Let's use node A as an example. 
If state="Normal":

-node A sends a round of ping message to neighbors:

If (node A receives all responses)

-set state="Normal"

-set $\mathrm{T}=\min (\mathrm{Tmax}, \mathrm{Tx} 1.1)$

If (node A misses some responses from neighbors)

-set state $=$ "Suspect"

- set $\mathrm{T}=\mathrm{T} \times 0.8$

If state="Suspect"

-node A sends a round of ping message to neighbors:

If (node A receives all responses)

-set state="Normal"

-set $\mathrm{T}=\min (\mathrm{Tmax}, \mathrm{Tx} 1.1)$

If (node A misses more responses from neighbors than last round)

-set state="Notifying"

If (node A misses the same responses from neighbors as last round)

-set state $=$ "Concluding"

-set $\mathrm{T}=\mathrm{T} \times 0.8$

If state="Concluding"

-node A sends a round of ping message to neighbors:

If (node A receives all responses)

-set state="Normal"

-set $\mathrm{T}=\min (\mathrm{Tmax}, \mathrm{Tx} 1.1)$

If (node A misses the same responses from neighbors as last round)

-set state="Notifying"

If state="Notifying"

-node A identifies the local sections that have the missing neighbors

-calculate the center of gravity of the sections

-send the center of gravity point to base station

-set state="Nomal" 
To simplify the calculation of the center of gravity, the circle area in Figure 4-1 is reformed to the square with the side of "2r" as depicted in Figure 4-2. For instance, node A has its local area in the square with the bottom-left point at (x-r, y-r) with the length of the side of $2 r$. If for instance node A concludes that its neighbors in local section 1 do not respond. The center of gravity point of section 1 is calculated at coordinates $\left(\mathrm{x}+\frac{r}{2}, \mathrm{y}+\frac{r}{2}\right)$ and sent to the BS. It is possible that the fire happens (or spreads) in more than one section. For any two adjacent sections, like section 1 and 2 , section 3 and 4 , the center of gravity point will be the center of the united rectangle. If the affected area is the combination of three sections, coordinates of node itself is used to represent the center of gravity points to simplify the process.

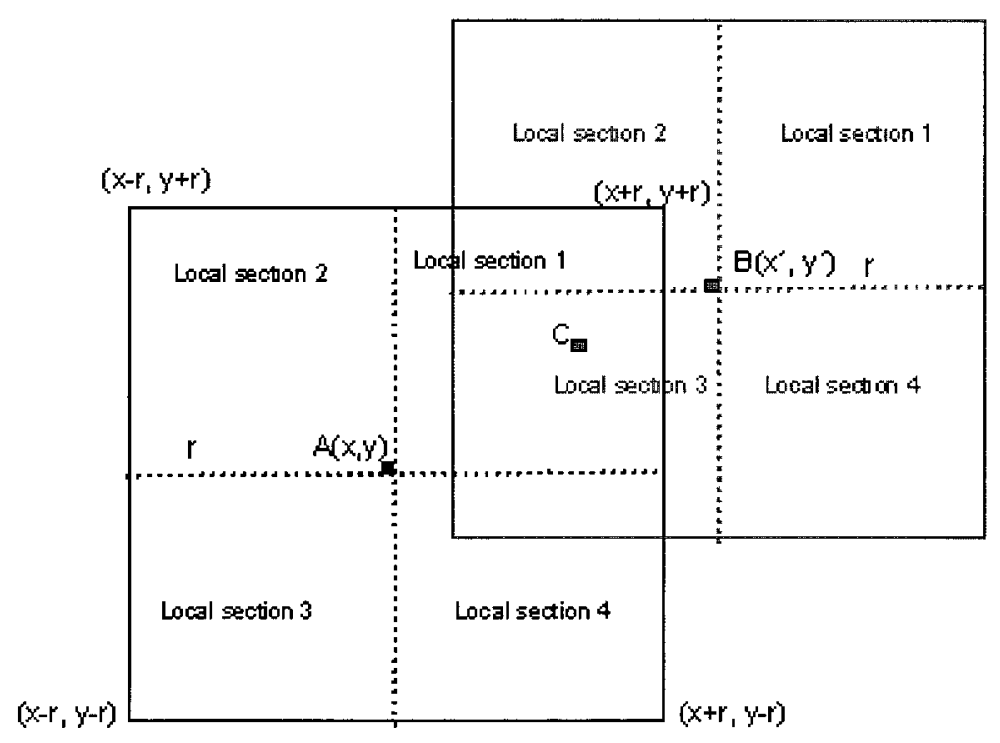

Figure 4-2: Reformed local section of the node for calculation of center of gravity point 


\subsubsection{Base Station (BS) Processing the Location Messages}

Since all the sensors are checking the status of the neighbors, the coverage is mostly overlapped. In the case where there is an actual disaster spreading in the area, it is likely that BS keeps receiving notifications from nodes which have concluded that some of their neighbors are destroyed. As the disaster propagates, BS will receive an increasing number of the center of gravity points of the area exposed to the disaster. To deal with messages efficiently, we have defined a processing time period $\mathrm{W}$ to group the incoming message based on the time and process them in the batch. Starting from the time BS receives the first fire alarm message, BS starts to collect the location messages and process them every $\mathrm{W}$ seconds time period. Each round, BS generates a location point from the data it collected and stores this point to represent the location in this round. As time goes on, BS will have a set of location points, which will be used to estimate the development of disaster.

Here is the details of how BS processing the location messages. After BS receives the first fire alarm message from the sensor, it starts to collect the center of gravity points and process them every $\mathrm{W}$ units of time. For instance, during a round $\mathrm{j}$, BS collects $\mathrm{k}$ center of gravity points of the area that exposes to the disaster: $\left(x_{1}(j), y_{1}(j)\right)$, $\left(x_{2}(j), y_{2}(j)\right), \ldots,\left(x_{k}(j), y_{k}(j)\right)$. At the end of the round, BS determines the rectangular area contains all the points it received during the round. The rectangular is presented with the bottom-left point $\left(v x_{j}, v y_{j}\right)$ and the width and length which are in the equation 4-1, 42 and $4-3$ respectively. 
$\left(v x_{j}, v y_{j}\right)=\left(\min \left(x_{1}(j), x_{2}(j), \ldots, x_{k}(j)\right), \min \left(y_{1}(j), y_{2}(j), \ldots, y_{k}(j)\right)\right) \quad$ Equation 4-1

width $=\left(\max \left(\left(x_{1}(j), x_{2}(j), \ldots, x_{k}(j)\right)-\min \left(\left(x_{1}(j), x_{2}(j), \ldots, x_{k}(j)\right)\right) \quad\right.\right.$ Equation 4-2

height $_{J}=\left(\max \left(y_{1}(j), y_{2}(j), \ldots, y_{k}(j)\right)-\min \left(y_{1}(j), y_{2}(j), \ldots, y_{k}(j)\right)\right) \quad$ Equation 4-3

To simplify the characterization of the rectangular area, BS only stores the center of gravity coordinates of the area, which is calculated by Equation 4-4.

$\left(g x_{j}, g y_{j}\right)=\left(v x_{j}+\frac{\text { width }}{2}, v y_{j}+\frac{\text { height }_{j}}{2}\right)$

Equation 4-4

Round after round, BS will obtain a series of points: $\left(g x_{0}, g y_{0}\right),\left(g x_{1}, g y_{1}\right), \ldots$, $\left(g x_{\mathrm{n}}, g y_{n}\right)$ and will use them to estimate the propagation of the disaster.

Based on the points we get, we define the propagation vector using the 2 consecutive points as follows:

$\vec{p}_{J}=\left(\left(g x_{J}-g x_{J-1}\right) ;\left(g y_{J}-g y_{J-1}\right)\right)$ for $\mathrm{j} \geq 1$

Equation 4-5

The angle associated to the vector $\vec{p}$, is calculated by Equation 4-6.

$\alpha_{J}=a \tan \left(\frac{g y_{J-}-g y_{J-1}}{g x_{J}-g x_{J-1}}\right)$

Equation 4-6

Vectors BS stored are: $\vec{p}_{1}, \vec{p}_{2}, \ldots, \vec{p}_{n}$ with angles are: $\alpha_{1}, \alpha_{2}, \ldots, \alpha_{n}$ respectively. The vectors provide the basic data to determine the fire propagation direction. 
Fire spreads freely, thus any environmental changes can cause the fire to change directions. Ideally the vectors should point to the same direction if the sensor is distributed evenly and if the fire is spreading in a certain direction. However realistically none of the vectors would have the exact same angle (direction), based on the vectors we got, we propose two algorithms: simple estimation algorithm and statistical estimation algorithm.

When the fire spreads in a non-concentric direction, in the area with evenly distributed sensor nodes, the angle difference between two consecutive vectors would be very small. This is the initial idea of the simple estimation algorithm. But for the concentrically spreading, it is hard to use a simple vector to indicate the fire direction. Therefore, we have come up with another algorithm: statistical estimation algorithm.

\section{A. Simple estimation algorithm}

In this algorithm, we define an angle threshold $\alpha_{t h}$ to measure the angle difference between 2 consecutive vectors. BS generates location points and calculates the angle of the vectors continuously after the fire starts. The angle difference of any 2 adjacent vectors is monitored closely. Once the difference of the two consecutive angles e.g. $\mid \alpha_{\text {J }}-$ $\alpha_{j-1} \mid$ is less than a predefined angle threshold $\alpha_{t h}$ in a round $\mathrm{j}$, BS will conclude the fire spreading direction is the direction of $\vec{p}_{J}$.

The simple estimation algorithm is applicable to disaster propagation constantly in the one direction. It could easily be challenged if the fire is spreading in concentric 
mode or in two opposite directions. Thus, a new algorithm - statistical estimation algorithm is introduced.

\section{B. Statistical estimation algorithm}

The algorithm is similar to the local section defined by the sensor node. BS defines four sections: section 1 is for angle $\alpha$, between 0 and $90^{\circ}$, (in other word NorthEast direction); section 2 is for angle $\alpha$, between $90^{\circ}$ and $180^{\circ}$, (North-West direction); section 3 is for angle $\alpha$, between $180^{\circ}$ and $270^{\circ}$ (South-West direction), and the section 4 is for angle $\alpha$, between $270^{\circ}$ and $360^{\circ}$ (South-East direction). BS collects the angles and maps them into one of the four sections. Fire could spread in one section, multiple sections, and parts of some sections or all sections. We propose the following rules to assist BS to make the decision. BS will calculate the percentage of collected angles in each section to determine the propagation of the disaster according to the following rules:

1. If any section contains more than $50 \%$ of the collected angles $\alpha_{J}$, BS will conclude the fire is spreading in that section. This is described as firm conclusion. For instance, if the North-East section contains more than 50\% of the collected angles, BS will conclude that the fire is spreading in the North-East area.

2. If all section (s) contain less than $50 \%$ of the angles, but one or two sections contains more than $40 \%$ of the collected angles, BS will conclude the fire is likely to spread in that direction(s). This is described as weak conclusion. 
3. If no any section contains more than $40 \%$ of the collected angles, BS concludes that the fire is spreading in all directions (concentric case).

This approach depends on the number of angles it collects. The more vectors BS collected, the better results it will get, but the longer time it will take. The results are also indirectly associated to the distribution of the sensor. Evenly distributed wireless sensor network will provide robust estimation of the disaster propagation. On the other hand, if the disaster starts in an area with few nodes, our protocol will either fail or take long time to predict.

In the next section, we present the simulation model and the performance analysis of proposed algorithms.

\subsection{Simulation Model and Assumptions}

The simulation model is similar to that of Chapter 3. The field is a $200 \times 200$ grid, which contains 40000 cells. Each cell is one unit length and one unit width. We consider a homogenous sensor network, with 2000 sensors uniformly distributed in the field. Each sensor knows its coordinates $(x, y)$, its unique nodeID, the cell it is located in and neighbors it has. A node is only equipped with temperature sensor. The sensor communicates with neighbors in broadcast mode within its communication range: radius r. Base Station (BS) located remotely at coordinates $(200,200)$, collects the data generated and sent by sensors. Please see Figure 3-5 in previous chapter as reference. We are using the fire detection algorithms 2 in the previous chapter to detect the fire. After the BS receives the first fire alarm notification, it will start to collect the location 
messages and processes them periodically. We assume the sensor will be destroyed when ambient temperature is over $90^{\circ} \mathrm{C}$.

\subsubsection{Disaster Propagation Model}

Same as previous model, the temperature is based on the cell, which means temperature is the same everywhere in a cell Unlıke the previous model, the temperature is not only spreading in concentric pattern, but also to any directions and the spreading rate is one cell every five seconds instead of one cell every second.

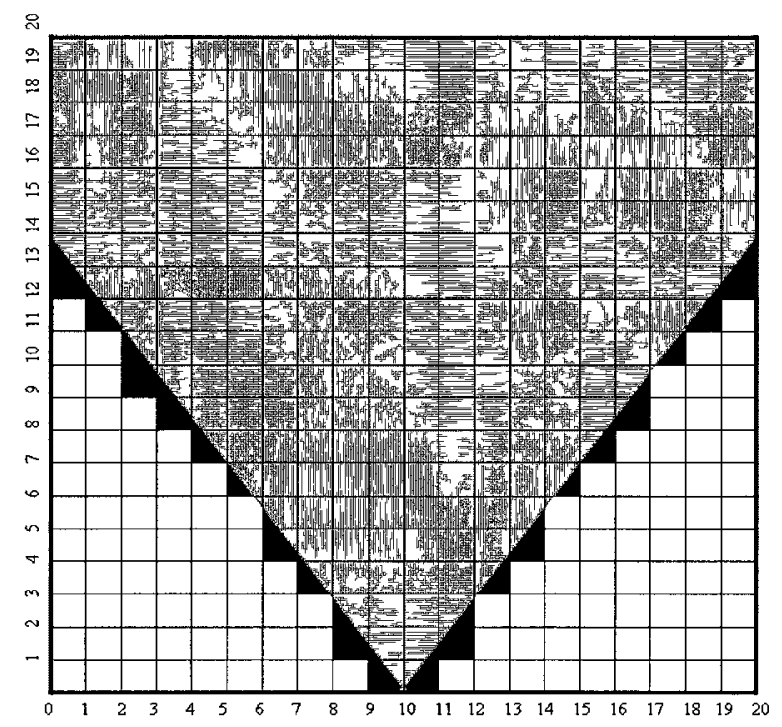

Figure 4-3 Fire spreading model example

Figure 4-3 is an example of a fire starting at $(100,0)$ and spreading to North with the $60^{\circ}$ angle. Since our temperature model is based on cells, at the border of black and dark gray areas, cells have part of area is exposed to the fire (gray area), and part of the area is not. We treat them all in the fire spreading area in our simulation model. So temperature will pass from one cell to its adjacent neighbor cells from origin cell towards 
North. The temperature in the white cells is not affected by the temperature spreading. They keep the base temperature plus $-1^{\circ}$ to $1^{\circ}$ fluctuations.

\subsubsection{Parameters in the Simulation}

The simulation is based on the Java open source Mason library [51]. The simulation uses one of the fire cases tested in previous chapter - SDC10 "living room flaming chair", which is adopted from Building and Fire Research Laboratory Home Alarm Test in NIST (National Institute of Standards and Technology) [52]. The parameters we use are in the table 4-1 below.

Table 4-1: parameters use in the simulation

\begin{tabular}{|l|l|}
\hline Parameter & Value \\
\hline W: BS processing data cycle & 30 seconds \\
\hline T: ping message cycle & 60 seconds \\
\hline Tr: waiting time for ping response & 10 seconds \\
\hline Tmax: maximum value of T & 60 seconds \\
\hline$\alpha$ th: threshold of angle difference & $15^{\circ}$ \\
\hline Maximum number of vectors & 11 or 22 \\
\hline
\end{tabular}

Sensor is sending ping message to its neighbors every 60 seconds. The sensor waits for 10 seconds to receive the ping response from neighbors. BS processing cycle $\mathrm{W}$ is defined 30 seconds which means BS calculates the location point every 30 seconds. So in statistical estimation algorithm, the more points BS needs a longer time it will take to make the decision. The angle threshold $\alpha_{t h}$ is defined $15^{\circ}$, which is only applicable in the simple estimation algorithm. When the two adjacent vectors have the angle difference 
less than $15^{\circ}, \mathrm{BS}$ will make the conclusion of the fire spreading direction. The maximum number of vectors defines two values: 11 or 22 . This parameter is designed for statistical estimation algorithm that depends on the total number of vectors it collects. The reason we pick 11 vectors is based on the performance of simple algorithm. Most of time, simple algorithm concludes the direction in around 10 vectors. In order to be comparable between 2 algorithms, we decide to use 11 vectors for statistic algorithm. In order to evaluate the impact of the number of vectors using statistical algorithm, we double the max vectors to 22 . The simulations are presented respectively in the next section.

\subsection{Performance Analysis}

The fire propagation estimation is the extension of the previous fire detection system. They are the two parts of the simulation program. First, the fire detection algorithm detects fire, and then BS starts to estimate the trajectory of the fire propagation direction. Sensors are distributed at the beginning of the simulation. Even though sensors are evenly spread in the grid, it does not guarantee in the exact same location. Therefore the simulation may have the different results. Each scenario is performed 10 times and the results are displayed in the following sections.

First of all, we use an example to explain how BS processes the message, generate the location points/vectors and determine the directions.

\subsubsection{Example of BS Determining Fire Direction}

Here is an example of how BS processes the center of gravity points, calculates the vectors and angles, and predicts the directions. The scenario we tested is that fire 
starts at $(150,150)$ and spreads towards South-West direction (shadowed area), see Figure 4-4. After BS receives the fire alarm report, it starts to collect the center of gravity points sent by sensor nodes and processes them every 30 seconds. During the first 30 seconds BS collects 9 points, see the table 4-2.

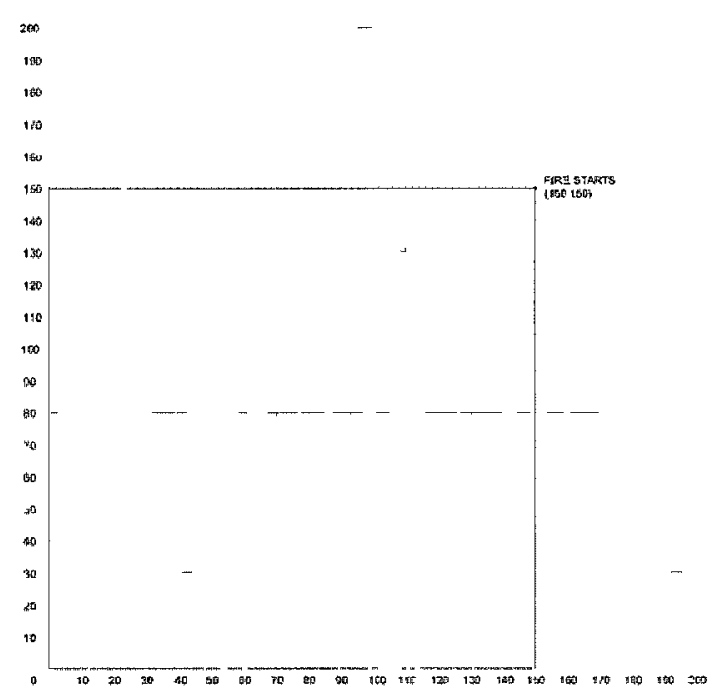

Figure 4-4: $200 \times 200$ grid with the fire starts at $(150,150)$ and spreads towards South-West direction (the shaded area)

Table 4-2: Center of gravity points $B S$ received in the first $W=30$ seconds

\begin{tabular}{|c|c|}
\hline$X(1)$ & $Y(1)$ \\
\hline 140.65 & 139.8 \\
\hline 145.44 & 148.15 \\
\hline 145.47 & 147.75 \\
\hline 146.31 & 150.74 \\
\hline 148.76 & 153.52 \\
\hline 152.97 & 152.37 \\
\hline 150.99 & 134.57 \\
\hline 13523 & 143.03 \\
\hline 147.32 & 153.72 \\
\hline
\end{tabular}


1. Using the Equation 4-1, 4-2 and 4-3, the rectangle formed by the points in table 42 is calculated the bottom-left point at: $\left(v x_{1}, v y_{1}\right)=(135.23,134.57)$, with the width $h_{1}=17.74$, and height $1=19.15$. Therefore, using the Equation $4-4$ the first point is created at $\left(g x_{0}, g y_{0}\right)=(144.1,144.15)$. See the Figure $4-5$ point 1 . Since there is only 1 point, no vector is created.

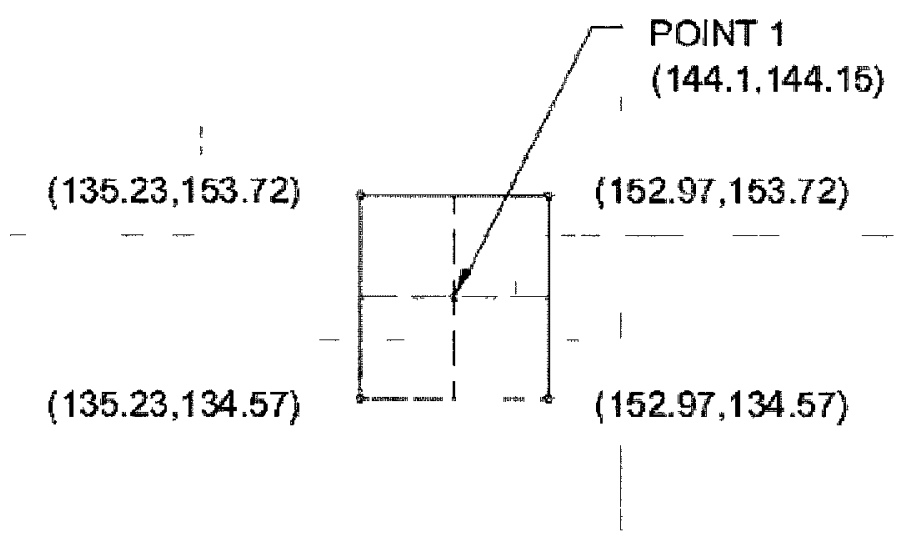

Figure 4-5: The first location point BS generated: Point 1

2. After the $1^{\text {st }}$ round, BS cleans up the data and starts the $2^{\text {nd }}$ round collection. In the $2^{\text {nd }} 30$ seconds collection period, BS receives two points. See Table 4-3. BS generates the $2^{\text {nd }}$ point and the first vector $\vec{p}_{1}$ with the direction from point 1 towards point 2 using Equation 4-5: $\left(g x_{1}, g y_{1}\right)=(136.98,149.60)$. The angle $\alpha_{1}$ is calculated using the Equation4-6: $\alpha_{1}=a \tan \left(\frac{g y_{1}-g y_{0}}{g x_{1}-g x_{0}}\right)=143.18^{\circ}$. See the points in the Figure 4-6. In the $2^{\text {nd }}$ round, one vector is created. 
Table 4-3: Center gravity points $B S$ received in the second $W=30$ seconds

\begin{tabular}{|c|c|}
\hline$X(2)$ & $Y(2)$ \\
\hline 13804 & 14996 \\
\hline 13587 & 149.03 \\
\hline
\end{tabular}

3. In the $3^{\text {rd }}$ round, repeat the step $3, \mathrm{BS}$ gets the $3^{\text {rd }}$ location point at coordinates $\left(g x_{2}, g y_{2}\right)=(142.57,141,85)$. The angle $\alpha_{2}$ of vector $\vec{p}_{2}$ is calculated using the Equation 4-6: $\alpha_{2}=a \tan \left(\frac{g y_{2}-g y_{1}}{g x_{2}-g x_{1}}\right)=306.28^{\circ}$. See Figure 4-7. The angle difference between $\alpha_{1}$ and $\alpha_{2}$ is $\left|\alpha_{2}-\alpha_{1}\right|=164.10^{\circ}$

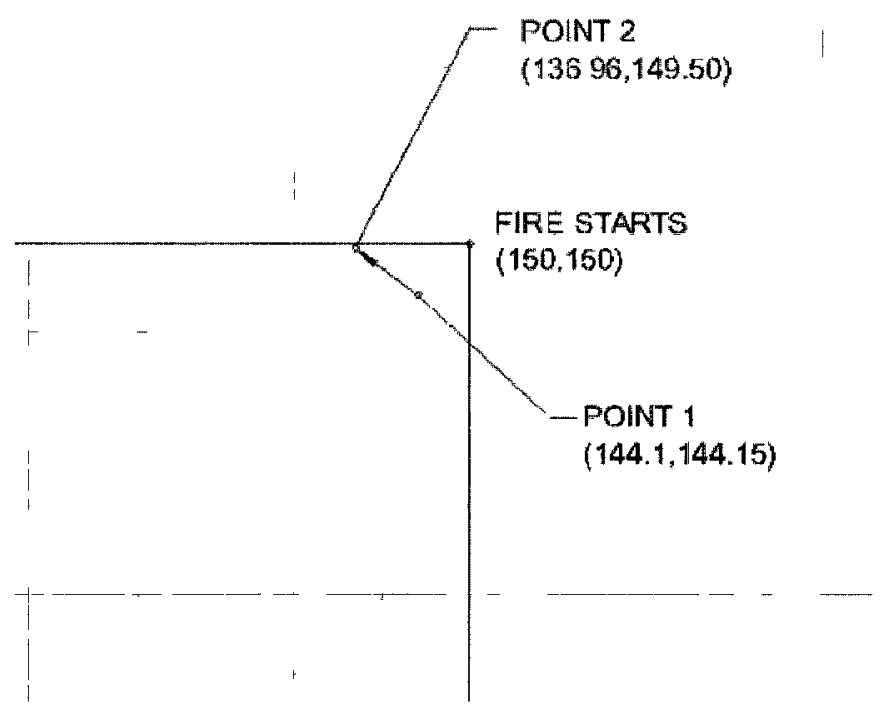

Figure 4-6: The $1^{\text {st }}$ and $2^{\text {nd }}$ location points: point 1 and point 2 , the first vector

4. By repeating the step 4 round after round the more points and angles are generated until the simulation stops. The total number of points is 11 and the 
number of vectors is 10 . See the Figure 4-8. The summary of the results is in the table 4-4.

Globally, the center of gravity points are moving within the propagation area (shaded area). Most of the vectors are oriented in the fire spreading direction - SouthWest direction, while a few of them are off the South-West direction. According to the protocol, the center of gravity points is calculated based on the missing neighbor area, the sensor distribution density plays a very important role. The fewer nodes are in the area, the less data is collected, and the more inaccurate the estimation is.

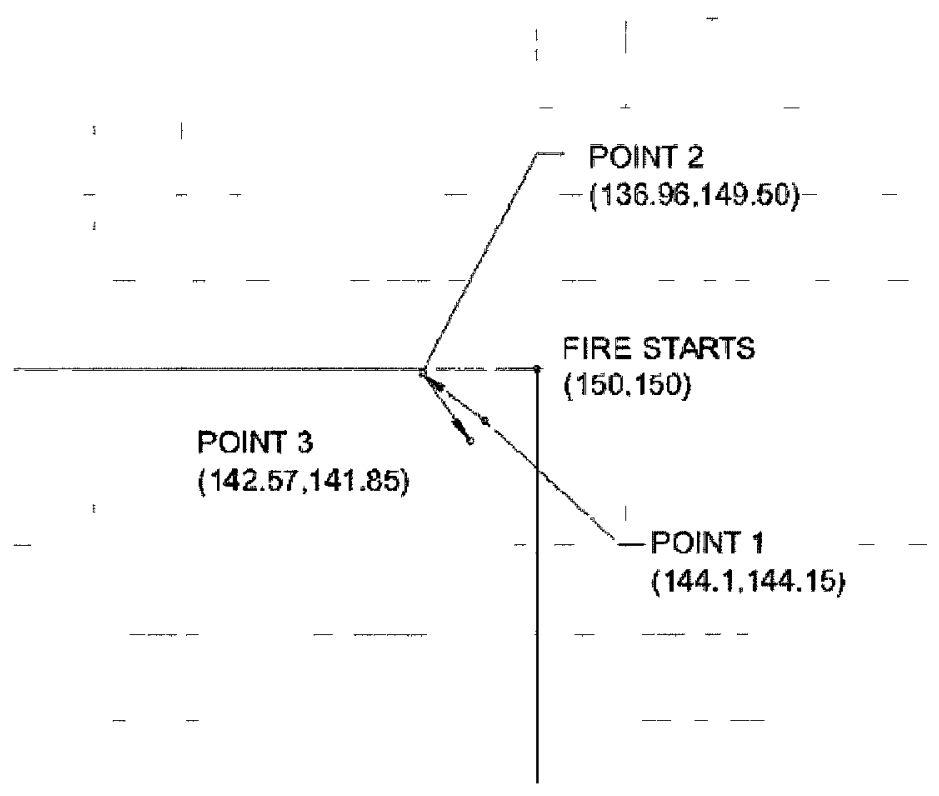

Figure 4-7: 3 location points and 2 vectors

Based on the data in table $4-4$, the last 2 vectors $\vec{p}_{9}$ and $\vec{p}_{10}$ has the direction angle $\alpha_{9}=208.2^{\circ}$ and $\alpha_{10}=205.96^{\circ}$. The difference between $\alpha_{9}$ and $\alpha_{10}$ is $2.24^{\circ}$ which is less than our defined threshold $15^{\circ}$. Thus, using simple estimation algorithm, we conclude that the fire is spreading to the direction of $\vec{p}_{10}$, with the angle of $205.96^{\circ}$. 


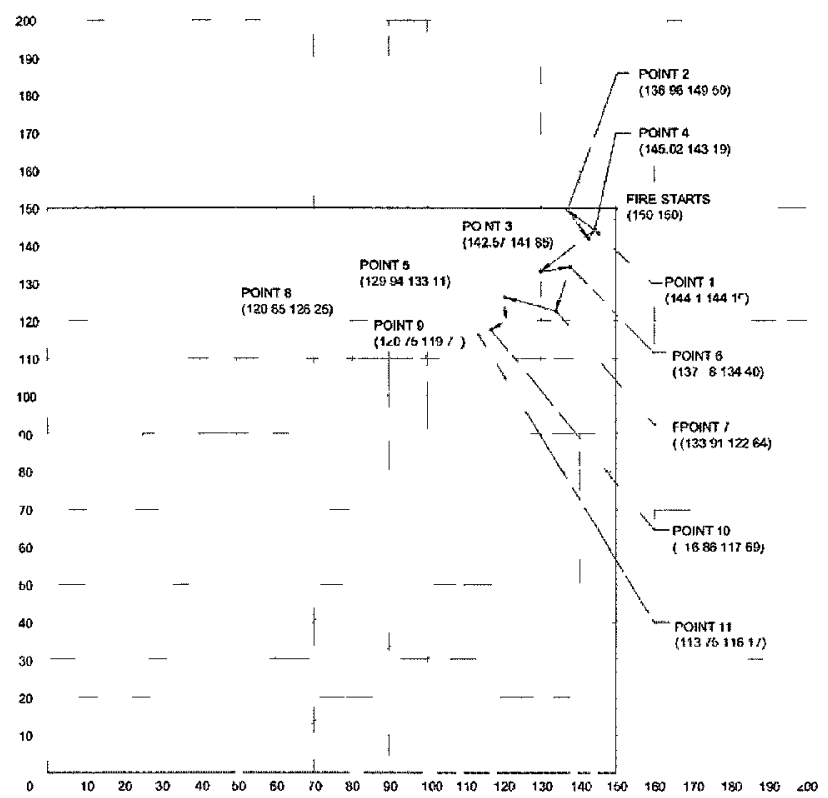

Figure 4-8: BS generates 11 points and 10 vectors

Table 4-4: The summary of the results

\begin{tabular}{|c|c|c|c|c|c|}
\hline Pounts $(j)$ & $g x_{3}$ & $g y_{3}$ & Vector & Angle $\alpha_{3}()^{\prime}$ & $\mid \sigma_{3} \alpha_{3-1} I()$ \\
\hline 0 & 1441 & 14415 & & & \\
\hline 1 & 13696 & 14950 & $\vec{p}_{1}$ & 14318 & \\
\hline 2 & 14257 & 14185 & $\vec{p}_{2}$ & 30628 & 16410 \\
\hline 3 & 14502 & 14319 & $\vec{p}_{3}$ & 2853 & 27775 \\
\hline 4 & 12994 & 13311 & $\vec{p}_{4}$ & 21374 & 18521 \\
\hline 5 & 13778 & 13440 & $\vec{p}_{5}$ & 935 & 20439 \\
\hline 6 & 13391 & 12264 & $\vec{p}_{6}$ & 25179 & 24244 \\
\hline 7 & 12065 & 12625 & $\vec{p}_{7}$ & 16476 & 8703 \\
\hline 8 & 12075 & 11977 & $\vec{p}_{8}$ & 27085 & 10609 \\
\hline 9 & 11686 & 11769 & $\vec{p}_{9}$ & 2082 & 6265 \\
\hline 10 & 11375 & 11617 & $\vec{p}_{10}$ & 20596 & 224 \\
\hline
\end{tabular}

According to statistical algorithm, we have classified the angles collected in table 4-4 into the 4 sections, and calculated the percentage of angles in each section in table 45. The section 3 has $40 \%$ of angle collected. Therefore BS reaches the weak conclusion that the fire is spreading towards section3 South-West direction. 
Table 4-5: Number of vectors and the percentages in each section

\begin{tabular}{|c|c|c|c|c|}
\hline & $\begin{array}{c}\text { Section 1 } \\
0-90\end{array}$ & $\begin{array}{c}\text { Section 2 } \\
90-180\end{array}$ & $\begin{array}{c}\text { Section 3 } \\
180-270\end{array}$ & $\begin{array}{c}\text { Section 4 } \\
270-360\end{array}$ \\
\hline Number of Vectors & 2 & 2 & 4 & 2 \\
\hline Percentage & $20.00 \%$ & $20.00 \%$ & $40.00 \%$ & $20.00 \%$ \\
\hline
\end{tabular}

\subsubsection{Simulation Results}

To validate the algorithm, we propose three scenarios. Scenario 1 is that the fire starts at $(100,0)$ and spreads to North with the angle $60^{\circ}$. Scenario 2 is that the fire starts at $(50,50)$ and spreads to Northeast direction with angle $90^{\circ}$. These two cases can be treated as the situations where obstacles are restricting the propagation of the fire or the case that strong wind is applied. Scenario 3 is that the fire starts at the center $(100,100)$ and spreads evenly in all direction that could be the open space with the similar type of fuels and no obstacles and no wind situation, or in the big warehouse with the same material, no obstacles and no wind.

These 3 scenarios are depicted in the Figure 4-9; Fire is propagating in the shadowed area. We perform tests using both simple algorithm and statistical algorithm.

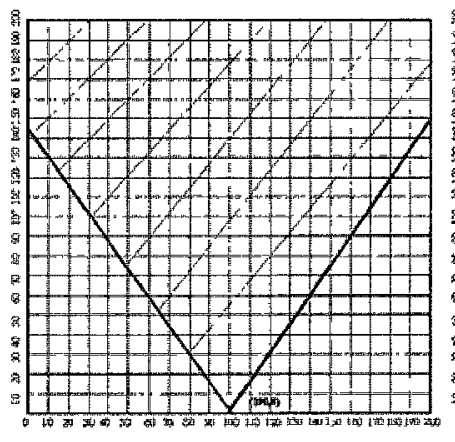

a. scenario 1

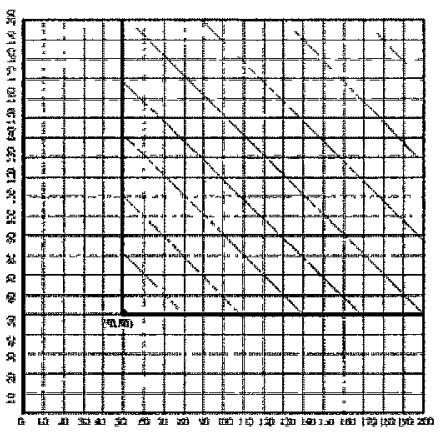

b. scenario 2

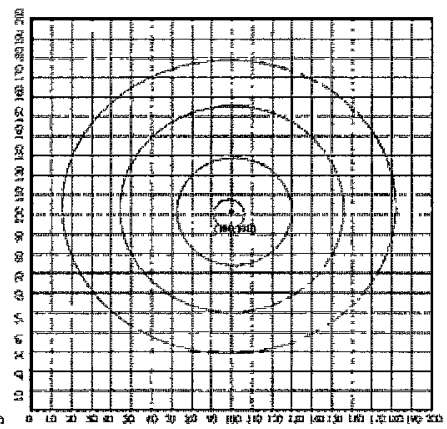

c. scenario 3

Figure 4-9: Fire propagation scenarios 


\section{A. Comparison of the simple algorithm and statistic algorithm}

\section{A-1: simple algorithm}

The angle threshold for simple estimation algorithm is defined $\alpha_{t h}=15^{\circ}$ which means when the angle difference between two executive vectors is less than $15^{\circ}$, BS will conclude that the fire spreading direction is the direction of the last vector. Based on the simple algorithm, the results of these three scenarios with simple algorithm are in the table 4-6. The final direction angle BS determined is displayed in the table.

For Scenario 1 , the fire spreads towards North with $60^{\circ}$. Obviously, from the Figure 4-9, the spreading area of the fire falls in the angle from $60^{\circ}$ to $120^{\circ}$ toward North. From table 4-6, even though all ten tests are reached conclusions, some of the results predict the direction out of the real fire propagation range which will be considered failure. So the success rate is $60 \%$.

Table 4-6: The results of the 3 scenarios with simple algorithm

\begin{tabular}{l}
\multicolumn{2}{c|}{ Scenario 1 } & \multicolumn{2}{c|}{ Scenario 2 } & \multicolumn{2}{c|}{ Scenario 3 } \\
\begin{tabular}{|c|c|c|c|c|c|}
\hline times & direction(angle) & times & direction(angle) & times & direction(angle) \\
\hline 1 & 78.56 & 1 & 1.21 & 1 & 37.77 \\
\hline 2 & 8759 & 2 & 163.23 & 2 & 2544 \\
\hline 3 & 132.52 & 3 & 92.79 & 3 & 4506 \\
\hline 4 & 3067 & 4 & 45.7 & 4 & 25452 \\
\hline 5 & 133.86 & 5 & 64.01 & 5 & 89.95 \\
\hline 6 & 36.22 & 6 & 51.94 & 6 & no conclusion \\
\hline 7 & 103.93 & 7 & 119.02 & 7 & no conclusion \\
\hline 8 & 77.25 & 8 & no conclusion & 8 & no conclusion \\
\hline 9 & 114.08 & 9 & no conclusion & 9 & 127.21 \\
\hline 10 & 83.05 & 10 & 38.21 & 10 & no conclusion \\
\hline
\end{tabular}
\end{tabular}

For Scenario 2, 20\% of test could not reach the conclusion, and $30 \%$ of test predicted the direction out of the fire propagation range which is $0^{\circ}-90^{\circ}$, for instance 
$119.02^{\circ}$. Therefore, the successful rate is $50 \%$. For scenario 3 , fire spreads in all direction. Theoretically, this algorithm is not applicable to this case. However, the results shows $60 \%$ of time BS reaches conclusion with the certain direction that cannot correctly describe the propagation of the fire; "no conclusion", on the other hand, is closer to the real situation.

\section{A-2: Statistical algorithm}

When applying statistical algorithm with the four global sections defined, BS determines which section the fire is spreading to instead of the exact direction. We define the maximum vectors BS collected is 11 then BS will make the decision based on the collections. The maximum number of vectors limits the data the decision based on and the time BS takes to make the decision. The larger number is, the long time it will take since each round creates one vector, but the more accurate the decision will be. It is possible that BS could not collect enough vectors during the simulation period due to the sensor density in the area or the no sensor to relay the message to the BS. In that case, BS will have to depend on what it has to decide the direction of spread. The results are displayed in the table 4-7.

Table 4-7: The results of 3 scenarios with static algorithm

\begin{tabular}{|c|c|c|c|c|c|c|c|c|c|c|}
\hline & & & & & & & & & & \\
\hline times & 1 & 2 & $\underline{3}$ & 4 & 5 & 6 & 7 & 8 & 9 & 10 \\
\hline section predicted & $1^{*}+2$ & 1 & 1 & 1 & $1^{*}+2$ & 1 & $1^{*}+2^{*}$ & $1+2^{*}$ & $2^{*}$ & 2 \\
\hline & \multicolumn{10}{|c|}{ Scenario 2 Fire started at $(50,50)$ spreads to North-East with 90 degree angle } \\
\hline times & 1 & 2 & 3 & 4 & 5 & 6 & 7 & 8 & 9 & 10 \\
\hline section predicted & 1 & 1 & 1 & 1 & $1^{*}$ & 1 & all direction & 1 & 1 & $1^{*}$ \\
\hline & \multicolumn{10}{|c|}{ Scenario 3 Fire starts at $(100,100)$ and spreads in circle } \\
\hline tumes & 1 & 2 & 3 & 4 & 5 & 6 & 7 & 8 & 9 & 10 \\
\hline section predicted & 1 & all directian & all direction & all directian & all direction & $1^{*}$ & ail direction & all direction & $1^{*}$ & $1^{*}$ \\
\hline
\end{tabular}


Table 4-7 shows the results of the 3 scenarios with statistical algorithm. $1^{*}$ means BS determines fire is towards section 1 with weak conclusion, 1 means in section 1 with the firm conclusion. $1^{*+2 *}$ means both section 1 and section 2 with week conclusion.

Fire in the Scenario 1 is spreading to the part of the 2 sections, therefore $40 \%$ of the tests predict the fire propagation in both section 1 and 2 while the rest $60 \%$ predicted either in section or in section 2 which are partially correct. Scenario 2 is spreading in the section 1 . The simulation predicts $90 \%$ results in the correct section. Statistical algorithm performs better than the simple algorithm for the concentric spreading scenario $3,60 \%$ of time BS concludes fire spreading in all direction.

Figure 4-10 shows the comparison of the performance of simple algorithm and statistical algorithm.

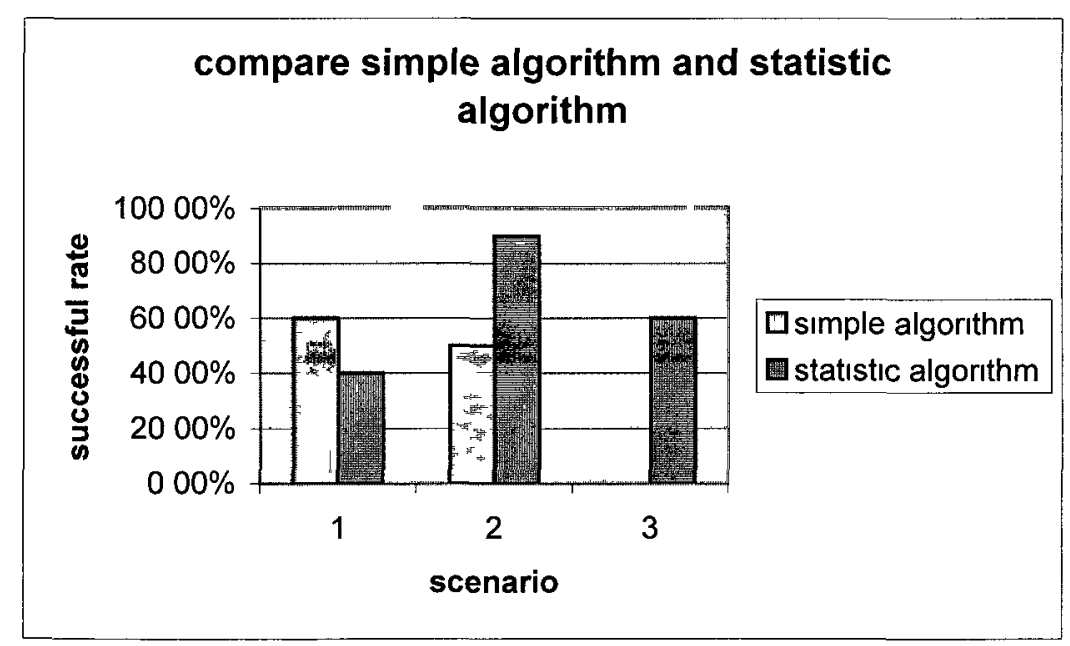

Figure 4-10: Comparison the successful rate with the different algorithm

- Simple algorithm gains high rate at the scenario 1 , but it is not applicable to the scenario 3. 
- Statistical algorithm shows advantage at scenario 2, and scenario 3, but does not have better performance at scenario 1 .

- Simple algorithm gets better results when the fire spreads with narrow direction, while statistical algorithm is more suitable to the large area propagation prediction.

\section{B. Compare the maximum number of vectors collected for statistical algorithm}

Statistical algorithm, as the name implies, the more data collected, the better results it will achieve. In this section we double total number of vectors BS collected to 22 vectors before $\mathrm{BS}$ make the decision. However in our protocol the more vectors means the longer time it takes to conclude the direction as adding one vector will add 30 seconds time. The results of the 22 vectors are in the Table 4-8.

Table 4-8: The results of 3 scenarios with statistical algorithm with maximum number of vector $=22$

\begin{tabular}{|c|c|c|c|c|c|c|c|c|c|c|}
\hline \multirow{2}{*}{ tımes } & \multicolumn{10}{|c|}{ Scenario 1 Fire starts at $(100,0)$ and spreads to above $\overline{50}$ degree angle } \\
\hline & 1 & 2 & 3 & 4 & 5 & 6 & 7 & 8 & 9 & 10 \\
\hline section predicted & $1^{*}+2^{*}$ & $1=$ & 2 & $2^{*}$ & $1^{*}$ & $1+2^{*}$ & 2 & 1 & $1+2^{*}$ & $1^{*}+2^{*}$ \\
\hline & \multicolumn{10}{|c|}{ Scenaro 2 Fire started at $(50,50)$ spreads to North-East with 90 degree angle } \\
\hline tımes & 1 & 2 & 3 & 4 & 5 & 6 & 7 & 8 & 9 & 10 \\
\hline \multirow[t]{2}{*}{ section predicted } & 1 & 1 & 1 & $1^{*}$ & 1 & 1 & 1 & 1 & 1 & 1 \\
\hline & \multicolumn{10}{|c|}{ Scenario 3 Fire starts at $(100,100)$ and spreads in circle } \\
\hline times & 1 & 2 & 3 & 4 & 5 & 6 & 7 & 8 & 9 & 10 \\
\hline \multirow[t]{2}{*}{ section predicted } & 1 & all direction & all direction & all directıon & $1^{ \pm}+3^{*}$ & 1 & 1 & all direction & all direction & all direction \\
\hline & \multicolumn{10}{|c|}{ Scenario 3-Reylsed Fire starts at $(100,100)$ and spreads in circle } \\
\hline tımes & 1 & 2 & 3 & 4 & 5 & 6 & 7 & 8 & 9 & 10 \\
\hline section predicted & all direction & all direction & all direction & all direction & 1 & all direction & all directıon & all direction & Fall & all direction \\
\hline
\end{tabular}

Table 4-8 shows the results of the 3 scenarios with statistical algorithm maximum number of vector $=22.1 *$ means fire is spreading to section 1 with weak conclusion. 1 
means to section 1 with the firm conclusion. " $1 *+2 *$ " means both section 1 and section 2 with week conclusion.

Scenario 1 has the estimation in both section 1 and section 2 with $40 \%$ successful rate, but still has $60 \%$ of results in either section 1 or 2 . Scenario 2 estimates $100 \%$ successful rate of section 1 , while scenario 3 has $60 \%$ successful rate. The figure $4-11$ demonstrates the comparison of the 11 vectors (table 4-7) and 22 vectors (table 4-8).

Increasing the maximum number of vectors improves the estimation result of scenario 2 to $100 \%$ successful rate. Even though the results for scenario 1 and 3 do not increase, they keep the same rate. Scenario 1 has the same results for both 11 vectors and 22 vectors. It implies either the statistic algorithm may not be able to represent the scenario that covers more sections or the definition of the global section needs to be adjusted to include more sections, for instance eight sections instead of four sections.

Scenario 3 also gets the same results as 11 vectors. By analyzing the results in detail, we find out that even though the max vector is 22 , during ten simulation tests, none of them collects the 22 vectors. Most of them collects 9-13 vectors, with the last point is very close to BS. It could because either the fire spreading fast or the ping cycle is too long that sensors did not have time to detect the fire situation before it is burnt, and/or BS processes the location message less often. When the fire is spread towards the edge of the grid there are no sensors available outside the grid to detect and send the update to BS. This is once again proved the algorithms are subject to distribution of the node. By decreasing the fire spreading rate in half and by making sure BS collects 22 vectors, Scenario 3 improves the results by $20 \%$. See Figure $4-9$. 


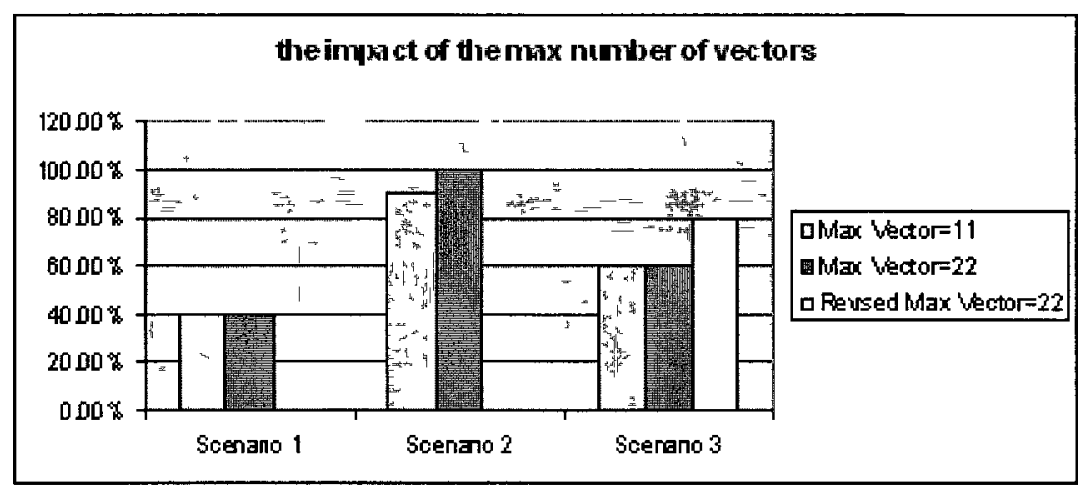

Figure 4-11: Comparison of the successful rate with the different maximum number of vector collected using statistic algorithm

For the tests presented in this section, we can derive the following conclusions:

- Statistical algorithm gets the better results by increasing maximum vectors BS collected to make decision. However, it trades off the longer time BS takes to reach the conclusion because the processing period is fixed.

- Scenario 1 implies how to define the sections is very important to statistical algorithm. It may get better result with eight sections defined instead of four sections.

- BS processing period and sensor ping cycle need to be optimized based on the different fire cases.

\section{Impact of the sensor density}

BS receives data from sensors, and based on the data to estimate the direction. The data is collected by sensors and transmitted to BS by sensor network. The accuracy of the fire propagation estimation is closely related to the density of sensor nodes. 
In this section, the sensor nodes are designed to distribute in a special pattern instead of evenly distributing in the whole grid in order to demonstrate the impact of the density to the predicted results. The three distribution models are introduced in Figure 412. Distribution model 1 , sensors are distributed in the $450 \times 50$ squares positioned diagonally throughout the area. Distribution model 2 sensors are distributed in $850 \times 50$ squares symmetrically positioned. All the other areas out of the shadowed small squares do not contain sensor nodes. Distribution model 3 is the regular model used in the previous simulation testing which has 2000 sensors randomly distributed in the $200 \times 200$ gird. Sensors in the small area are evenly distributed with the same density as previous model. We also define two fire spreading model in Figure 4-13. Spreading mode 1, fire starts at center point $(100,100)$ and spreads to North with $90^{\circ}$ angle. Spreading mode 2 , fire starts at center point $(100,100)$ as well, but spreads to the South-West direction. The reason we pick $(100,100)$ as the fire starting point is to compare the impact of the sensor density around the fire starting point. The distribution 1 and 2 have different density at the $50 \times 50$ area centered by point $(100,100)$.

The estimation algorithm uses in this section is simple algorithm. Each fire spreading model is tested with two irregular distribution models respectively, and compares with the distribution model 3 which is the regular mode and has all sensors distributed randomly all over the grid. The average sensor density of distribution model 1 in the $50 \times 50$ area around the fire starting point $(100,100)$ is half of that of mode 2,3 . Model 2 and model 3 have the same sensor density near the fire starting point, but different density on the path to the BS. The overall average sensor density in the whole 
grid is increased from model 1 to 3 . The table $4-9$ shows the testing results, Figure 4-13 depicts the diagram.

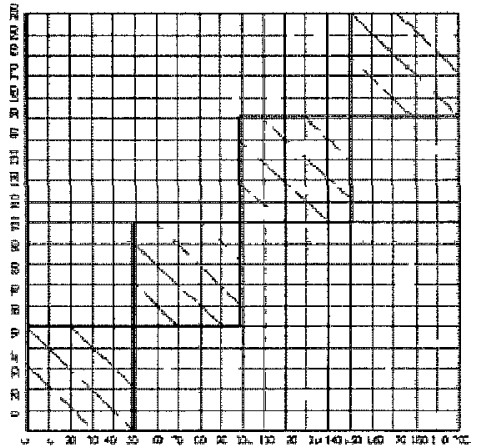

(a) $D_{1}$ tribution 1

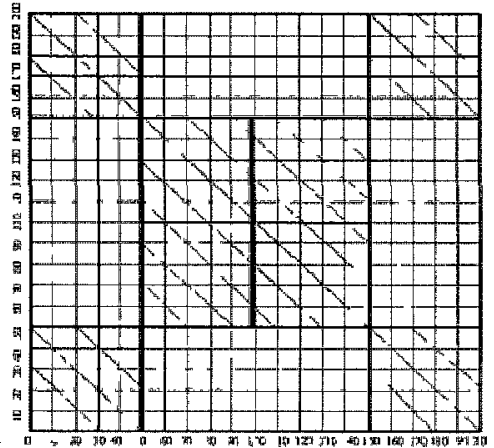

(b) Distribution 2

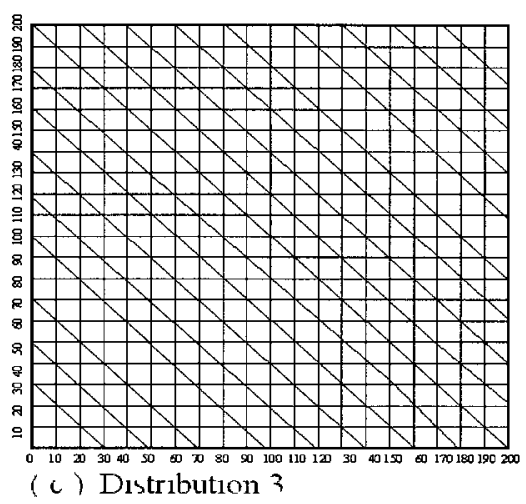

Figure 4-12: 2 irregular sensor distribution models and 1 regular distribution model

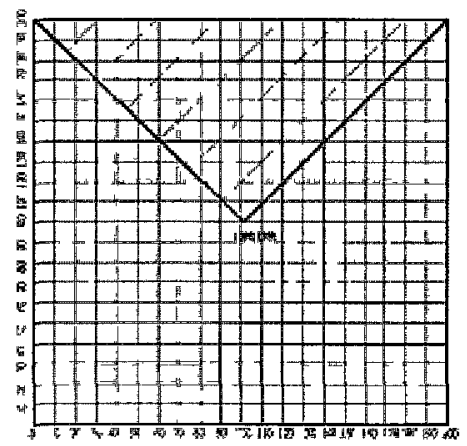

(a) Fire spresding nodel 1

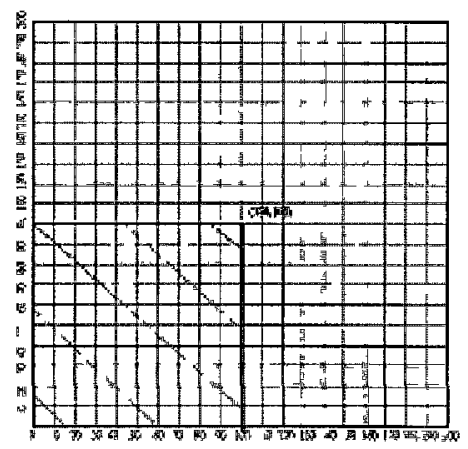

(b) Fine spreadure model 2

Figure 4-13: 2 fire spreading models 
Table 4-9: Simulation results respects to the sensor density

\begin{tabular}{|c|c|c|c|c|c|}
$\begin{array}{c}\text { fire model/ } \\
\text { distribution model }\end{array}$ & $\begin{array}{c}\text { density(sensor/cell) } \\
\text { at fire start point }\end{array}$ & $\begin{array}{c}\text { density(sensor/cell) } \\
\text { all over the grid }\end{array}$ & success & failure & N/A \\
\hline Fire 1/distribute 1 & 0.0271 & 0.0125 & $40.00 \%$ & $40.00 \%$ & $20.00 \%$ \\
\hline Fire 1/distribute 2 & 0.0542 & 0.025 & $30.00 \%$ & $60.00 \%$ & $10.00 \%$ \\
\hline Fire 1/distribute 3 & 0.054 & 0.05 & $70.00 \%$ & $30.00 \%$ & $0.00 \%$ \\
\hline & & & & & \\
\hline Fire 2/distribute 1 & 0.0273 & 0.0125 & $0.00 \%$ & $0.00 \%$ & $100.00 \%$ \\
\hline Fire 2/distribute 2 & 0.0553 & 0.025 & $0.00 \%$ & $50.00 \%$ & $50.00 \%$ \\
\hline Fire 2/distribute 3 & 0.0583 & 0.05 & $20.00 \%$ & $80.00 \%$ & $0.00 \%$ \\
\hline
\end{tabular}

In the table 4-9, "success" means that the direction BS has estimated matches the model. "Failure" means that the direction BS has estimated does not match the model. "N/A" means that the BS did not receive enough data to predict the direction, for instance, BS only receives 2 center of gravity points which only can create one vector, or BS receives less than 2 center of gravity points which no vector can be created. Thus no direction is determined.

Figure 4-14 represents the statistics corresponding to the three distribution models combined with two fire spreading models. From the distribution model 1, 2, 3 the overall sensor density is increased from left to right, the "success" is also increased, "N/A" is decreased accordingly for both fire model 1 and 2 . The exception of fire mode $1 /$ distribution 2 , success rate does not increase, but the "N/A" portion decreases which means BS receives more data when the increasing of the sensor density, but it is still not enough to predict the direction successfully. In Fire mode 2 /distribution 1 , fire is spreading towards Southwest. While temperature is increasing, the nodes close to the center area start to be burnt first. Even though some nodes at the bottom left corner generates location messages, they are not able to pass the information to BS. So the BS can not make the decision due to the lack of information. Distribution 1 has results of 
$100 \%$ NA and $0 \%$ success and failure. Along with the increasing sensor density, Fire mode 2 gets better results in distribution 2 and 3.

This set of tests proves the sensor density and the distribution pattern at the fire starting point and on the relay path to BS do impact the BS to receive the data and furthermore impact BS to estimate the disaster's propagation direction.

\begin{tabular}{|c|c|c|c|}
\hline & Distribution model 1 & Distribution model 2 & Distribution model 3 \\
\hline 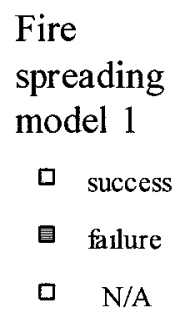 & 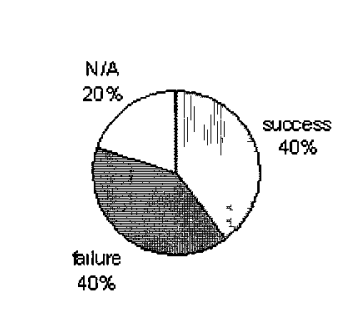 & & \\
\hline $\begin{array}{l}\text { Fire } \\
\text { spreading } \\
\text { mode } 2 \\
\square \quad \text { success } \\
0 \text { fallure } \\
\square \quad \text { N/A }\end{array}$ & 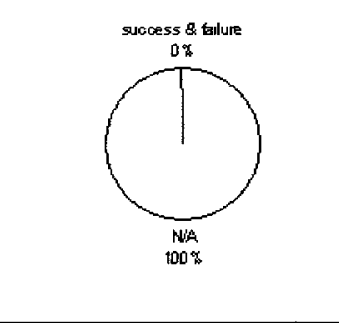 & $\begin{array}{l}\mathrm{N} / 2 \\
500\end{array}$ & \\
\hline
\end{tabular}

Figure 4-14: Comparison of impact of sensor density

\subsection{Summary}

In this chapter, we present an innovated protocol to determine the fire spreading direction after the fire alarm is received. The proposed method relies on the mutual detection of neighbors' status implemented by each sensor node. Two algorithms are proposed to help BS to make the decision. The algorithms are validated by the simulation tests. 
- Simple algorithm works better in certain direction propagation, while statistical algorithm is suitable for large area propagation. Especially the concentric propagation scenario.

- In order to get better prediction, parameters need to be optimized for both simple algorithm and statistical algorithm.

- Statistical algorithm is related to the number of vectors BS collected. The more vectors are, the better results are estimated, but the longer time it takes.

- The results are proved to be subject to the density of the sensor nodes, which are the key point of the wireless sensor network. 


\section{Chapter 5 - Conclusion}

In this thesis, we propose a novel approach of the fire detection system combining the extended temperature approach and Fuzzy Logic neighborhood approach with the simple temperature sensor, followed by the fire trajectory estimation protocol to determine the propagation of the disaster. In the following paragraphs we present what we have accomplished and what needs to be address for the future research.

Many researches have been working on the best combination of the sensor nodes for the fire detection. Recently the advanced sensor technologies allow the sensor to perform data fusion at node level. Quite a few studies apply intelligent system like the Fuzzy Logic System, FFNN to assist the sensor network for the event detection. By using the multiple sensors and enhanced with data fusion system make the fire detection system more and more robust. But all the researches simply inherit the combination of the sensors and only focus on the new data fusion technologies. Our proposed detection algorithm is using the simple temperature sensor with the strong two-level data processing: enhanced temperature checking and neighborhood agreement. Validated by both fire and nuisance test cases the simulation results demonstrate our algorithm does very good performance to report fire alarm and immunize the noise and also achieves the results that multiple sensors reached. The algorithm proves that frequently sampling the 
environment data may not report the fire alarm faster, which is very import to the energylimited wireless sensors to save the resources.

In the wireless sensor network, Base Station or control center is usually located remotely. In order to master the disaster developing status, BS needs to know the propagation trends of fire. We propose simple estimation algorithm and statistical estimation algorithm using the sensor network. Both algorithms are based on sensors' mutual status checking to discover the area that is exposed to the fire. By receiving the series of the center gravity points of the affected area, BS will have the big picture of the direction fire spreads. Both algorithms are proved to be able to estimate the fire spreading direction. However, the accuracy of the estimation is highly depended on the sensor density and the distribution.

Even though we test the protocols with fire alarm, it is applicable to the other disaster detection and prediction. Nevertheless, both the event detection algorithm and the trajectory estimation still need lots of work to improve. The following are some aspects that can be extended to improve the performance of the proposed algorithms.

- For the fire detection algorithm, we conclude that the frequently sampling the environment data might not report the fire alarm quickly. The sample period less than 5 seconds are not recommended. While the sampling periods over 15 seconds are not sensitive to the small temperature change, which may miss some important information. Also the temperature thresholds are closely related to the sampling period. Both parameters need to work together to 
optimize the best values and the best combinations to achieve the best performance.

- Adding the Fuzzy Logic to the fire detection system improves immunization of the false alarm, however it increases the fire alarm time. The Fuzzy Logic membership and the parameters may be adjusted and tested to get the better results.

- For simple estimation algorithm, angle threshold $\alpha_{t h}$ needs to be tested with the different values other than $15^{\circ}$ to find the best threshold, or the best range of the thresholds.

- For statistical estimation algorithm, number of the vectors collected impacts the accuracy of the prediction. The more vectors are, the more accurate the result is, but the longer time it takes to process. To find the optimized number of vectors, which could accurately predict the direction in the affordable period of time, is important.

- The sensor pinging period, BS processing time period and fire spreading model need to be cooperated and optimized according to the real fire situations.

- In statistical estimation algorithm, we define four sections. However it is worth to investigate the different ways to define sections. 
- We prove that sensor density in the field impacts the direction prediction. Further studies need to discover the relationship between the sensor density and the protocol performance.

- Our proposed algorithms are validated with several fire cases and nuisance cases. Both fire detection algorithms and estimation propagation algorithms may be tested with the wide varieties of fire and nuisance sources.

- Our simulation assumes the sensor knows its location. In the real world, the sensors often do not know the exact location during the development. Integrating the location algorithm into the event detection system and event propagation estimation system make the whole system more practical and realistic. 


\section{References}

1. Lloret, J.; Garcia, M.; Bri, D.; Sendra, S., A Wireless Sensor Network Deployment for Rural and Forest Fire Detection and Verification. Sensors 2009, 9, 8722-8747.

2. Bahrepour, M.; Meratnia, N.; and Havinga, P.J.M., Automatic Fire Detection: A Survey from Wireless Sensor Network Perspective. 2008, Centre for Telematics and Information Technology, University of Twente, Enschede, Netherlands.

3. Barnes, M.; Leather, H.; and Arvind, D.K., "Emergency Evacuation using Wireless Sensor Networks," Local Computer Networks, 2007. LCN 2007. 32nd IEEE Conference on, 851-857.

4. Lewis, F.L., Wireless Sensor Networks, Smart Environments: Technologies, Protocols, and Applications, ed. D.J. Cook and S.K. Das, John Wiley, New York, 2004.

5. Le, T.N.; Chong, P.H.J.; Li, Xue Jun; Leong, Wai Yie, (2010), A Simple GridBased Localization Technique in Wireless Sensor Networks for Forest Fire Detection, Communication Software and Networks, 2010. ICCSN '10. Second International Conference on, 93-98.

6. Hellmann Martin (2001, March). Fuzzy Logic Introduction. [Online] http://staff.um.edu.mt/mmon1/lectures/bit5201/FLintro.pdf

7. http://home.howstuffworks.com/home-improvement/householdsafety/fire/smoke.htm accessed in October 2010.

8. Bahrepour, M.; and Meratnia, N.; Havinga, P.J.M.; (2009), Use of AI Techniques for Residential Fire Detection in Wireless Sensor Networks. In: AIAI 2009 Workshop Proceedings, 23-25 April 2009, Greece.

9. http://en.wikipedia.org/wiki/Wireless_sensor_network, accessed in October 2010. 
10. Lu, Zhiping; Qin, Huibin; Hu, Ji; and Huang, Sufang, The Design of Wireless Sensor Networks for Forest Fire Monitoring System. 2006, White Paper: School of Electronics and Information, Hangzhou Dianzi University.

11. Lim, Yeon-sup; Lim, Sangsoon; Choi , Jaehyuk; Cho, Seongho; Kim, Chongkwon; Lee, Yong-Woo, "A Fire Detection and Rescue Support Framework with Wireless Sensor Networks," Convergence Information Technology, 2007. International Conference on, 135-138.

12. Antoine-Santoni, T.; Santucci, J. -f.; Gentili, E. De; Silvani, X.; Morandini, F., Performance of a protected wireless sensor network in a fire. Analysis of fire spread and data transmission. Sensors 2009, 9, 5878-5893.

13. Yu, Liyang; Wang, Neng; Meng, Xiaoqiao, Real-time forest fire detection with wireless sensor networks, Wireless Communications, Networking and Mobile Computing, 2005. Proceedings. 2005 International Conference on, 1214-1217.

14. Marin-Perianu, Mihai; Havinga, Paul, D-FLER A distributed Fuzzy Logic Engine for Rule-Based Wireless Sensor Networks, Proceedings of the 4th international conference on Ubiquitous computing systems, Tokyo, Japan SECTION: Sensing systems and sensor network, Pages: 86-101, 2007.

15. Mendel, J.M., Fuzzy logic systems for engineering: a tutorial, Proceedings of the IEEE , vol.83, no.3, pp.345-377, Mar 1995 doi: 10.1109/5.364485.

16. http://en.wikipedia.org/wiki/Feedforward neural network, accessed in October 2010.

17. Haykin, Simon, FEEDFORWARD NEURAL NETWORKS: AN INTRODUCTION.

18. http://www.learnartificialneuralnetworks.com/, accessed in October 2010.

19. Liang, Q.; Wang, L., Event detection in wireless sensor networks using fuzzy logic system, Computational Intelligence for Homeland Security and Personal 
Safety, 2005. CIHSPS 2005. Proceedings of the 2005 IEEE International Conference on, vol., no., pp.52-55, March 31 2005-April 12005.

20. Kapitanova, Krasimira; Son, Sang H.; and Kang, Kyoung-Don, Event Detection in Wireless Sensor Networks - Can Fuzzy Values be Accurate?, 2nd International Conference on Ad Hoc Networks (AdHocNets) 2010, Victoria, Canada, August 2010.

21. Bahrepour, M.; Meratnia, N.; Havinga, P.J.M.; , Sensor fusion-based event detection in Wireless Sensor Networks, Mobile and Ubiquitous Systems: Networking \& Services, MobiQuitous, 2009. MobiQuitous '09. 6th Annual International, vol., no., pp.1-8, 13-16 July 2009.

22. Akyildiz, I.F.; Su, W.; Sankarasubramaniam, Y.; and Cayirci, E., Wireless sensor networks: a survey, Computer Networks, vol. 38 no. 4, pp. 393-422, March, 2002.

23. Baggio, A. and Langendoen, K., Monte-Carlo localization for mobile wireless sensor networks, in Second International Conference on Mobile Ad-hoc and Sensor Networks (MSN 2006).

24. Osterlind, F.; Pramsten, E.; Roberthson, D.; Eriksson, J.; Finne, N.; Voigt, T., Integrating building automation systems and wireless sensor networks, Emerging Technologies and Factory Automation, 2007. ETFA. IEEE Conference on , vol., no., pp.1376-1379, 25-28 Sept. 2007.

25. Tubaishat, M. and Madria, S., Sensor Networks: An Overview, IEEE Potentials, 2003, 22(2):20-23.

26. He, T.; Krishnamurthy, S.; Stankovic, J.; Abdelzaher, T.; Luo, L.; Yan, T.; Stoleru, R.; Gu, L.; Zhou, G.; Hui, J. and Krogh, B., VigilNet: An Integrated Sensor Network System for Energy Efficient Surveillance, ACM Transactions on Sensor Networks, to appear. 
27. Virone, G.; Wood, A.; Selavo, L.; Cao, Q.; Fang, L.; Doan, T.; He, Z.; Stoleru, R.; Lin, S. and Stankovic, J., An Assisted Living Oriented Information System Based on a Residential Wireless Sensor Network, Proceedings D2H2, May 2006.

28. Szewczyk, R.; Osterweil, E.; Polaster, J.; Hamilton, M.; Mainwaring, A.; and Estrin, D., Habitat monitoring with sensor networks. Communications of ACM 47,6 (2004), 34-40.

29. Glaser, S. D., Some real-world applications of wireless sensor nodes. In Proceedings of SPIE Symposium on Smart Structures and Materials/ NDE (San Diego, CA, USA, Mar.2004).

30. Shen, Xingfa; Wang, Zhi; Sun, Youxian, Wireless sensor networks for industrial applications, Intelligent Control and Automation, 2004. WCICA 2004. Fifth World Congress on, vol.4, no., pp. 3636-3640 Vol.4, 15-19 June 2004.

31. http://www.nifc.gov/fire info/nfn.htm, accessed in October 2010.

32. http://www.nfpa.org/assets/files/PDF/OS.fireloss.pdf, accessed in October 2010.

33. Bryan, J. L. (1988). Behavioral Response to Fire and Smoke, SFPE Handbook of Fire Protection Engineering,, National Fite Protection Association, Quincy.

34. Shurmer, H. V. and Gardner, J. W. (1992). Odour Discrimination with an Electronic Nose. Sensors andActuators B: 1-11.

35. Milke, J. A. and McAvoy, T. J. (1995). Analysis of signature patterns for discriminating fire detection with multiple sensors. Fire Technology 31(2): 120136.

36. Milke, J. A. (1999). Using Multiple Sensors for Discriminating Fire Detection. Fire Suppression and Detection Research Application Symposium, National Fire Protection Research Foundation. 
37. Gottuk, D. T.; Peatross, M. J., et al. (2002). Advanced fire detection using multisignature alarm algorithms. Fire Safety Journal 37(4): 381-394.

38. Bahrepour, Majid ; Zwaag van der, Berend Jan ; Meratnia, Nirvana and Havinga, Paul (2010) Fire data analysis and feature reduction using computational intelligence methods. In: Second KES International Symposium on Intelligent Decision Technologies, IDT 2010, 28-30 July 2010, Baltimore, Maryland, USA.

39. Bagheri, M. (2007). Efficient K-Coverage Algorithms for Wireless Sensor Networks and Their Applications to Early Detection of Forest Fires. Computing Science, SIMON FRASER UNIVERSITY. MSc: 75.

40. Tan, W.; Wang, Q. et al. (2007). Mine Fire Detection System Based on Wireless Sensor Network. Information Acquisition, 2007. ICIA '07. International Conference on.

41. Muller, H.C.; Fischer, A., A robust fire detection algorithm for temperature and optical smoke density using fuzzy logic, Security Technology, 1995. Proceedings. Institute of Electrical and Electronics Engineers 29th Annual 1995 International Carnahan Conference on, vol., no., pp.197-204, 18-20 Oct 1995.

42. Chen, S.; Bao, H. et al. (2003). A fire detecting method based on multi-sensor data fusion. Systems, Man and Cybernetics, 2003. IEEE International Conference on.

43. Cestari, L. A.; Worrell, C. et al. (2005). Advanced Fire Detection Algorithms Using Data from the Home Smoke Detector Project. Fire Safety Journal 40: 1-28.

44. Fleming, J. and Robertson, R. G. (2003). Fire Management Tech Tips: The Osborne Fire Finder. T. R. 1311-SDTDC, USDA Forest Service.

45. Breejen, E.; Breuers, M. et al. (1998). Autonomous forest fire detection. Third International Conference on Forest Fire Research and Fourteenth Conference on Fire and Forest Meteorology, Luso, Portugal. 
46. Filippoupolitis, Avgoustinous; Hey, Laurence; Loukas, Georgios; Gelenbe, Erol; Timotheou, Stelios; Emergency Response Simulation Using Wireless Sensor Networks, Ambi-Sys '08 Proceedings of the 1st international conference on Ambient media and systems (C2008

47. http://www.wrh.noaa.gov/sew/fire/olm/nfdrs.htm, accessed in October 2010.

48. Vescoukis, V.; Olma, T. et al. (2007). Experience from a Pilot Implementation of an "In- Situ" Forest Temperature Measurement Network. Personal, Indoor and Mobile Radio Communications (PIMRC2007).

49. Bernardo, L.; Oliveira, R. et al. (2007). A Fire Monitoring Application for Scattered Wireless Sensor Networks: A peer-to-peer cross-layering approach, International Conference on Wireless Information Networks and Systems (WINSYS'07), Barcelona, Spain.

50. Tripathy,Malay Ranjan; Gaur, Kunal; Sharma, Sonam and Virdi, G. S., Energy Efficient Fuzzy Logic Based Intelligent Wireless Sensor Netowrk. Progress In Electromagnetics Research Symposium Proceedings, Cambridge, USA, July 5-8, 2010.

51. http://cs.gmu.edu/ eclab/projects/mason/ accessed in October 2010.

52. http://smokealarm.nist.gov/, accessed in October 2010.

53. http://www.isi.edu/nsnam/ns/ visited in November 2010

54. http://www.worldscibooks.com/etextbook/6288/6288 chap1.pdf visited in November 2010

55. http://www.mathworks.com/products/matlab/ visited in November 2010 